\title{
Compte rendu des travaux du Comité Technique de la Société Hydrotechnique de France
}

\author{
Meetings of the technical Committee
}

\section{Session des 19 et 20 juin 1951}

English synopsis, p. 114.

Ont participé aux travaux de cette session :

a) Conseil d'administration :

I. Garnel, Président.

Mi. Coyne, Devun, Hupner, Nizery, de Rouvilze.

b) Adhérents :

Electricité de France, représentée par MM. Bouvard, Cappus, Chabert, Dupuy, Lugiez, Maithe, Marquenet, MChon, Morlat, SchneEbeli, Vantroys.

Electricité et Gaz d'Algérie représenté par M. Crosnar.

Le Bureau d'Etudes A. Coyne et J. Bellier, représenté par M. LEgros.

Les Etablissements Neyrpic, représentés par MM. Barbe et WaLleT.

L'Entreprise Campenon-Bernard, représentée par M. Arrin.

La S.N.C.F., représentée par M. LAZARD.

La Compagnie Industrielle de Travanx, représentée par M. Chautru.

La Société Hersent, représentée par M. KHelstorsiy.

c) Comité technique :

I. Ie Président Barrillon.

M. Radiguer, Président des Commissions de Révision des Cahiers des Charges.

M. Escande, Président de la Commission de Révision du Code d'Essais des Installations Hydrauliques.

M. Bergeron, Vice-Président de la Section « Machines $»$. M. REMĔnÉras, Secrétaire général.

Mi. Banal, Bardon, Bellier, Benoist, Blanchet, Cot Pierre, Dupouy, Gaurheron, Gibert, Govgrnhem, Gri-

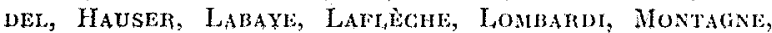

de Saint-Vaulry, Sanson, Sauvacie de Sain'i-Marc, Semra, Silber, Tissier, Vennin.

M. Boyer, Secrétairc.

II. Chabanerx, représentant M. Chamayou.

MM. Devineux et Gerlifr, représentant M. J. Laungnt.

M. MonLET, représentant M. LEscall.

M. Ronin, représentant M. Duffaut.

\section{d) Invités:}

M. Martinot-LaGarde, Directeur de l'Institut de Mécanique des Fluides de Lille.

M. Christian Beav, Président de la Société l'Energie des Mers.

M. Kiveliovirch, du Service Météorologique de Ia Métropole et de l'Afrique du Nord.

M. Merot, du Service "Technique des Grands Barrages.

M. Mesnager, Ingénieur conseil.

M. PÉpin, de la Société Industrielle de Traitement des Liquides et des Gaz.

M. Desmur. de la Société Rateau.

Se sont excusés :

Mi. Bablnet, Batiche, Coguand, Coutagne, Dheatrik, EsClangon, Gaden, GaGe, HÉnin, Ledoli, MErier, Missings de Sourbier, Parde, Schlag, Sutziée, Viberic.

\section{Adoption du procès-verbal de la session des 14,15 et 16 mars 1951}

Le procès-verbal de la session des 14,15 et 16 matrs 1951, n'ayant donné lieu à ancunce observation, est adopté. 


\section{SEAANCE DU MARDI MATIN 19 JUIN 1951}

La séance est ouverte sous la présidence de M. BanmuLon, Plésident.

\section{Nécrologie}

M. le Président fait part au Comité l'echnique du décès de :

M. Counsis, Membre du Conseil d'Administration de la S.H.F.

M. Laronde, Membre de nos sections « Essais el Mesures », et «Glaciologie » et de la Commission de Revision du Code d'Essais des Installations Hydrauliques.

M. TRomente des Commissions du Cahier des Charges des Turbines Hydranliques et du Code d'Essais des Installations Hydrauliques.

Le Comité Technique renouvelle ses condoléances à leurs familles.

\section{Distinctions honorifiques}

I. le Président exprime leś félicitations du Comité l'echnique à M. Alblenet, Vice-Président de la S.H.F. et a MM. Hupner et Peltier, Administrateurs de la S.H.F., tous trois promus Officiers de la Légion d'Honneur.

\section{Admissions}

II. le Président propose l'admission au Comité Technique de:

M. Lavarois, Ingénieur en Chef à la Direction de l'Exploitation d'Electricite de France, Service des Usines Hydrauliques.

M. Mesvager, Ingénieur Conseil hydraulicien.

Ces admissions, adoptées par le Comité ont été soumises au Conseil d'Administration qui les a ratifiées dans sa séance du 20 juin 1951.

\section{Documents reçus depuis la dernière session}

I. le Président fait part des documents ci-apres reçus par la S.H.F., depuis sa dernière session :

Don de Mme Muon. - Un certain nombre d'ouvases ayant appartenu a M. le Président Muon; précieux solvenir qui reflète l'évolution de la pensée de notre regretté Président et les questions auxquelles il portait son attention.

Don de M. Eyooux, Président honoraire du Comité Technique. - Un nombre important de manuels, lives de cotirs, plaquettes et annales qui compléteront notre bibliothèque.

Don de la Librairie Belin. - Collection de l'album en deux tomes édité à l'occasion du $\mathrm{II}^{\circ}$ Congrès de la Houille Blanche à Lyon, en septembie 1914, et contenant notamment les actes de ce Congrès; ces alloums seront distribués aux lauréats des prix S.H.F. (prix d'Hydrotechnique, prix de Monographie, etc..). - Album en deux tomes de la Chambre Syndicale des Forees Hydrauliques (1911). - Album de l'Energic Electrique du Littomal Méditerraméen et de la Société des Forces Motrices de la Haute-Durance.

En remerciant les donateurs, M. le Président adresse un appel aux Membres qui détiendraient des mémoipes ou procès-verbaux anciens de la S.H.F. n'ayant plus d'intérêt pour eux, afin qu'ils veuilent bien les mettre a la disposition du Secretariat; certains de ces documents étant épuisés, mais parfois encore demandés, les dons de nos Membres rendraient service a leurs Collègues et eviteraient ia la S.H.F. des frais de réimpression souvent disproportionnes avec le nombre des demandes.

\author{
Publications récentes offertes par les auteurs \\ ou reçues à titre d'échange
}

M. le Président annonce les rentrées ci-après : Annuate Hydrologique Autrichien, année 1948.

Annuaire Hydrologique Allemand, année 1946, pour les bassins du Danube, du Main, le bassin bavarois de l'Elbe et Ia Weser.

Annuaire Hydrologique Allemand, année 1947, pour les bassins inférieurs de l'Elbe et moyen du Rhin.

L. Escande. - Compléments d'hydraulique ( ${ }^{\circ}$ partic). Dunod, Paris.

H. Lacombe. - La Houle à la Côte (conférence no II du Centre de Recherches et d'Etudes Océanographiques.)

G. RÉMÉNiÉRAs. - Les Jaugeages au moulinet (conférence d'hydrologie du 23-1-1951 organisée par le C.N.R.S.).

Ch. Mallet et J. PacQuant. - Les Barrages en terre.

LuTschg et Loetscher. - Zum Wasserhaushalt des Schweizer Hochgebirges ( $1^{\text {ro }}$ et $2^{\mathrm{e}}$ parties). (Exploitation des réservoirs dans la Suisse montagneuse.)

Docteur FrIEDrICH (Bielefeld). - Interprétation des recherches lysinzétriques pour l'hydrologie souterraine.

Docteur Friedrich (Bielefeld). - Sur l'Evaporation du sol.

R. Grammann. - Climat et Eaux sonterraines dans le bassin allemand pendant l'année hydrologique 1949 et l'hiver 1949-1950.

LEPPIK (Hambours). - Les Cyclones dans l'embouchure de l'Elbe dans la première moitié dn XX⿳亠丷厂 siècle (2 tomes).

Pardé. Nouveautés sur le Hoang-Ho (Revue de Géographie de Lyon, vol. XXVI, $\mathrm{n}^{\circ} 1$ ); Abondance et variations fluviales du bassin du Mississípi (Annales de Géographie); Sur le mécanisme des transports solides effectués par les rivières ct sur les altérations corrélatives des lits fluviaux (Revne de Géographie Alpine, tome XXIX, 1951).

Covtagne. - Initiation mathématique à l'hydrologie fluviale (conférences à l'Ecole des Ingénieurs Hydrauliciens de Grenoble, 1950).

A. Schlag. - Etude Iydraulique des piscines sur modèles réduits. Exposé des premiers résultats.

A. Schlag et Thmessard. - Premières conclusions de l'étude hydraulique des piscines sur modèles réduits (courbes de concentration).

A. Schlag. - Essais sur des appareils déprimogènes proposés pour la mesure des débits aux petits nombres te Reynolds.

A. Rousserete et A. Schlag. - Contribution à la normalisation des tubes de Venturi classiques.

Quatre ouvrages édités par The New Zealand Institution of Engineers d Wellington :

A. P. Grant. - Channel improvement in fluvial streams (dragage de chenaux dans les flenves a lits affouillables).

A. R. Acheson. - Operation of Totver excavator Otaki River (travaux à la tour excavatrice dans la rivière Otaki).

E. C. Schnackemberi. - Extreme flood discharges (statistique de débits de crue dans diverses parties du monde, et formule de la courbe enveloppe valable pour la Nouvelle-Zélande).

C. J. SeEyle, - The fretuency of heavy rainfalls in New)-Zealand (étude de la fréquence des averses dans la Nouvelle-Zélande, par la formule de la plus grande valeur de Gumbel).

Electricité de France. - Album Equipement, 1950. 


\section{Activité de l'Association}

M. Ie Président rend compte de l'activité du Comité : La Commission de Révision du Code d'Essais des Installations Hydrauliques a continué ses travaux au cours des réunions des 16 mars, 30 avril et 17 mai; quelques retouches ont été apportées aux chapitres relatifs aux méthodes de mesure des débits par exploration du champ des vitesses, par la méthode Gibson et par la méthode chimique; les projets relatifs a la mesure des débits par déversoirs, méthode d'Allen, méthode d'Anderson et appareils déprimogènes seront examinés à la prochaine réunion en vue de faire donner, pour les quatre premiers fascicules du Code d'Essais, homologation par le Conseil d'administration.

La Sous-Section de Glaciologie a tenu sa deuxième réuninon annuelle le 29 mai pour entendre des exposés de: M. SALEs sur ia détection des avalanches.

M. ONDE sur la classification des verrous glaciaires.

M. Wmann sur les observations glaciologiques au glacier Noir et au glacier Blane (massif du Pelvoux).

M. Lugrez sur des travaux glaciologiques américains.

Lecture a été donnée de notes envoyées par :

M. DE Quervar, sur les avalanches de l'hiver 1950-1951 en Suisse.

M. VANNI, sur les variations des glaciers italiens en 1950 . M. Granovx, sur le glacier de la Brenva.

Le programme de la «Tournée Glaciologique» 1951 a été mis au point au cours de cette réunion : cette tournée visitera, les 24,25 et 26 juillet, les glaciers de la Pilatte, dans la vallée du Vénéon, et les glaciers Blanc et Noir dans la Vallouise. Deux groupes convenant aux participants d'endurance différente ont été prévus.

Le «Vocabulaire Technique 》 de la Houille Blanche concernant la glaciologie a été proposé à l'examen de la sous-section compétente.

Une réunion tenue le 2 juin sous la présidence de M. Hupner a abordé « l'étude de la détermination des débits maxima de crues à prévoir pour un barrage et pour lesquels il y a lieu de les ealculer $\gg$. M. Moniat a classé en trois divisions les divers mémoiles présentés à New-Delhi : formules empiriques, méthodes statistiques et méthodes analytiques. M. Gibrat a exposé le parti qu'on peut tirer en ce domaine de la statistique et du calcul des probabilités et M. Paros a exprimé le point de vue des géographes. Les conclusions provisoires ainsi qu'un programme de mise en application des méthodes par Electricité de France à un de ses bassins hydro-électriques, ont été présentés au cours de la session par M. Monlat dans sa communication : « Note sur l'évaluation du débit de crue. »

De nouvelles réunions auront lieu pour discuter certains points particuliers et essayer de formuler des conclusions générales.

Prix d'Hydrotechnique et prix Henri-Milon 1951. Voir palmarès de la S.H.F. page 580 du n" B-1951

\section{Publications de la Société}

M. le Président donne connaissance des publications de la Société :

\section{a) Parues récemment:}

L'Hydraulique des stations limnimétriques pour la mesure du débit des cours d'eau, par M. Réméniéras (tiré à part de l'Annuaire Hydrologique de la France, 1949).

Monographie hydrologique du bassin de la Maronne, par MM. RÉménúris et Boyer (tiré à part de la Honille Blanche, 1950). b) Sous presse (1):

Mémoires et Travaux, $\mathrm{n}^{\circ} \mathrm{I}-1951$.

Slations Hydrométriques Frangaises (2e partie, région du Massif Central).

\section{c) En préparation:}

Quatre fascicules du nouveau Code d'Essais des Installations Hydrauliques.

Annuaire Hydrologique de la France d'Outre-Mer, en collaboration avec le Service des Etudes de la France d'Outre-Mer d'Electricité de France.

\section{Bibliographie}

Un effort a été tenté pour reconstituer la bibiothèque en rassemblant les mémoires présentés au Comité Technique de la S.H.F. depuis sa création, divers travaux provenant du Laboratoire de Beauvert, les dons de Mme MILoN et de M. Eydoux, ainsi que les revues et ouvrages récents reçus en échange de nos publications.

Un fichier avec répertoire, en cours de constitution, comprend déjà un millier de fiches : il est présenté au Comité 'Technique pendant la session à l'entrée de la salle de réunion et se trouve tous les jours au Secrétariat à la disposition de tous nos membres, adhérents et amis.

\section{COMMUNICATION DE M. LACOMBE}

\section{LES FLUCTUATIONS PÉRIODIQUES DES LAMES DÉFERLANTES}

M. le Président dome la parole à M. Gougenhem qui, en labsence de M. LACombe, présente la communication dont nous domons ci-dessous un résumé :

\section{Résumé}

I. - Les courants et oscillations dûs au « ressac » à l'intérieur de certains ports ont été remarqués par mauvais temps sur la côte ouest de la péninsule ibérique par M. IRIbarren [1] ${ }^{*}$ en avril 1948 et Mr. C. K. ABECAssis [2] en septembre 1949, et dans le port de Casablanca au poste d'amarrage qu'occupait en 1942-1945 le JeanBart (bassin Delande). M. Iribarren a constaté que la période du phénomène, 3 à 4 minutes, semble être égale à la période propre du bassin, et, d'autre part, à celle du rythme d'arrivée à la darse des « groupes de vagues» par mauvais temps : cette arrivée de groupes provoque des surélévations de niveau qui constitueraient l'oscillation excitatrice. Mais le mécanisme affectif du phénomène restait à découvrir.

II. - Or, il se trouve que W. H. Munk, en décembre 1949 [3] a présenté sous le nom de « surf beats》(a) un phénomène qui parait pouvoir être rattaché aux précédents. Il s'agit d'oscillations plus ou moins régulières du niveau de la mer au voisinage immédiat de la côte, d'une période de l'ordre de plusicurs minutes, et dont les amplitudes sont, pour le cas étudié par l'auteur, dans le rapport de un à deux avec l'amplitude des fluctuations de hauteur des vagues incidentes. D'une analyse de ces phénomènes avec un enregistreur de houle et un «tsunami recorder \$ (b) MUNK a déduit que les oscillations de 2 à 5 minutes de périodes du niveau de la mer semblent être causées par les fluctuations du trans-

(1) Ces publications sont actuellement parues (N.D.L.R.).

(*) Les crochets renvoient à la bibliographie en fin d'article

(a) Surf beats = fuctuations du deferlement.

(b) Tsunami recorder = enregistreur d'ondes à Iongue période. 
port de l'eau dans la zone de déferlement (c). Les fluctuations dans les valeurs des courants cotiers liés à la houle et aux « rip currents » (d) seraient dûs au mème phénomène.

Ainsi le rivage constitue une source non linéaire rayounant de l'énergie sous force d'ondes à lougue période.

III. - Une étude plus pécente, sisnalée à M. Lacombi par M. le Président Barrition, a paru en 1950 sous la signature de 'Tuckn [5]. Cette étude a été exécutée sur les enregistrements de houle it Peranporth, en Cornoualle. A première vue il apparait une proportionnalité remarquable et indépendante de la période entre l'amplitude moyenne des lames et celle des « long waves (e) $\Rightarrow$ constatées au bout de 4 à 5 minutes après le passage des lames. Cette première constatation a été suivie d'une étude très serrée au moyen d'un appareil mesureur de corrélation. On constate :

$1^{\circ}$ que le facteur de corrélation est particuliement fort quand il y a un decalage de 4 à 5 minutes dans les 2 graphiques, le graphique « onde longue » étant en retard sur le graphique « vagues $\gg ;$

$2^{\circ}$ qu'un groupe de vagues unique produit une onde constituée par une élévation de niveau suivie d'une dépression considérable, puis d'une nouvelle agmentation (le maximum de corrélation correspond a la dépression);

$3^{\circ}$ que la corrélation parait d'autant meilleure que les vagues provienment d'unc plus grande distance (groupes longs et réguliers).

L'auteur estime que l'on pent songer à attribuer la formation de ces «long waves » à la valuation du transport d'eau sous l'effet des groupes de vagues atteignant la cote, mais jl est probablement plus correct et plus conforme aux observations d'attribuer londe longue a l'augmentation brutale du transport d'eau vers la côte dans la zone de déferlement ou près de cette zone: l'accélération correspondante doit etre compenséc par une acélération vers le large; doù la formation de deux crêtes d'onde dont l'une va vers la côte et l'autre vers le large. Ces crêtes laisseraient entre elle un creux.

Lauteur termine en disant que des travaux thericpues considérables sur le transport d'eau dù aux vagues ont été exécutés; mais il y a encore bien des points obscurs dans ce qui se passe réellement. Quoi qu'il en soit la période de ces ondes longues se trouve presque entièrement dans la gamme de 1 à 5 minutes.

Tuckin met en garde le lecteur contre une confusion entre le transport d'eau sous l'effet des groupes au large et le transport d'eau accompagnant le déferlement. C'est précisément le point sur lequel l'intégration de MuNk prête à critique (voir renvoi e page 9). Il ne semble pourtant pas que Tycken ait en en main l'article de Munir sur l'existence d'ondes longues, faite au Congrès d'Oslo en 1948 et la référence rappeléc ici [4].

\section{IV. - Commentame de M. Lacombe}

Si tous les articles cités sont d'accord sur l'origine des fluctuations de niveau à longue période des eaux còtières, le mécanisme même de leur formation n'en ressort pas clairement.

a) Et d'abord il parait y avoir contradiction entre les résultats de Munk et de Tucker puisque, pour le premier, une crête de londe longue correspond an centre des groupes alors que c'est l'inverse pour le denxième auteur. La divergence est considérable. Fst-elle liée à la façon différente dont ces auteurs ont « intégré 》 l'effet

(c) Sous réserve que l'excés d'eat aleule par la méthodo preconise fondésur l'amplitude lue à l'enregistreur do houle) donne efloctivement es transport d'eau.

(d) rip currents = coumants de retour.

(e) long waves $=$ ondes longues. des lames et l'ont comparé aux enregistrements des ondes longues. Les retards de phase imprimés par les appareils enreristreurs de pression aux ondes des deux espèces sont-ils en cause? De nouvelles études sont nécessaires.

Peut-on tenter d'analyser très simplement ce qui se passe?

Quand les trandes lames d'un groupe déferlent, il y a manifestement un déversement d'eau réparti sur 2 ou 3 périodes de houle, temps relativement fable vis-à-vis de la période de l'onde longue. Il doit se former une onde solitaire qui se propage a la fois vers le large et vers la cote. Done le "tsunami recorder 》 doit enregistrer une élévation, un creux, puis une nouvelle élévation. Mais on comprend mal que ce creux puisse, comme l'indique le corrélogramme britannique, être une dépression marquée, par rapport au niveau moyen. Il conviendrait de calculer les volumes d'eau transportés par les deux ondes Iongues et comparer ce volume à celui de la dépression; quant à la possibilité de réflexion sur la côte, il n'y a pas à s'en ćtonner pour une onde de 2 minutes de période et de 0,20 nn de hanteur. Le critère de réflexion de M. IrubarREN $[6]$ donne :

$$
\text { Pente limite }=\frac{8}{120} \sqrt{\frac{0,20}{g}}=\frac{1}{15} \sqrt{0,02}
$$

soit environ $0^{\circ}, 5$. Les plages normales, surtout dans leur partie haute ont des pentes généralement bien supérieures; il faut donc s'attendre à une réflexion.

Le mécanisme proposé, notons-le, n'élimine pas la contradiction entre MuNk et Tucker.

b) D'autre part, en ce qui coneerne la loi de proportionnalité constatée entre l'amplitude des groupes et celle de l'onde longue, on peut tenter de prévoir quantitativement selon les diverses hypothèses faites relativement à la hauteur des brisants, à quelle loi on aboutira en considerant les transports d'eau comme proportionnels au carré de la hauteur des brisants et non au carré de la hauteur des lames au moment de leur passage sur l'enregistreur de houle.

D'après Muxk [7] la hauteur des brisants $\mathrm{H}_{b}$ fournie par des lames d'amplitude $\mathrm{H}_{0}$ an large et de longueur d'onde $\mathrm{L}_{0}$ au rarge est telle que :

$$
\frac{\mathrm{H}_{t}}{\mathrm{H}_{0}}=\frac{1}{5,3 \sqrt[3]{\mathrm{H}_{0} / \mathrm{L}_{0}}}
$$

qui montre que les carrés des hauteurs des brisants sont cntre eux comme la puissance $4 / 3$ des hauteurs des lames initiales.

Le critère proposé par M. Mrcne [8] amène à des graphiques [9] tels que les carrés des hauteurs des brisants sont entre eux comme les puissances 1,5 à 1,6 des hauteurs des lames initiales, au moins pour des lames dont le $\mathrm{L}_{0}$ vaut $200 \mathrm{~m}$ et le $\mathrm{H}_{0}$ varie de $4 \mathrm{~m}$ à $0,50 \mathrm{~m}$.

Donc, considérant les transports d'eau comme proportionnels au carré de la hauteur des brisants, loi à laquelle conduit l'assimilation à l'onde solitaire limite (voir MUNk [7]) on doit aboutir, si le mécanisme du transport d'eaù est à l'origine des ondes longues, à une loi telle que lamplilade de londe longue varie comme la puissance 1,33 on 1,5 de l'amplitude des lames incidentes. C'est lo moment de se rappeler la difficulté de définir celle-ci avec précision et c'est là peut-être qu'il faut trouver la raison de l'écart entre la loi expérimentale et la loi théorique dans l'évaluation de « l'amplitude moyenne $\gg$ de la houle incidente ou entre, comme dit MUNk, une grande part d'appreciation personnelle. On peut en conclure que l'hypothèse de la formation des ondes longues par le transport d'eau au moment du déferlement est plausible, sinon juste. Elle met en tout cas en cause des phénomènes essentiellement non linéaires qu'il est fort malaisé d'analyser avec précision, non seulement en raison de la difficulté théorique qu'en com- 
porte l'étude mathématique mais aussi de la difficulté d'interpréter les résultats donnés par les instruments de mesures spéciaux qui d'ailleurs ne sont guère employés en France.

Pour terminer on ne peut que rendre hommage a l'esprit d'observation et à l'intuition de M. TRBBAREN, qui a été, semble-t-il, le premier à signaler ces phénomènes et a les rattacher à la séquence des groupes de vagues.

\section{Bibliographie}

[1] R. Imbarten Cavanilies. - Courants et oscillations dus au ressac à l'intérieur des ports. Revista de Obras Publicas, Madrid, avril 1948.

[2] C. M. Abecassis. - Le port de Leixoes. Rapport présenté au XVII Congrès International de Navigation, Lisbonne, septembre 1949, section II, communication IV, 155, rue de la Loi, Bruxelles.

[3] W. H. Munk. - Surf beats. Transactions American Geophysical Union, vol. 30, $\mathrm{n}^{\circ}$ 6, décembre 1949 , pp. $849-854$.

(4) W.-H. MUnk, H.-V. Iglesias et T.-R. Folsom. -- An instrument for recording ultra long frequency waves. Rev. Sci. Ins.. vol. 19 , pp. 654-658, octobre 1948.

[5] M. J. Tucker. - Surf beats; Sea waves of 1 to 5 minutes period. Proc. Roy. Soc., Londres, 22 août 1950.

[6] IR. Iribarnen Cavanilles et C. Nogates y Olaxo. Talud limite entre la rotura y la reflexión de las olas. Revista de Obras Publica, Madrid, février 1950.

[7] W. H. Munr. - The solitary wave theory and its application to surf problems. Annals of the New York Academy of Sciences, mai 1949, pp. 376-424.

[8] Miche. - Mouvements ondulatoires de la mer en profondeur constante et décroissante. Annales des Ponts et Chaussées, janvier-août 1944.

[9] Pour le détail des graphiques voir Lacome : la Houle $\dot{a}$ la côte. Conférence $\mathrm{n}^{\circ} 11 \mathrm{du}$ C.R.E.O. 24 mars 1950 .

\section{Discussion}

M. le Président remercie M. Lacombe d'avoir analysé et critiqué la pensée des trois auteurs cités et M. Got:cEnhem d'avoir présenté cette analyse et la critique de M. LACOMBE.

Sur la demande de M. le Président, M. Grober précise que l'appareil destiné à Tamatave par le Laboratoire National d'Fydraulique est en cours de mise au point; on commencera à faire des mesures dans six mois environ.

M. Ch. BEAU rattache à ces observations les phénomènes de marées en profondeur constatés par la Société " l'Energie des Mers » dans la vallée sous-marine de la Cote d'Ivoire appelée "Trou sans fond 》; ces mouvements affectent des masses d'eau plus considérables que ce que l'on prévoyait et ont des conséquences inattendues : il y a d'abord des phénomènes à longue période qui correspondent à peu près aux marées avec certains décalages et qui créent des courants de l'ordre de $20 \mathrm{~cm} / \mathrm{s}$ à $400 \mathrm{~m}$ de profondeur; d'autre part, on constate que les écarts de température au cours de la journée varient, par exemple à $350 \mathrm{~m}$ de profondeur, de $7^{\circ}, 5$ à $10^{\circ}, 5$; ce qui correspond à des espèces de vagues, en profondeur, un peu analogues à ce que l'on avait trouvé à Grenoble dans les études sur modèle réduit pour se rendre compte du fonctionnement d'une prise d'eau à grande profon- deur. De plus, il semble qu'il y a des ondulations plus faibles qui correspondent peut-ètre, et jusqu'à une certaine profondeur, à des phénomènes de forte barre agissant pendant quelques minutes, non seulement en surface, mais également à une certaine profondeur.

M. Govgenhem dit qu'il serait intéressant de rechercher en premier licu les périodes des phénomènes parce que l'amplitude des ondulations à l'interface de deux milieux est inversement proportionnelle à la différence de leurs densités, et peut donc atteindre une valeur relativement élevée lorsque ces densités sont voisines. Il y a à noter que les différences des vitesses horizontales entre les moments des crêtes et des creux penvent paraitre doubles du fait que ces vitesses sont à ces moments en opposition.

M. DE Rouvilie remarque que si les vagues penvent ètre influencées par le « Trou sans fond 》, la marée ne peut guère l'être par un accident aussi petit par rapport à son échelle; d'autre part, il suggère de sréer une liaison entre le Service Hydrographique et le Comité d'Etudes de l'Energie des Mers en vue d'un programme de mesures plus scientifiques.

iT. Govgenhem croit, toutefois, que ia configuration du «Trou sans fond 》 est telle que les courants ascendants ont certainement une importance.

M. Pierre Cor fait observer que le ressac se constate dans certains ports, même en l'absence de brisants : pa.' exemple à Madras, où la côte de Coromandel est plate et au Havre même dans le bassin de marée. Il semble que le phénomène soit causé dans ce cas par l'introduction - périodique ou non - de masses d'eau propulsées par des phénomènes divers tels que des houles cycloniques de très grande longueur d'onde ou plus exactement par de petits raz-de-marée successifs. Il se produit une mise en mouvement, puis une oscillation de la masse liquide du bassin. Des déferlements sur des brisants peuvent d'ailleurs produire les mêmes effets.

M. Govgenhem rappelle que dans lanalyse de M. LACOMbe il y a deux choses distinctes : l'existence à la côte d'unc oscillation à lơngue période entretenue par le déferlement, et l'agitation périodique dans les ports, liée, selon M. Irmarrex, à l'existence des groupes de lames.

M. WALIEr signale l'intérêt qu'il $y$ a à produire expérimentalement en Laboratoire des trains de vagues pour étudier leur effet sur certains phénomènes particuliers: houles à variation de période continue pour l’étude de la gifle, dont les circonstances de formation sont très étroites; trains de vagues d'amplitude croissante ou décroissante, ou de périodes variables pour l'étude de la stabilité d'un ouvrage, on de l'agitation dans un port. Le Laboratoire Dauphinois d'Hydraulique a ralisé un certain nombre d'apparcils grâce auxquels on produit ces trains de vagues, ainsi qu'un dispositif permettant de faire varier les caractérisiques de la houle on marche et à distance dans un canal vitré, à partir de la vitre d'observation par l'intermédiaire d'un servo-moteur; ce dispositif sera décrit tout à l'heure (1).

M. Garier ajoute qu'il serait intéressant d'avoir une communication sur les essais faits entre 1940 et 1944 à Grenoble sur des ondes entretenues dans des dièdres par des trains de vagues, et où l'on voit que le comportement de la vague dans le dièdre peut présenter deux modes différents : ou bien propagation pure et simple avec augmentation de l'amplitude de la vague dans le fond du dièdre, ou bien entretien d'une seiche.

M. Govarnherm confirme que les trains d'onde de la houle sont très utiles à étudier, notamment pour l’analyse de la propagation de l'énergie de la houle.

Une controverse s'engage au sujet de la définition du

(1) Voir plus loin communication de MM. Breslir of WArrest : Description d'une installation d'études expérimentales sur la physique de la houle. 
mot « ressac » demandée a $M$. Cot par M. Gariel; selon M. Grider, le mot paraît avoir la mème étymologie que le mot seiche, dont il est la traduction en espagnol (resaca); il est employé par les marins dans le sens de seiche ou de clapotis.

M. le Président signale, à ce sujet, l'intérêt du vocitbulaire proposé par M. Garies, et demande que les mots seiche, ressac, clapotis et clapotis gaufré y soient définis, dans la mesure où ces termes correspondent à des réalités tangibles dans les mers ou les lacs.

En ce qui concerne les phénomènes de seiche dans les dièdres M. le Président rappelle que c'est le cas le jlus compliqué des seiches et qu'il sera intéressant d'entendre la communication proposée par M. Ganiel. Il en sera de même pour les appareils générateurs de houle qu'on pourra passer en revue.

Sur une question de M. le Président, M. RÉméñénas prècise que certains essais qu'il a effectués en 1942 au Laboratoire de Beauvert de la S.H.F. se rattachent au même sujet. Ils ont comporté l'étude de l'augrmentation d'amplitude d'une onde de translation au cours de sa progression vers le sommet d'un dièdre; la surélévation du plan d'eau dans diverses sections du dièdre a pu etre calculée avec une bonne approximation, par une méthode graphique (dérivée de celle de M. Bergenon), tant que les « courbures » n'étaient pas trop fortes. En metani en jeu un phénomène de résonance on a pu obtenir au sommet du dièdre des ondes de hauteur plus de dix fois supérieure à celle qu'elles présentaient à l'entréc et réaliser ainsi une sorte de « bélier hydraulique à ciel ouvert $»$.

En ce qui concerne le vœu exprimé par M. DE Rouville, M. Govgenhem estime que la liaison entre le Service $\mathrm{Hy}$ drographique et l'Energie thermique des mers existe déjà.

M. Gariel indique que des essais sont en cours au Laboratoire Dauphinois d'Hydraulique sur des phénomènes de seiche d'acier fondu qui se produisent dans certaines conditions dans les convertisseurs Bessemer.

\section{COMMUNICATION DE M. BIESEL}

PRÉSEnTÉE Par M. WALLE'l

\section{ÉTUDE THÉORIQUE DE LA RÉFLEXION DE LA HOULE SUR CERTAINS OBSTACLES}

\section{Résumé}

Gertains obstacles, par exemple une digue immergée, réfléchissent une partie de la houle, et en transmettent un autre fraction. La présente étude théorique valable au premier ordre, montre qu'on peut disposer plusieur's de ces obstacles les uns derrière les autres, de façon que pour une houle de longueur domnée, il n'y ait plus aucune réflexion. Un exemple d'une telle disposition est le suivant :

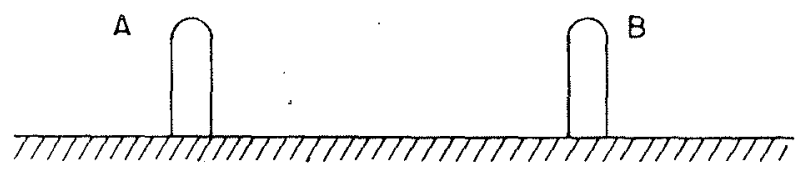

FIG. 1.

les deux obstacles étant identiques et symétriques, et séparés par une distance déterminée.

Ainsi, on peut éliminer les réflexions causées par un obstacle tel que A en plaçant soit devant, soit derrière un ou plusieurs obstacles réfléchissant convenablement calculés.

Pour étudier commodément l'influence d'un ou plusieurs obstacles sur la houle, celle-ci est rapportée it un plan de référence lié aux obstacles : une houle est alors représentée par l'amplitude verticale qu'elle crée dans ce plan, aussi bien en ce qui concerne l'intensité de cetto amplitude que sa phase. Une houle est donc caractérisée par un vecteur tournant (de mème qu'un courant alternatif en électricité). Cet artifice permet de comparer les phases des houles incidentes et réfléchies. Il prèsente l'avantage de conduire d'une manière très simplo aux r'ésultats, ceci sans écrire les équations intrinsèques du phénomène.

Si un obstacle possède un coefficient de transmission $\alpha$ et un coefficient de rétlexion $\beta$, nous pourrons, en supposant que la transmission et la rétlexion s'opèrent avec des déphasages $\alpha$ et $\widehat{\beta}$ représenter l'influence de l'obstacle sur la houle par les deux nombres complexes $\bar{\alpha}$ et $\bar{\beta}$. Si le nombre complexe A représente l'emplitude et la phase de la houle incidente, les houles transmises et réfléchies seront respectivement représentées par les nombres complexes $\vec{\alpha}$ A et $\bar{\beta} A$.

Ceci permet de mettre en évidence une propriété importante des obstacles. Par de simples considérations énergétiques, s'exprimant par des relations telles que :

$$
\alpha^{2}+\beta^{2}=1
$$

on parvient, après plusieurs artifices de calcul, au x'ésultat suivant: tout obstacle, même dissymétrique, a des coefficients de transmission el de réflexion qui, en valeur absolue, ne dépendent pas du sens de la houle incidente.

En considérant maintenant la combinaison de deux obstacles symétriques, la méthode de calcul permet, connaissant les nombres $\bar{\alpha}$ et $\bar{\beta}$ relatifs à chacun d'eux, de déterminer toutes les houles intervenant dans le phénomène. Elle permet notamment d'écrire la condition nécessaire pour que l'ensemble ne soit pas réfléchissant, ce qui impose l'égalité en valeur absolue des pouvoirs réfléchissants des deux obstacles et définit la distance $\frac{\theta L}{2 \pi}$ qui doit les séparer, $L$ étant la longueur d'onde de la houle. Le calcul donne en effet pour $\theta$ la valeur :

$$
\theta=\frac{\widehat{\beta_{1}}+\widehat{\beta_{2}}}{2} \text { à } \pi \text { près. }
$$

$\widehat{\beta_{1}}$ et $\widehat{\beta_{2}}$ sont les arguments de $\overline{\beta_{1}}$ et $\overline{\beta_{2}}$ c'est-áa-dire les déphasages apportés par les réflexions sur chacun des deux obstacles.

Ces considérations théoriques, qui démontrent la possibilité d'annuler les réflexions d'un obstacle par l'adjonction d'un deuxième obstacle réflèchissant, sont susceptibles d'applications pratiques.

On peut, par exemple, améliorer l'amortisseur d'extrémité d'un canal à houle, en lui associant un obstacle ayant même pouvoir réfléchissant que lui en valeur absolue.

Par ailleurs, un filtre ne supprime pas complètement les réflexions provenant des ouvrages à étudier : elles se réfléchissent sur le batteur et, après un deuxième passage à travers le filtre, retournent vers le modèle. L'ensemble batteur-filtre se comporte donc comme un amortisseur imparfait, qu'on peut donc également améliorer par l'adjonction d'un obstacle réfléchissant.

\section{Discussion}

M. le Président remercie M. Wallet.

Sur une demande de M. BEAu relative à la possibilité 
de réaliser pour des ports ou des hydrobases des dispositifs permettant de tranquilliser un plan d'eau calme au moyen de digues ou de tranchées sous-marines, M. Wallet précise que to calcul de M. Buskl n'est valable que pour une longueur d'onde donnéc; que d'autre part, les phénomènes secondaires de tension superficielle et surtour de viscosité, négligés dans la loi linéaire précitée sont quelquefois importants dans la réalité; ils domnent lieu à une dissipation d'énergie dont les effets peuvent d'ailleurs dans certains cas s'ajouter a cenx prévus dans la thérie de M. Bieser.

M. Gariel ajoute à ce sujet que ces phénomènes n'ont été étudiès jusqu'à présent qu'en vue d'applications au laboratoire, que l'étude des mèmes phénomènes dans la nature en découlera mais n'a pas encore élé entreprise.

M. Bead ayant demandé s'il existe un rapport entre le procédé exposé par M. BuEsel et celui dit « de la réllexion totale $\gg$ dont une réalisation sous la forme de fossés sous-marins obliques à la houle a été préconiséc par divers auteurs et entre autres à la S.H.F. par H. CanLotru comme le rappelle M. Gougexhesm, M. Griber, indique que ces deux dispositifs lui semblent de nature tout à fait différente.

Le procédé de la réflexion totale consiste à combiner l'incidence de la houle sur une cote avec un changement brusque de la célérité (provqquée par une variation brusque de profondeur) pour obtenir un reflux de l'énergie en dehors de la zone côtière à protéger; il n'y a donc pas dans ce cas destruction d'energie, mais simplement renvoi de celle-ci vers le large.

Le procédé présenté par M. Busser consiste à provoquer par des réflexions frontales partielles deux clapotis d'importance égale déphasés de telle facon que leurs effets s'annulent: il y a donc dans ce cas destruction de l'énergie.

M. Waller fait remarquer à ce sujet que lout accident du fond s'accompagne d'une réflexion de la houle. Les obstacles réfléchissants de la thérie de M. Breser. peuvent donc ctre positifs ou négatifs, e'est-it-dire constitués par une paroi ou une tranchée notamment.

M. Devineux précise que les recherches poursuivies par M. Canlotrl et auxquelles M. Governhem a fait allusion précédemment ont été réalisces au Laboratoire Central d'Hydraulique a Maisons-Alfort el que la publication des résultats en a été faite il $y$ a phusicurs années dans la Revue génćrale de lingdraulique.

M. Devinevx ajoute que le probleme de la eríation de ports artifieiels dépasse largement la question de la défense contre la houle et que ce problème fait actuellement l'objet d'études et d'essais en cour's d'execution au Laboratoire central d'Hydraulique potr le comple de l'Administration.

M. Warret est bien d'accord avec M. Devmeux of signale que le Laboratoire Dauphinois d'Hydraulique itudie des dispositions faisant intervenir des principes différents de ceux qui font l'objet de la communication de M. Biessi et de la sienne.

COMMUNICATION DE M. WALLET

\section{DESCRIPTION D'UN DISPOSITIF EXPÉRIMENTAL POUR L'ÉTUDE PHYSIQUE DE LA HOULE}

\section{Résumé}

Les études faisant intervenir la houle expérimentale produite en canal concernent le plus souvent des maquettes d'ouvrages en projet, dont il s'agit de definir les qualités : stabilité, absence de franchissements, pouvoir absorbant, parmi les principales.
Dans ce cas. la production d'une houle de caractéristiques constantes dans le temps, permet si l'on évite des réllexions trop importantes sur le système génélateur de houle, d'obtenir des résultats en général suffisamment fidèles pour qu'on puisse comparer entre eux differents types d'ouvrages.

Cependant ces conditions sont nettement insuffisantes lorsqu'on étudie la physique de la houle, éest-i-dire lorsqu'on veut mettre en évidence, avec une précision sulfisante, la structure même et les caractéristiques du phénomène de la houle, afin de pouvoir notamment comparer ces résultats avec les domnées de la théorie.

Il faut alors un outil expérimental beaucoup mieux aldapté à ce genre d'études. C'est une longue suite de perfectionnements, dont nous allons décrire ici les plus caracteristiques, qui a permis d'obtenir sur l'une des installations du Laboratoire Datuphinois d'Hydraulique, une houle particulièrement pure et constante.

Cette installation devait offuir le maximum de possibilités expérimentales, notamment pour l'obtention des houles fortement cambrées. On admet on général que ceci est incompatible avec l'emploi de filtres néccssaires pour absorber les réflexions sur le batteur, mais qui absorbent également une partie de l'énergie de la houle incidente.

Le problème a été résolu par l'emploi d'un convergent placé entre le batteur à houle et le canal d'études. Ce convergent « concentre » en quelque sorte l'energie de la houle et renfore son amplitude.

Lat convergence a été prévue de manière à compenser la perte d'énergie dans les filtres, ainsi que l'amortissement propre.

Le convergent qui a été réalisé est extrêmement progressif, puisque sa longueur est de $11 \mathrm{~m}$, pour une varialion de largeur de $0,45 \mathrm{~m}$. Cette condition était nécessaire, car une variation brutale de section entraîne des perturbations importantes dans la propagation de la houle : lorts balancements transversatux, reflexions, elc... L'installation comprend alors :

Le batteur à houle, les filtres, le convergent, le canal d'etudes proprement dit où se trouve la partie vitrée, at l'amortisseur d'extrémité. Cet ensemble est complèté par une conduite d'équilibrage dont nous expliquerons l'utilité.

Le batteur a houle est d'un type particulier, mis au point par le Laboratoire Dauphinois d'Hydraulique. Il est en effet conçu de telle façon que son mouvement puisse varier de façon continue depuis l'oscillation simple autour d'un axe horizontal, jusqu'a la translation, en passant par tous les intermédiaires. Le mouvement du batteur peut ainsi s'adapter à celui des differentes houles qu'on veut produire en canal. La translation en particulice est nécessaire pour les houles de glande longueur d'onde en eau peu profonde.

Un autre trait original de ce batteur est que les différents paramètres peuvent être réglés à distances te qui permet de modifier en marche, à partir de la vitre d'observation, la période et l'amplitude de la houle.

Les filtres sont du type ì plaques perforées. Cependant un autre genre de filtre a éte mis au point, qui donne dès maintenant des résultats particulièrement intéressants. Il est constitué par du grillage plié et soudé de manière à constituer un ensemble d'alvéoles. Le filtre est alors très rigide tout en étant léger et diffus : il n'apporte pas la moindre perturbation à la houle incidente.

l'absorption d'énergie, se fait en effet sur une grande longueur et l'on n'a pratiquement plus de réflexion résiduelle comme il s'en produit parfois sur les filtres trop compacts qui présentent un plan privilésté.

Le convergent dont nous avońs parlé plus haut est utilement complété par une série de guideaux longitudinaux évitant les légers balaneements transversaux qui pourraient se produire pour certaines périodes particulières. 
Un filtre finissen de pouroir amortisseur faible peut être alors disposé entre le convergent et la vitre d'observation, ceci afin d'améliorer encore le profil de la houle. La partie rectiligne faisant suite au convergent comprend un élement vitré permettant les observations et les photographies.

Le canal est également muni d'une conduite d'équilibrage nécessaire lorsque, dans l'étude d'un ourrage. celui-ci est franchi par la houle qui tend ainsi it creer peu à peu derrière l'ouvrage une mise en charge nuisible. La conduite permet alors de faire communiquer la région situé derrière louvage avec celle se trourant au voisinage du batteur. Des précautions spéciales doivent etre prises pour que le débit apporté par la conduite d'équilibrage ne perturbe pas la houle.

Le canal rectiligne comporte à son extrémité un amortisseur. Celui-ci est constitué par une plage perméable de grande longueur en gravier, précédée de plusieurs filtres. La perméabilité du gravier permet dobtenir une très bonne absorption d'énergie par un froftement intense à l'intéricur mème de l'amortisseur. L'effet de cette plage, joint à celui des filtres, permet ainsi d'éliminel presque complétement les réflexions à l'extrémité du canal. Ceci est suffisant pour un grand nombre d'études: mesure de célérités, mesures de courants de transports, etc... Cependant la légère réflexion résiduelle est gênante pour certaines etudes très poussées sur la structure même de la houle, comme des recherches sur la forme des profils ou des trajectoires internes. On a alors mis au point un procédé permettant d'annuler cette dernière réflexion, en accord avec la théorie exposée dans la communication de M. Bisser. (Etude théorique de la réflexion de la houle sur certains obstacles). A la plage, considerée comme un réflecteur partiel, est associé un obstacle de mème pouroir réfléchissant, en l'occurence une plaque verticale partiellement immergée.

La position de cette plaque est telle qu'a un noud (ou plutôt psendo-nœud) du clapotis partiel créé par elle, correspond un ventre du clapotis partiel créé par la plage. Ouant à l'enfoncement de cet obstacle. il est réglé expérimentalement de manière que l'enveloppe supérieure de la ligne d'eau se rapproche le plus possible d'une droite. On parvient de cette manière à un pouvoir réfléchissant inférieur ì $1 \%$.

Cette installation a domne lieu it plusieurs itudes experimentales, dont certaines sont actuellement en cours. Citons notamment : l'étude des profils de houle, celle des trajectoires internes de la houle et du clapotis et la comparaison de ces résultats avec les données théoriques, l'étude des trajectoires internes dans un clapotis partiel (photographies publiées dans la Houille Blanche), étude de la célérité de la houle, étude du clapotis limite, etc..

\section{Commentaire}

M. le Président remercie M. Walcer et souligne la valeur des clichés de propagation de houle projetés au cours de la communication : ces clichés sont trés descriptifs et font honneur a la science française.

La séance est levée à 12 heures.

\section{SEAANCE DU MARDI APRES-MIDI 19 JUIN 1951}

La séauce est ouverte à $14 \mathrm{~h}: 30$, sous la présidence de N. Garnes, Président de la Société Hydrotechnique de Frauce.

La parole est donnée à M. Silber.

\section{COMMUNICATION DE M. SILBER}

\section{DIAGRAMME SYNTHÉTIQUE \\ DES ÉCOULEMENTS GRADUELLEMENT VARIÉS EN CANAUX DÉCOUVERTS ET DIFFÉRENTES FORMES DE SURFACE LIBRE.}

\section{Résumé}

Tous les auteurs classiques indiquent que les courbes de la profondeur d'ean $y$ en fonction de l'abscisse $x$ me tendent vers une tangente horizontale que lorsque la profondeur d'eau tend vers l'infini. Dans une étude récente (1), M. Srberr a reproduit egalement cette forme usuelle.

Cependant Mocher (2) a montré qu'il y a dans le cas d'un canal rectangulaire infiniment large à coefficient de CHÉzx non constant, une profondeur "caractéristique »non infinie pour laquelle la tangente à la ligne d'eau est horizontale. Plus récemment, M. A. LAzARD (3), ingénieur en chef des Ponts et Chaussées, reprenant l'étude de Mou-

(1) R. Stzber, - Etude des ecotulements graduellement variés en canaux découverts. Mémoires et Trabaux de la S.H.F. année 1950 , vol. II.

(2) Mouret. - Cours de l'Ecole des Ponts et Chausses. 1947
RET, a montré, en traitant le cas du canal rectangulaire non infiniment large et celui du canal circulaire, qu'il pouvait exister deux profondeurs caractéristiques non infinies, à condition que la pente du canal soit supérieure à une pente appelée par lui «pente limite».

M. Silmer a cherché dans la présente étude à déterminer les raisons et l'importance de ces anomalies et ia classer systématiquement les lignes d'eau en dégageant les critères gouvernant leurs variations. Il à été ainsi

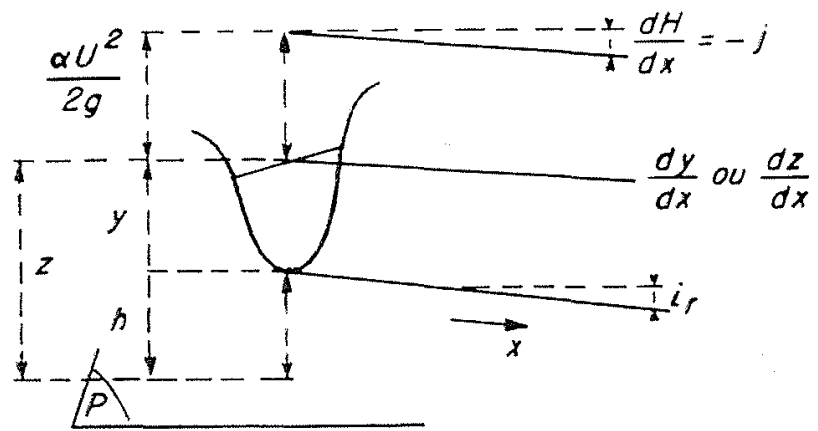

FIG. 1.

amené à établir un diagramme synthétique qui se prête particulièrement bien à l'étude de ces différentes formes, à leul classification et à la mise en évidence de ces critères ainsi qu'à celle de l'influence de la forme des sections du canal prismatique.

Les équations de ce diagramme sont obtenues en dérivant l'équation fondamentale classique des écoulements graduellement variés (fig. 1) et en dégageant trois paramètres : la pente du radier $i_{\gamma}$, la pente de la ligne de 
charge $j$ et le nombre de Froube F. Si lon appelle $y$ la protondeur d'au et z la cote de la surface libre audessus du plan de référence, on oblient:

$$
\begin{aligned}
& \frac{d y}{d x}=i_{r} \frac{1-j i_{r}}{1-F} \\
& \frac{d z}{d x}=i_{r} \frac{1-j-i_{r}}{1-F^{2}}
\end{aligned}
$$

Lammulation des termes de ces fractions correspond à diverses conditions: profondeur nomale (ecoulement uniforme), profonden critique $y_{c}$, profondeur caractéristique de Nounet $\left(y^{\prime}\right.$ ou $\left.y^{\prime \prime}\right)$. Le diagramme synthetique est construit en prenant $\mathrm{F} z$ pour abscisse et $j / i$, pour ordonnees. Les droites d'abscisse et d'ordonnées égales a l'unite divisent le diagramme en quatre zones correspondant à diverses conditions d'éconlement (fics. 2) et la caractéristique d'un canal donné pour un débit domné et une pente donnec a l'allure d'une des courbes de la figure 3 ; en effet :

$$
\begin{gathered}
\frac{j}{i_{r}}=\frac{\mathrm{Q}^{2}}{\mathrm{C}^{2} \mathrm{~S}^{2} \mathrm{R}_{\mathrm{F}} i_{r}}=\frac{\mathrm{C}^{2}}{\mathrm{C}^{2} \mathrm{R}_{\mathrm{F}^{i}}} \\
\mathrm{~F}^{2}=\frac{\mathrm{C}^{2}}{g y_{m}}=\frac{\mathrm{Q}^{2}}{g_{1} !_{m} \mathrm{~S} 2}
\end{gathered}
$$

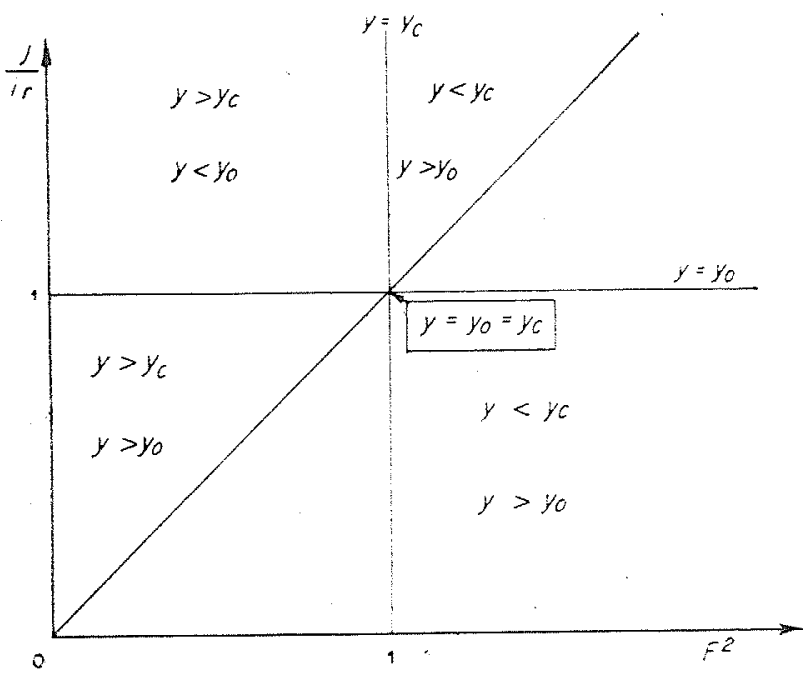

Fig, 2.

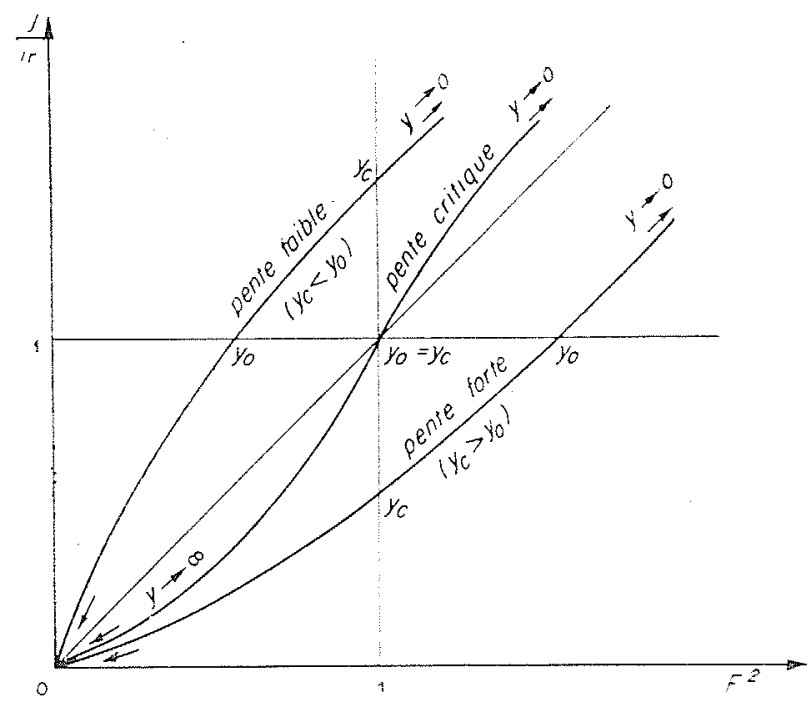

Fig. 3.
Le lieu des points a tangente horizontale est la droite de pente egale a lunité. La caracteristique pourra couper cette droite on un point ou plusicurs points $j / i_{r}=\mathrm{F}_{2}$ defoissant les protondeurs $y^{\prime} y^{\prime \prime}$.. . correspondant a une tangente horizontale (fig. 4). On ealcule aisement que ces profondeurs sont celles pour lesquelles la pente critique $i$ e est esalo a la pente du radier :

$$
i_{c y}=i_{c y,}=i_{r}
$$

la pente critique etant définie par:

$$
\dot{i}_{c y}=\frac{g y_{m}}{\mathrm{C}_{2} \mathrm{R}_{\mathrm{HI}}}
$$

Les equations parametriques des cameteristiques peuvent secrire, en se réferant aux valeurs correspondant a la profondeur nomale $\left(i_{r}=j\right)$ et en introduisant la débilance $\mathrm{k}$ de BAKHMETEF :

$$
\begin{aligned}
& \frac{j}{i_{0}}=\left(\frac{K_{0}}{K_{i}}\right)^{2} \\
& F_{2}=\frac{i_{j}}{i_{e !}}\left(\frac{K_{0}}{K}\right)^{2}
\end{aligned}
$$

En appliquant ces equations a divers cas de canaux prismaticues, M. Srben trouve :

A. - Pour les canalux de section reclangulaire infiniment large à coefficient de CHézr constant (cas de Bresse), une caractéristique représentée pour la pente critique par la droite de pente unité, independante de la rugosité et du débit (résultat classique rai uniquement pour le cas de Bresse); pour d'autres pentes $i_{r}$ données, le réseau des caracleristiques est constitué par un faisceau do droites issues de l'origine et l'ordonnee du diagramme peut ètre graduée en $y / y_{0}$; dans tous les cas le seul point de tangente horizontale est à l'infini.

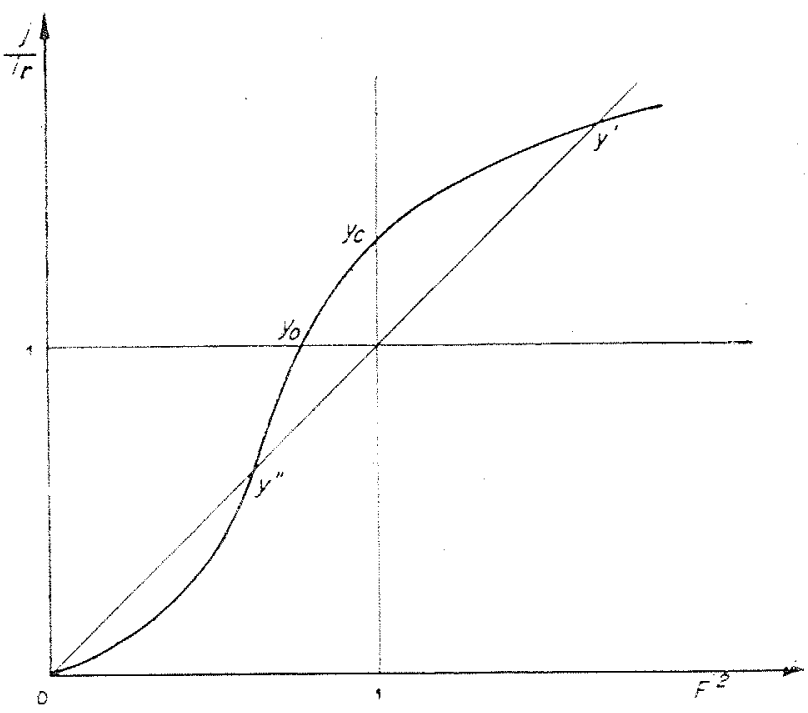

Fig. 4

B. - Pour les memes ranaux que ci-dessus mais i coefficient de CHËzy non constant (cas de Mounet), les catracteristiques sont des courbes homothétiques; celle qui correspond à la pente critique est tangente à l'origine à l'axe des abscisses et ne rencontre la droite de pente unité qu'au seul point d'abscisse et d'ordonmée unité Les autres coupent cetle droite en un seul point correspondant à un point à tangente horizontale de la ligne d'eau, et appartenant au régime fluvial pour une pente faible et au régime torrentiel pour une pente forte.

C. - Ponr un canal non infiniment large, les caractéristiques sont egalement des coupbes homothetiques: 
celles qui correspondent à la pente critique sont tangentes à l'origine à l'axe des ordonnées, passent par le point d'abscisse et d'ordonnée unité, et présentent un point d'inflexion au-delà ducquel leur concavité est tournée vers le haut, comme celle relative à la largeur infinie: chacune d'elles coupe donc la droite de pente unité en un seul point, outre celui d'abscisse et d'ordonnée unité; ces points de tangente horizontale sont situés en régime nuvial pour les canaux de grande largeur et en régime torrentiel pour les canaux de faible largeur; pour la valeur critique $y_{0} / l=\frac{1}{6}$ (largeur critique (cette valeur $1 / 6$ étant due au choix de la formule de Manniva pour la variation du coefficient de ChĹzy), la courbe est tangente à la droite de pente unité au point d'abscisse et d'ordonnée unité. Pour des pentes non critiques, le réseau des caractéristiques comprend une double infinité de courbes dépendant des paramètres $y_{0} / l$ et $\frac{i_{r}}{i_{o y}}$; cette double infinite est mmenée à une seule infinité en remplaçant le paramètre Fo par $\frac{i_{c y_{0}}}{i_{r}} \quad \mathrm{~F} 2$.

La variation de la fonction $-\frac{i_{c y_{1}}}{i_{c y_{0}}}$ permet d'étudier la forme des caractéristiques. Chaque courbe passe par un minimum pour la valeur $\frac{y}{y_{0}}=\frac{1}{6 y_{0}}$, soit pour $y / l=\frac{1}{6}$. Il existe une valeur minimum de la pente $i_{r}$ correspondant à une valeur minimum de $\frac{i_{r}}{i_{c y_{0}}}$ au-dessous de laquelle la droite lieu des points à tangente horizontale de la ligne d'eau ne coupe pas la caractéristique. M. Lazard l'avait indiquée et appelée pente limite.

Lorsque la pente du radier est supérieure à cette pente, la droite coupe la caractéristique en deux points, cor- respondant à deux points à tangente horizontale de la ligne d'eau. La figure 5 indique ces différents domaines dans les trois cas du canal de grande largeur, de largeur eritique et de faible largeur.

Les diffèrentes courbes de surface libre peuvent être déduites de cette classification des profondeurs $y_{0}, y_{c}$, $y^{\prime}$ et $y^{\prime \prime}$. La pente de la surface libre par rapport au radier $\frac{d y}{d x}$ est proportionnelle à $\operatorname{tg} \alpha$ ou $\operatorname{tg} \alpha^{\prime}$, si l'on désigne par $\alpha$ dans le diagramme $\left[j / i_{\gamma}, F\right]$ ou $\alpha^{\prime}$ dans le $\left[j / i_{r}, i_{c y_{0}} F_{2}\right]$ l'angle que fait le rayon vecteur issu du point d'abscisse et d'ordonnée unité et aboutissant au point courant $M$ de la caractéristique :

$$
\begin{aligned}
& \operatorname{tg} \alpha=\frac{1-j / l_{r}}{1-\mathrm{F}^{2}} \\
& \frac{d y}{d x}=i_{,} \operatorname{tg} \alpha \\
& \operatorname{tg} \alpha^{\prime}=\frac{i_{r}}{i_{c y_{0}}} \frac{1-j / i_{r}}{1-\mathrm{F}^{2}} \\
& \frac{d y}{d x}=i_{c y_{0}} \operatorname{tg} \alpha^{\prime}
\end{aligned}
$$

le coefficient de proportionnalité étant soit la pente du radier, soit la pente critique pour la profondeur normale.

L'auteur analyse ensuite complètement le cas particulier d'un canal de grande largeur et de pente faible en faisant décrire au point courant $M$ la caractéristique depuis le point à l'infini $(y=0)$ jusqu'à l'origine $(y=\infty)$. On retrouve dans cette analyse le « creux $»$ et le «sommet », points à tangente horizontale pour le profil. Pour le canal circulaire, le minimum de la fonction $\frac{i_{c y}}{i_{c y_{0}}}$ est donné pour $y / r=0,59, r$ étant le rayon $d u$
Conal de grande lorgeur

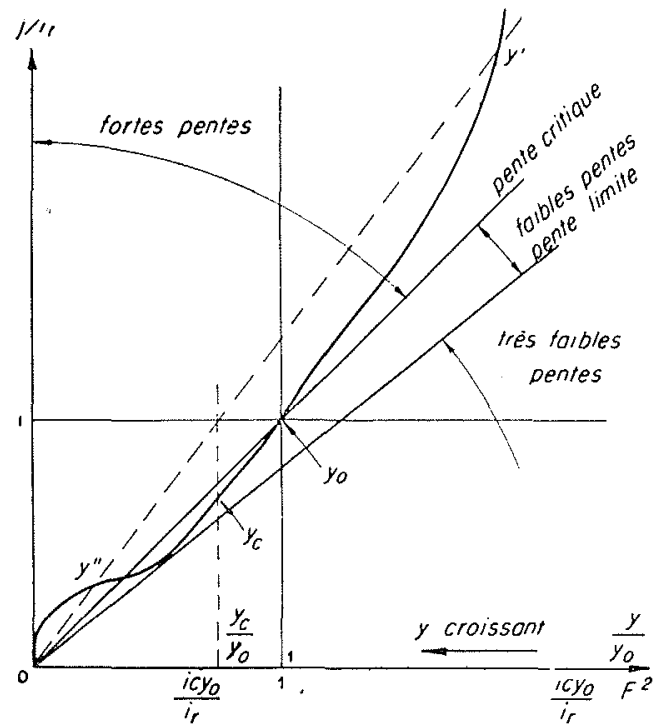

Pente forte......... $y^{\prime}<y_{0}<y_{c}<y^{\prime \prime}$

Pente critique....... $y_{c}=y_{0}=y^{\prime}<y^{\prime \prime}$

Pente faible......... $y_{c}<y_{0}<y^{\prime}<y^{\prime \prime}$

Pente limile......... $i_{\gamma}=i_{c y}$ minimum

Pente très faible.... $\begin{aligned} & y_{c}<y_{0} \\ & y_{c}<y_{0}\end{aligned}$ $y_{c}<y_{0}<y^{\prime}=y^{\prime \prime}$

$\left(y^{\prime} y^{\prime \prime}\right.$ inexistant)

\section{Canal de largeur critique \\ Conal de faible largeur}

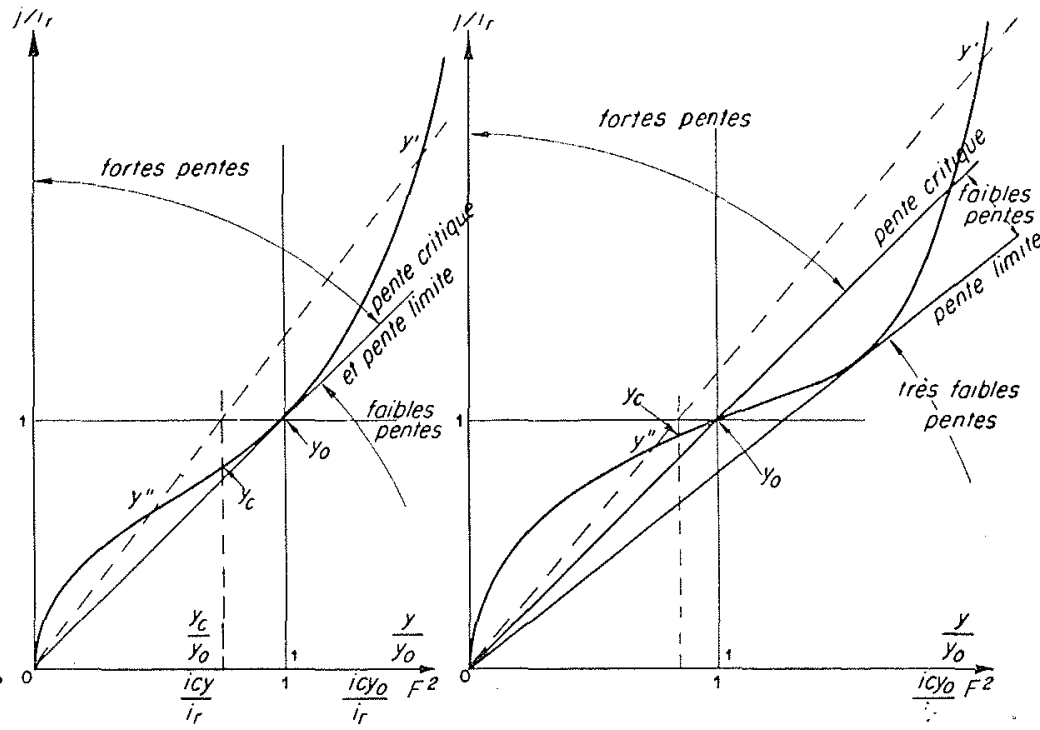

Pente forte..... $y^{\prime}<y_{0}<y_{c}<y^{\prime \prime}$

$\left.\begin{array}{l}\text { Pente limite } \\ \text { et pente critique : }\end{array}\right\} y_{c}=y_{0}=y^{\prime}=y^{\prime \prime}$

pente faible...... $y_{c}<y_{0}$

( $y^{\prime} y^{\prime \prime}$ inexistant)
Pente forte........ $y^{\prime}<y_{0}<y_{c}<y^{\prime \prime}$ Pente critique...... $y_{c}<y_{0}=y^{\prime}=y^{\prime \prime}$

Pente faible....... $y^{\prime}<y^{\prime \prime}<y_{c}<y_{0}$ Pente limite....... $y^{\prime}=y^{\prime \prime}<y_{c}<y_{0}$ Pente très faible. . $y_{c}<y_{0}$ 
canal; le canal circulaire est donc un canal de srande largeur pour $y_{0} / r<0,59$, un canal de faible largeur pour $y_{0} / r>0,59$ et un canal de largeur critique pour $y_{0} / r=0,59$.

Un canal trapézoüdal à parois latérales de forte pente aurait pour le radier deux pentes limites limitant trois zones dans lesquelles il $y$ a respectivement un point, trois points et un point a tangente horizontale; le mème canal à parois latérales de pentes moins fortes présente un seul point à tangente horizontale.

Le canal triangulaire s'identifie presque completement avec le canal rectangulaire de largeur infinie.

En résumé, l'auteur montre par son étude que les courbes de surface libre présentent bien théoriquement les points a tangentes horizontales comme l'avaient montré les auteurs cités plus haut. Il indique la cause de ces anomalies, la variation de $i_{c y}$, et classe ces courbes en fonction des differents critères qui les gouvernent.

L'imporiance de ces anomalies est donnée par la valeur de la différence $\Delta z$ entre deux points successifs à tangente horizontale (creux et sommet) ou entre un de ces points et l'asymptote ver's laquelle tend la ligne d'eau après ce point. La valeur de cette différence rapportéo à la profondeur normale $\frac{\Delta z}{y_{0}}$ est donnée par l'aire comprise entre la courbe de la fonction $\frac{d z}{d y}=f\left(\frac{y}{y_{0}}\right)$. M. Silber a effectué les calculs et obtenu les résultats suivants :

Canal infiniment large : $y_{0} / l=0$.

$$
\begin{array}{ll}
\frac{i_{r}}{i_{c y_{0}}}=1 \text { (pente critique) } & \frac{\Delta z}{y_{0}}=8 \% \\
\frac{i_{r}}{i_{c y_{0}}}=0,98 & \frac{\Delta z}{y_{0}}-6 \% \\
\frac{i_{r}}{i_{c y_{0}}}=0,95 & \frac{\Delta z}{y_{0}}=4 \%
\end{array}
$$

Le $\Delta z$ est relatif ici a l'écart entre le point à tangente horizontale et un point très éloigné $\left(\frac{y}{y_{0}}=3\right.$ environ $)$

Canal très large : $y_{0} / l=0,05$.

$$
\begin{array}{ll}
\frac{i_{o r}}{i_{c \eta_{0}}}=1 \text { (pente critique) } & \frac{\Delta z}{y_{0}}=4 \% \\
\frac{i_{r}}{i_{c y_{0}}}=0,98 & \frac{\Delta z}{y_{0}}=2,5 \% \\
\frac{i_{r}}{i_{c y_{0}}}=0.95 & \frac{\Delta z}{y_{0}}=1,2 \%
\end{array}
$$

$\Delta z$ n'est d'ailleurs pas ici la valeur entre le creux et le sommet, car ce dernier correspond a un $y / y_{0}$ très élevé, mais également entre le creux et le point $y / y_{0}=3$.

On peut déduire de cette étude que pour un canal rectangulaire, les écarts sont trop minimes pour pouvoir ètre réellement décelés; la forme classique du profil, tracé en négligeant le creux et le sommet, est donc pratiquement la forme de la ligne d'eau pour un canal de faible pente en régime fluvial. Par contre, dans un canal très étroit de forte pente, en régime fluvial, on pourrait observer pour une pente supérieure à la pente critique, une décroissance de la cote $z$ au lieu d'une croissance comme le donne la courbe classique. Il n'est d'ailleurs pas absolument exclu qu'avec certaines formes de section non usuelles, les phénomènes ne puissent être accusés et reudus decelables; le diagramme synthétique de M. Silber faciliterait, le cas échéant, l'étude critique de ces formes de section.

\section{Discussion}

M. le Président remercie M. Silibr d'avoir apporté par sa synthèse beaucoup de clayté dans les représentations des écoulements variés qui n'étaient connus jusqu'ici que fragmentairement.

M. Lazard, après avoir félicité M. Shlber de sa très intéressante et importante communication, signale qu“il avait modestement intitulé son travail de «Contribution à l'étude théorique du mouvement graduellement varié ».

Cependant, si M. Snber a étudié les formes des lignes d'eau en fonction de la pente du radier pour un débit donné, il parait intéressant de se placer à un autre point de vue et d'étudier la variation des écoulements en fonction du débit pour un radier donné.

C'est alors qu'apparaissent les notions de débit et de hauteur caractéristiques, extension des notions de MounET: le débit caractéristique est celui pour lequel la pente critique devient égale à la pente du radier. Cela exige que la pente du radier soit comprise dans un certain intervalle, qui dépend du profil du lit, et qui n'est nettement marqué que pour certains profils, par exemple le profil trapézoïdal.

Mais quel que soit le profil, cela entraine l'existence de lignes d'eau pratiquement horizontales, que l'auteur croit etre le premier à avoir signalées, et qui correspondent manifestement à la réalité : retenues des barrages par exemple.

Il est d'accord avec M. Sirmer pour reconnaître que les creux ou sommets qu'envisage la théorie sont très faibles, sans doute deux ou trois centimetres (plusieurs auteurs en ont mentionnes de tel ordre, mais en raison des difficultés de pareilles mesures, cela ne saurait être considéré comme une preuve de l'exactitude de la théorie).

M. LAzARD a calculé numériquement ce qui devrait se passer dans un canal trapézoüdal de pente 4 millièmes tel que le canal de fuite de Soulom s'il était suffisamment rectiligne. Pour les débits inférieur's au débit caractéristique le régime devrait être fluvial, pour devenir torrentiel pour les débits supérieurs.

Il montre les graphiques des lignes d'eau calculées : elles se ressemblent en fait tellement qu'il paraît douteux qu'une expérimentation ordinaire puisse mettre en évidence leurs différences théoriques.

An passage il souligne la grande importance pour les ealculs numériques du coefficient classique $\alpha$ des forces vives qu'il ne faut pas omettre dans les formules. Avec les valeurs extrêmes de 1 et 1,20 , les débits et hauteurs catractéristiques ont pris respectivement les valeurs de $7 \mathrm{~m}^{3} / \mathrm{s}$ et $90 \mathrm{~cm}$ et de $2 \mathrm{~m}^{3} / \mathrm{s}$ et $45 \mathrm{~cm}$.

Pour terminer, M. LAzARD pense qu'il y aurait probablement intérêt (économique), quand les circonstances s'y prêtent, à déterminer la pente du radier d'un canal de profil donné de telle façon que le débit maximum a écouler soit voisin du débit caractéristique correspondant.

M. Silber reconnaît que M. Lazard envisage le même problème que lui mais d'un autre point de vue : l'étude de la variation de l'expression $\frac{i_{r}}{i_{c y_{0}}}$ lorsque $Q$ varie en entrainant la variation de $y_{0}$. Il s'agit de voir comment on évolue dans le diagramme en faisant varier ces paramètres.

En ce qui concerne les variations de $\alpha$, M. Silber répond qu'il suffit, pour en tenir compte, d'introduire dans l'expression du nombre de Froupe la valeur $\alpha \frac{U^{2}}{2 g}$ à la place de $\frac{U^{2}}{2 g}$. Dans ces conditions, le dia- 
gramme est vrai une fois pour toutes et seule la graduation en $\frac{y}{y_{0}}$ est influencée faiblement par les faibles variations de $\alpha$.

Après une suspension de séance de quelques minutes, la parole est donnée à M. MArtre, ingénieur au Laboratoire National d'Hydraulique, pour sa communication:

\section{NOTE SUR LES DÉPRESSIONS, AUX OUVERTURES PARTIELLES DES VANNES, DANS LES ÉVACUATEURS DE CRUE ET LEUR RÉPERCUSSION SUR LE CHOIX DU PROFIL DÉVERSANT ET DE LA POSITION DES VANNES.}

Le mémoire de M. Martre et la discussion seront publiés dans le $\mathrm{n}^{\circ} 2-1952$ de la Houlle Blanche.

M. Radiguer prend la présidence de la séance pendant une courte absence de M. GanisL et donne la parole à M. Escande.

\section{COMMUNICATION DE M. ESCANDE}

\section{ETUDE DE LA STABILITÉ DES CHAMBRES D'ÉQUILIBRE A ÉTRANGLEMENT}

\section{Résumé}

\section{Premiéne partie}

En ce qui concerne les chambres d'équilibre ordinaires, sans étranglement, depuis l'énoncé de la condition fondamentale de Thoma en 1910, de nombreux auteurs (en particulier Schuller, Karas, Frank, JaEger, Foncheimer,

$$
\begin{aligned}
& - \text { pour } m<r_{1}=\frac{1-2 p_{0} h_{0}}{h_{0}-2 p_{0}} \ldots \ldots \ldots \\
& \text { - pour } m=r_{1} \ldots \ldots \ldots \ldots \ldots \\
& \text { - pour } r_{1}<m<r_{2}=r_{1}+\frac{2\left(h_{0}-1\right)}{h_{0}-2 p_{0}} \\
& \text { - pour } m>r_{2} \ldots \ldots \ldots \ldots \ldots \ldots \ldots
\end{aligned}
$$

$2^{\circ}$ En tenant compte de la loi de variation réelle de la perte de charge $r=r_{0} v^{2}$, nous arrivons par des considérations d'équivalence énergétique, à déduire de la théorie précédente des résultats généraux applicables aux problèmes de l'industrie.

Dans une chambre d'équilibre à étranglement, de section inférieure à la section limite de THOMA, il s'établit, dans tous les cas, autour du niveau de régime permanent de débit $Q_{0}$, un régime stable d'oscillations entretenues.

Dans l'hypothèse d'une variation sinusoïdale. de ", l'expression approchée :

$$
v_{\mathrm{M}}=1,18 \frac{r_{1}}{r_{0}}=\frac{1,18}{r_{0}} \cdot \frac{1-2 p_{0} h_{0}}{h_{0}-2 p_{0}}
$$

donne l'ordre de grandeur de l'amplitude $v_{\mathrm{X}}$ des oscillations entretenues correspondantes de la vitesse $v$.
Franirovic, Cuenod, Gardel, Boner et tout spécialement GADEN) ont apporté de précieuses contributions au problème fondamental de la stabilité.

Pour les chambres d'équilibre à étranglement, il n'y a pas eu, à notre connaissance du moins, d'études précises de leur stabilité.

Citons toutefois la conclusion donnée par Calame ef GADen, dans leur ouvrage classique, à leur analyse qualitative des conditions de fonctionnement des chambres a étranglement :

«Une chambre à étranglement peut donc éventuellement, si la section est insuffisante, devenir le siège d'oscillations qui vont en s'amplifiant pour de petites amplitudes, puis d'oscillations entretenues et enfin amorties, mais pour de plus grandes amplitudes seulement.»

Les travaux que nous résumons dans cette brève communication montrent tout le bien fondé de cette conclusion.

\section{DeUxiène parte}

$1^{\circ}$ En faisant l'hypothèse d'une perte de charge à la traversée de l'étranglement proportiomelle à la vitesse $r=m v$, nous arrivons, par un raisomement analogue à celui qui conduit Troma à sa condition classique, a l'équation différenticlle des oscillations entre grandeurs relatives :

$$
\begin{aligned}
\left(h_{0}-m\right) \frac{d^{2} x}{d t^{\prime 2}} & +2 \pi\left[2 p_{0} h_{0}-1+m\left(h_{0}-2 p_{0}\right)\right] \frac{d x}{d t^{\prime}} \\
& +4 \pi^{2}\left(h_{0}-2 p_{0}\right) x=0
\end{aligned}
$$

Dès lors, si nous nous limitons à l'étude des chambres d'équilibre ne satisfaisant pas a la condition de Thoma $\left(2 p_{0} h_{0}<1\right)$ et si nous supposons remplies les conditions :

$$
h_{0}>1 \quad h_{0}-m>0 \quad h_{0}-2 p_{0}>0
$$

tonjours vérifiées en pratique, nous aboutissons aux conclusions suivantes:

oscillations indéfiniment amplifiées.

oscillations entretenues de période : $\quad \theta=\frac{\sqrt{h_{0}^{2}-1}}{h_{0}-2 p_{0}}$

oscillations amorties.

amortissement sans oscillations.

$3^{\circ}$ Nous rappelons que nous avons indiqué à la page 256 de notre ouvrage Recherches sur les chambres d'équilibre (Gauthier-Villaxs, 1943), en lui apportant une rectification de détail à la page 76 de notre ouvrage Méthodes nouvelles pour le calcul des chambres d'équilibre (Dunod, 1950), une méthode graphicue applicable à l'étude des oscillations dans une chambre d'équilibre à étranglement.

L'étude systématique, au moyen de cette méthode graphique, de 44 exemples, pous a permis de vérifier la parfaite exactitude du résultat concernant l'existence, dans tous les cas, d'un régime stable d'oscillations entretenues.

Ces oscillations ne sont pas rígoureusement, sinusoïdales, ce qui mous conduit à poser :

$$
x_{1 \mathrm{M}}=-k_{1} \frac{r_{1}}{r_{0}} \quad x_{2 \mathrm{M}}=k_{2} \frac{r_{1}}{r_{0}}
$$




$$
v_{1 \mathrm{ML}}=-k_{1} \frac{r_{1}}{r_{6}} \quad v_{2 \mathrm{M}}=k_{2} \frac{r_{1}}{r_{0}}
$$

$v_{1 \mathrm{M}}, v_{2 \mathrm{I}}, x_{1 \mathrm{M}}, x_{2 \mathrm{M}}$ désignant les amplitudes maxima des alternances positives et négatives de $v$ et de $x$, grandeur relative de la cole instantanée du plan d'ean audessus du niveau dynamique de régrime permanent considèré.

L'étude graphique conduit alors aux résultats ci-apres :

$1^{\circ}$ Pour les valeurs de $r_{0}$ supérieures à $1,30 r_{1}$, valeurs qui sont seules à considérer en pratique, puisque leur limite inférieure $\left(r_{0}=1,30 r_{1}\right)$ donne à l'expression $1,18 r_{1} / r_{0} . Z_{x}$ une valeur $0,90 Z_{x}$ inadmissible en pratique, les coefficients $k_{1}$ et $l_{2}$ restent supérieurs à l'unité.

$2^{\circ}$ Les coefficients $k_{1}^{\prime}$ et $k_{2}^{\prime}$ ne dépassent guère pratiquement 1,18 . Le plus souvent, ils sont inférieurs à 1,18 et l'écart correspondant augmente quand $h_{0}$ diminue.

D'autre part, il suffit que $r_{0}$ soit supérieur ì $1,10 r_{1}$ pour que $k_{1}^{\prime}$ et $k_{2}^{\prime}$ soient supérieurs à l'unité.

$3^{\circ}$ Les valeurs de $k_{1}$ et $k_{2}$ sont pratiquement confondues pour $p_{0}=0$, et il en est de même des valeurs de $k_{1}^{\prime}$ et $k_{2}^{\prime}$.

Quand $p_{0}$ n'est pas nul, $k_{1}$ et $k_{1}^{\prime}$ deviemnent respectivement supérieurs à $k_{2}$ et $k_{2}^{\prime}$, la différence $k_{1}-k_{2}$ étant nettement supérieure à $k_{1}^{\prime}-k_{2}^{\prime}$.

Nous pouvons done conclure cette seconde partie de la manière suivante :

Pour toutes les oscillations entretenues susceptibles d'être envisagées dans des cas pratiques, l'expression:

$$
x_{\mathrm{M}}=1,18 \cdot \frac{r_{1}}{r_{0}} Z_{\odot}
$$

fournit, avec une approximation souvent excellente et toujours suffisante, l'amplitude de ces oscillations, l'erreur commise demeurant inferieure it $15 \%$ et restant toujours dans le sens de la sécurité.

\section{TroISIEMAE PARTIE}

En pratique, certains éléments que néglige la théorie interviennent pour améliorer la stabilité du fonctionnement des chambres d'équilibre.

Tout d'abord, la marche en parallèle constitue un élément majeur : comme l'ont montré Calane et Gaden, si la puissance de l'installation considérée est inférieure au tiers de la puissance totale du réseau, le régime de marche est stable, quelle que soit la section de la chambre d'équilibre.

Même dans le cas de la marche isolée sur réseau séparé, la stabilité semble être plus grande que l'indiquent les conditions théoriques : citons, à cet égard, les expériences italiennes de GheTt, Evangeristi et tout. particulièrement Schiemi qui, à l'usine de Fadallo, a mis en évidence l'amortissement des oscillations dans une chambre d'équilibre ordinaire de section inférieure à la moitié de la valeur limite donnée par la condition de Troma.

Des dispositifs très intéressants permettent, d'autre part, d'éliminer les oscillations, par asservissement temporaire de la puissance électrique à la pression hydraulique : citons, à ce sujet, l'étude de MM. Curvod et Garder parue dans le Bulletin Technique de la Suisse Romande du 12 août 1950 , et le remarquable travail que MM. GADEN et Borer ont fait paraitre dans le Bulletin Technique de la Suisse Romande du 4 mai 1951.

\section{QuATRIÈE PARTE}

Il résulte des considérations précédentes que, pour les chambres d'équilibre ordinaires, il n'est pas toujours néeessatie de respecter de laçon tout à fait rigoureuse la condition de THome, mêne si l'usine doit fonctionner partois à réseau séparé.

En particulier, dans le cas des chambres d'équilibre a etranglement, on peul, comme nous l'avons montré, réduire la section à une valeur neftement inferreure à la limite de Thoma.

Les oscillations entretenues, à amplitude réduite, dont la théorie permet de prévoir l'existence, semblent devoir ètre parfois éliminées par les facteurs d'amortissement dont ne tiemment pas compte les calculs théoriques.

Dans le cas où ces oscillations subsisteraient, il serait facile de les éliminer par l'adoption d'un dispositif d'asservissement temporaire de la puissance électrique à la puissance hydraulique. L'emploi d'un tel dispositif, pour une chambre d'équilibre ordinaire, peut faire naître la crainte des conséquences graves (déversements ou entrées d'air dans les conduites) que pourrait entrained un non-fonctionnement du système. Ces craintes ne peuvent plus exister pour une chambre a etranglement, convenablement dimensionnée, puisque le non-fonctionnement momentané de l'asservissement n'aurait d'autre conséquence que de laisser subsister des oscillations entretenues, d'amplitude restreinte et sans aucune conséquence fàcheuse.

\section{Discussion}

M. le Président Gariel reprend la présidence, remercie M. Escande, rappelle que M. DE SPARRE avait indiqué vers 1917 que l'adjonction d'un étranglement à la base de la cheminée pourrait conduire à un très intéressant amortissement des oscillations.

M. RÉmb́ntéras est heureux de trouver dans le travail de M. Escande un argument pour justifier l'opinion qu'il a déjà exprimée, suivant laquelle on peut dans de nombreux cas ne pas respecter strictement la condition de 'Tном. Certes, jusqu'ici on pouvait toujours étudier la stabilité d'une cheminée de type et de forme quelconques par des méthodes analytiques ou graphiques ainsi que M. Escande l'a montré dans un travail antérieur. On pouvait encore exécuter à cet effet un essai sur modèle réduit; grâce à d’ingénieux artifices imaginés par M. Danel, on pouvait ainsi tenir compte de la variation du rendement de la turbine avec la chute, de l'influence des pertes de charge, etc., tous éIéments qu'il est difficile d'introduire dans des calculs de stabilité. Mais ces méthodes longues et coûteuses ne se justifient que pour des ouvages importants. Il faut remercier M. Escande d'apporter aux ingénieurs des bureaux d'études une méthode rapide pour se rendre compte des mouvements qui pourraient résulter de la non-observance de la condition de Thoma.

Toutefois il faut observer que, sauf dans le cas des chutes assez basses à gros débit, il est rave que la condition de Thoma soit « déterminante » pour le dimensionnement de la cheminée d'équilibre. D'autres considérations imposent à l'ouvrage un volume plus ou moins étroitement fixé. M. Gader a montré qu'en l'absence d'étranglement ce volume serait minimum pour une cheminée comportant un puits «filiforme , muni à ses deux extrémités de chambres d'expansion de grande surface et de faible hauteur. En pratique il sera parfois plus économique - malgré une augmentation a volume total de l'ouvrage - de supprimer ou de réduire les chambres d'expansion en augmentant le diamètre du puits qui satisfera souvent largement is la condition de THoma.

M. Escinde est d'accord aveć M. Rúmóntrisas quant au fait que ce n'est pas la condition de Thoma qui limite en général la section et cite deux exemples: l'usine de Saint-Christau, sur la Garonne, et l'usine de .Tablanica en Yougoslavic. 
M. RÉmíñas ajoute que, de toute façon, il est généralement économique d'adopter une cheminée á étranglement et si, suivant le système préconise par M. Escaros, on choisit un diamètre de puits qui peut entrânet une oscillation entretenue du plan d'eau dans la cheminée, il serait interessant d'avoir lavis des constructeurs de turbines et de régulateurs sur l'amplitude relative (c'esta-dire par rapport à la hauteur de chute) maximum tolérable pour cette oscillation.

M. le Président Ganel précise son point de vue sur cette question : ces oscillations, meme si elles ont des périodes de 5 à 10 minutes, ne sont pas a priori « mortelles », mais les maitres de l'cuvre ont jusqu'à présent estimé qu'elles étaicnt inopportunes car elles pouvaient produire, à la longue, certaines usures telles que celle des distributeus.

M. Escande souligne qu'à dépense égale, la forme à section uniforme et à etranglement est plus intéressante du fait que la contre-pression au départ est produite, non par la hauteur statique mais par la perte de charge a travers le rétrécissement et que celle-ci est d'autant plits grande que l'étranglement est plus important.

M. Rémériéras observe enfin que tandis que M. EsGanes a donné la condition d'amortissement de grandes oscillations, la condition de Thoma dans sa forme rigoureuse indique que, pour des vitesses d'oseillations très petites, le rétrécissement n'intervient pas, la tangente à la courbe de perte de charge en fonction de la vitesse de cet organe étant de toute façon nulle ou très faible atu voisinage de l'origine.

M. Escande répond que l'oseillation s'amplifie alors et que la vitesse vient automatiquement se placer en un point tel que la tangente it la conrbe intervient pour réaliser l'effet du rétrécissement et limiter l'amplification de l'oscillation. Au demeurant, ces oscillations d'amplitude restreinte ne se produiront que dans la marche sur réseau séparé, et en cas de panne da dispositif d'asservissement.

La discussion ne pouvant être épuisée vu l'heure, M. le Président rappelle que les membres peubent envolyer des observations par écrit au Secrétariat de la S.H.F. après la séance.

Répondant à cette invitation, M. Bouvaro nous a fait parvenir une note dont nous reproduisons ci-après les conclusions :

Dans la plupart des galeries d'amenéc à cheminéc d'équilibre à étranglement optimum, la condifion de Thoma sera satisfaite si l'ouvrage est calculé pour assurer une fermeture complète instantanée.

Il a été demandé à $M$. Bovvano d'exposer en détail son point de vue à une prochaine réunion.

M. Ie Président donne la parole à M. Branchex pour sa communication :

\section{LES SIPHONS DE DËCHARGE DANS LES CHAMBRES D'EAU}

Le mémoire de M. BLANCHET et la discussion qui a suivi sa présentation en séance ont été publies page 648. du $n^{\circ}$ B-1951.

La séance est levée à 19 h 10 .

\section{SÉANCE DU MERCREDI MATIN 20 JUIN 1951}

La séance est onverte à 9 h 15 sous la présidence de M. HUPNER.

Après l'adoption du procès-verbal de la session précédente et la présentation du deuxième volume des Sláions Hydrométriques Francaises édité par la S.H.F. pour la région du Massif Central, M. le Président annonce la communication de M. MoRIAT :

\section{NOTE SUR L'ÉVALUATION DU DÉBIT DE CRUE}

et donne une brève analyse de cette communication en vue de la discussion qui suivra sa présentation.

Le mémoire de M. Monlat et la discussion ont été publiés pages 663 et suivantes du n $\mathrm{B}-1951$.

\section{NOTES DE M. COUTAGNE}

\author{
PRÉSENTÉES PAR M. VANTROYS
}

M. VANrnoys, en s'excusant de présenter ces notes au pied levé à la place de M. Gibrat, empêché, donne un apercu intéressant des applications du calcul des probabilités aux houles journalières en vue des projets d'usines marémotrices : il a installé un cnregistreur de houles qui fonctionne depuis deux ans et dont le déponillement des observations devra être fait d'une manière analogue au dépouillement des observations de crue. La difficulté actuelle pour la houle vient du manque total d'observations dans le passé.

\section{1" VARTABILITE DES DEBITS DES COURS D'EAU D'APRES LANE ET KA I LEI}

\section{Résumé}

Un index de variabilité des cours d'eau est proposé sous forme de l'écart-type du logarithme des débits. La méthode de détermination de cet index et les valeurs qu'il prend pour de nombreux con's d'eau orientaux des Elats-Unis sont indiquées. L'emploi de cet index dans l'évaluation de la courbe de durée (1) du cours d'eau (lorsque le nombre d'observations est réduit ou inexistant) est exposé.

Une étude des index ainsi que des précipitations respectives a montré que dans la partie nord-est des EtatsUnis, la nature du sol (y compris sa couverture végétale) et la présence de lacs, de marais, sont les facteurs les plus importants dans la variabilité des débits pendant la plus grande partie du temps et que les autres facteurs ont relativement peu d'effet.

Ce compte rendu est un condensé d'une étude plus détaillée déposée à la Bibliothèque des Sociétés d'Ingénieurs (2) oì elle peut être examinée ou dont des copies ou microfilms peuvent être obtenus.

\section{Discussion}

Sur la demande de M. le Présidint Barridon, M. VanTroys précise que la méthode de Lane et KaI Lei fait

(1) Débits classés.

(2) Adresse : 29 W. 39 th St., N.X. $18 \mathrm{~N} . \mathrm{X}$. 
intervenir le logarithme du débit journalier et se rattache ainsi à la méthode Grinat par la relation ci-après cntre le coefficient $a$ de Grusat et l'index de variabilite $i$ de LANE et KaI LEI :

$$
a=\frac{\sqrt{2}}{2 i}
$$

M. le Président voudrait savoir si les Américains ont eu des cas de grande variabilité des débits annuels analogues à ceux de certains oueds tunisiens où le débit a varié parfois dans le rapport de 1 à 180 suivant les années; M. Vantroys répond que les index ciés par lane et KAI Ler ne varient pas dans de grandes limites.

\section{$2^{\circ}$ COMMENTAIRES RECENTS SUR LES TRAVAUX} $D E M$. GUMBEL

M. Coutacine a traduit un certain nombre de commentaires publiés dans la presse des Etats-Unis d'Amérique sur les travaux de Gumber.

Ces publications sont :

B. F. Kmball. - Type réduit de distribution des probabilités primaires appliqué aux écoulements annuels maxima de crue. (The Annals of Math. Statistics, vol. $13, \mathrm{n}^{\circ} 3,1942$.)

Ralphe W. Powerz. - Une méthode simple d'évaluation de la fréquence des crues. (Civil Engineering, vol. $13, \mathrm{n}^{\circ} 2,1943$.)

Reginald C. Price. - La méthode de Gumbel d'estimation de la fréquence des crues. (Civil Engineering, vol. $13, \mathrm{n}^{\circ} 6,1943$.)

William P. Cross. - Crues de l'Ohio, grandeur et fréquence. (Balletin $n^{\circ} 7$, Ohio W.R.B.)

'T. Dairymple. - L'emploi des statistiques de débits dans l'étude des chenals de ponts. (Proceedings, décembre 1946.)

Will Lrssnen. - N. Y. Times, January 26, 1947.

S. B. Litrauen. - Bulletin of the Inst. of Experimentai Methods, vol. I, n' $4,1947$.

Manuel de l'Hydrologiste. - Geological Survey, U. S. Department of the Interior, April 1947.

C. J. VELz. - Factors influencing self purification and their reiation to pollution abatement. (Sewage Works Journal, vol. 19, no 4, 1947.)

Floods in Columbia river basin (Water Supply Paper, pp. 326-328.)

N. Y. Times, 10 avril 1949. - New calculation used to cut error.

N. Y. Times, 19 juin 1949. Predicting floods by a new method.

Tous ces articles donnent soit un exposé succinct de la méthode de la loi statistique de la plus grande valeur de Gunrel et de son application aux débits des cours d'eau, bien connue des hydrologues, soit des commentaires sur son utilité et lexcellence de son ajustement aux observations des cours d'eau américains.

Parmi ces commentaires, on peut citer notamment :

« Considération du facteur temps:

«Gummer ne cherche pas à déterminer quelle est la plus grande crue susceptible de survenir dans un bassin. Une crue double de la crue la plus forte observee peut ne pas se produire avant mille ans ou, au contraire, survenir l'année prochaine. Statistiquement parlant, il n'y a pas de crue maximum possible, mais il existe un facteur temps pendant lequel une crue d'un volume donné peut survenir : Gumber, par conséquent, considère l'élément temps.

« Soit nin barrage ou un pont qui doit durer deux siècles. D'après Gumber, le statisticien détermine quelle doit être la plus grande crue qui se produira dans cette période.

«Gumbe introduit le concept de la période de récurrence, c'est-à-dire le temps qu'il faut pour qu'un événement de caractéristiques données se reproduise en moyenne une fois. Imaginons que la période de récurrence de la plus grande crue observée en 50 ans soit de l'ordre de 2.500 ans. Il serait ridicule de construire un pont ou un barrage devant résister à une crue double de la plus grande qui s'est produite en cinquante ans.

"Les applications en sont nombreuses (prédiction des plus grosses averses, des plus sévères sécheresses, des températures maxima et minima, des sccousses sismiques), toutes fonctions du temps.»

(New York Times, 19 juin 1949.)

\section{Discussion}

M. Vantroxs signale la note rédigée par M. Gmrat pour exposer à la Commission d'étude des débits de crue la comparaison entre ses propres travaux et ceux de Gumbel :

Dans le cas particulier auquel il faisait allusion avant de commencer la lecture des notes de M. Covtagne qui est l'étude de la probabilité de la houle en un certain point, M. VANTroys croit que la méthode des maxima, qui est celle de M. Gunmer, est plus directement applicable que la méthode des enregistrements permanents.

M. Barrillon rappelle qu'un certain nombre d'exemplaires de cette note ont été distribués à la dernière réunion de la Commission; quelques tirages sont encore disponibles et seront envoyés aux membres intéressés qui en feront la demande au secrétariat.

M. Barrillon demande que ces membres nous adresscnt leurs remarques éventuelles sur cette note; la publication dans «Mémoires et Travaux » de la note complétée par les remarques des commentateurs pourra, ensuite, être proposée au Comité de rédaction, après accord avec M. Gibrat, en vue notamment de remplacer un mémoire de M. Gmnat édité en 1936 par la S.H.F, et dont le stock est actuellement épuisé.

M. le Président remercie M. Vantroys.

\section{ESSAI D'ÉTUdE STATISTIQUE DES DÉBITS DU RHIN A STRASBOURG (1881-1951) COMMUNIQUÉS PAR LE SERVICE DES PONTS ET CHAUSSÉES A STRASBOURG}

\author{
par M. BOYER
}

\section{Résumé}

* La station de Strasbourg, située au centre d'un secteur du fleuve dont le régime varie peu entre Bâle et l'embouchure du Neckar, a toujours été une des stations principales du Rhin.

« Les études basées sur l'échelle de Strasbourg exigent toutefois des précautions spéciales, du fait de la dérivation du Rhin à cet endroit par le Petit-Rhin et par le canal de l'Electricité de Kehl, et par suite aussi des mouvements généraux du lit, particulièrement importants dans cette partie du Rhin où le profil en long n'a pas encore trouvé son équilibre... Un grand nombre de jaugeages ont été effectués depuis de nombreuses années, et pour comparer une observation ancienne avec une obser- 
vation actuelle, il faut, au préalable, corriger l'observation ancienne, pour tenir compte des variations des niveaux qui résultent des modifications générales du lit... C'est ainsi qu'on a calculé les moyennes des niveaux mensuels moyens maxima et minima ainsi que les maxima des niveaux mensuels maxima et les minima des niveaux mensuels minima pour la période de 70 années $1881-1950$.

«Le caractère prédominant de ce régime est celui des fleuves alpestres : le Rhin ne subit pas encore a Strasbourg une influence notable des afluents de plaine. D'autre part, le régime du Rhin est relativement régulier par suite de l'importance des lacs existant dans son bassin. Le bassin versant du Rhin a Strasbourg a une superficie de $39.600 \mathrm{~km} 2$ environ, dont $1.240 \mathrm{~km}^{2}$ de lacs et $660 \mathrm{~km} 2$ de glaciers. La période de basses eaux se produit pendant les mois de décembre à mars, avec l'étiage en février et la période de hautes eaux pendant les mois de juin et juillet; les plus fortes crues se preduisent en juin, septembre et décembre, causées en généjal par une période de fortes pluies coüncidant avec une elévation de la température amenant une fonte des neiges.

"Le plus bas niveau observé pendant la période étudiée a été réalisé le 3 novembre 1947 avec $0,60 \mathrm{~m}$. Antérieurement on avait observé la cote $0,98 \mathrm{~m}$ le 11 janvier 1894 , ce qui correspond aujourd'hui à la cote $0,52 \mathrm{~m}$. Plus récemment, on a observé, le $27 \mathrm{mal}^{\prime} \mathrm{s} 1921$, la cote $0,84 \mathrm{~m}$ qui correspond aujourd'hui à la cote $0,55 \mathrm{~m}$. Le plus haut niveau observé pendant la même période a été

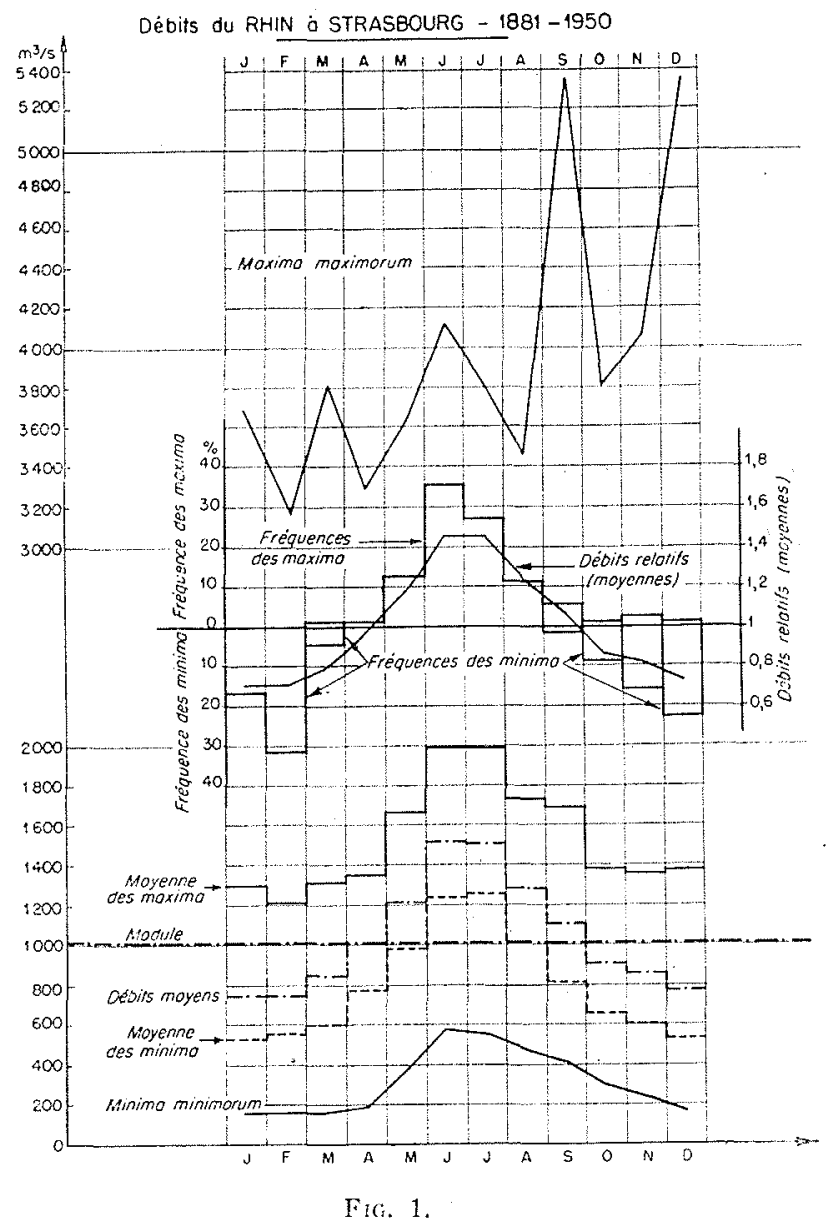

Données de M. Portrat,

Ingénieur des Ponts èt Chaussées

à Strasbourg réalisé le 4 septembre 1881 et le 28 décembre 1882 avec la cote $6,47 \mathrm{~m}$, qui correspond aujourd'hui à la cote $6,41 \mathrm{~m}$; mais on a observé, le 15 juin 1876 , un niveau quelque peu supérieur avec lá cote $6,65 \mathrm{~m}$ qui correspond aujourd'hui à la cote $6,55 \mathrm{~m}$ environ. La dénivellation maximum atteint done $6 \mathrm{~m}$ environ. 》

\section{R. Porthat.}

Ces lignes, extraites des commentaires de M. Portrat (Strasbourg, juin 1951), situent les domées de l'étude de M. BOYER.

Le caractère du Rhin tel qu'il est décrit ci-dessus est schématisé par les graphiques de la figure 1.

Les résultats de l'étude statistique sont condensés et confrontés avec les résultats de $M$. Coutracine pour le Rhin à Bâle et la Loire à Montjean (1) dans le tableau ci-après (page 133); ces résultats sont, d'autre part, représentés par les graphiques des figures suivantes:

- Fig. 2. - Courbe des fréquences par classes (histogramme) et courbe des débits classés.

- Fig. 3. - Variabilité du module.

- Fig. 4. - Variabilité du débit de crue.

-.. Fig. 5. - Crues probables suivant diverses lois en fonction de la période de retour.

Variabilité du module. - On voit sur la figure 3 que les points observés se situent sensiblement entre les deux droites de Gauss pour les probabilités comprises entre $20 \%$ et $95 \%$, mais que la courbe déduite de la loi de Gatton suit mienx la courbe des valeurs observees, notamment pour les valeurs faibles et moyennes de la distribution.

Cependant, le plus bas et le plus fort modules centenaires (probabilités $1 \%$ et $99 \%$ ) semient respectivement :

$1.516 \mathrm{~m}^{3} / \mathrm{s}$ et $576 \mathrm{~m}^{3} / \mathrm{s}$ suivant la loi de Gauss;

$1.639 \mathrm{~m}^{3} / \mathrm{s}$ et $879 \mathrm{~m}^{3} / \mathrm{s}$ suivant la loi de Galton.

Les plus forts et les plus faibles modules des 70 années d'observations sont respectivement :

$1.47 \mathrm{~m} 3 / \mathrm{s}$ en 1910 , et $537 \mathrm{~m} 3 / \mathrm{s}$ en 1921 .

La loi de Gauss serait donc plus fidèle que la loi de Galton pour ces deux valeurs extrêmes, qu'elle donne avec un excès de 5 à $7 \%$.

Variabilité du débit de crue (fig. 4 et 5). -.- Lat loi de Gauss interprète aussi fidèlement que toute autre formule la distribution statistique du débit de crue entre les probabilités $5 \%$ et $95 \%$; mais la formule de Gauss ne peut être extrapolée an-delá de ces limites.

Les courbes de Gaton et de Gumber-Fulder constituent des tangentes à la courbe de Gauss, à laquelle elles se substituent à partir d'une certaine probabilité.

Cependant, le domaine des observations (intervalle de confiance) nous parait trop restreint pour en tirer des extrapolations indiscutables.

Corrélation hydrométéorologique. - Enfin, la corrélation des débits mensuels moyens avec la moyenne des précipitations observées à dix stations suisses du bassin rhénan est esquissée par le diagramme de la figure 6 , qui montre un certain parallélisme, nuancé toutefois en automne, sans doute par l'influence des pluies sur les affluents de plaine.

En considérant seulement les modules des dix années

(1) D'aptès les données de MM. Pandé ct Ginert (La Honille Blanche, no A-1951, p. 287.) 


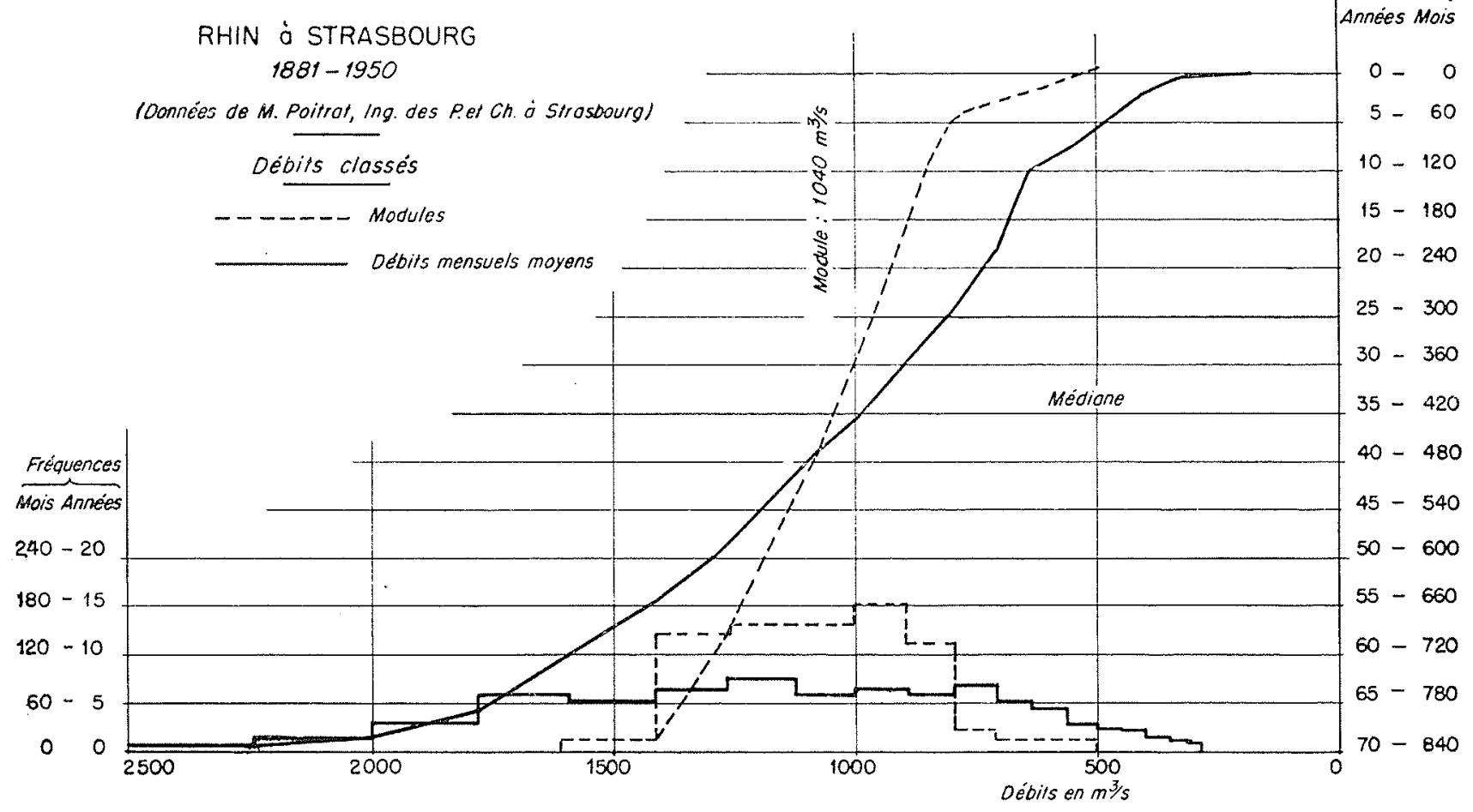

lig. 2.

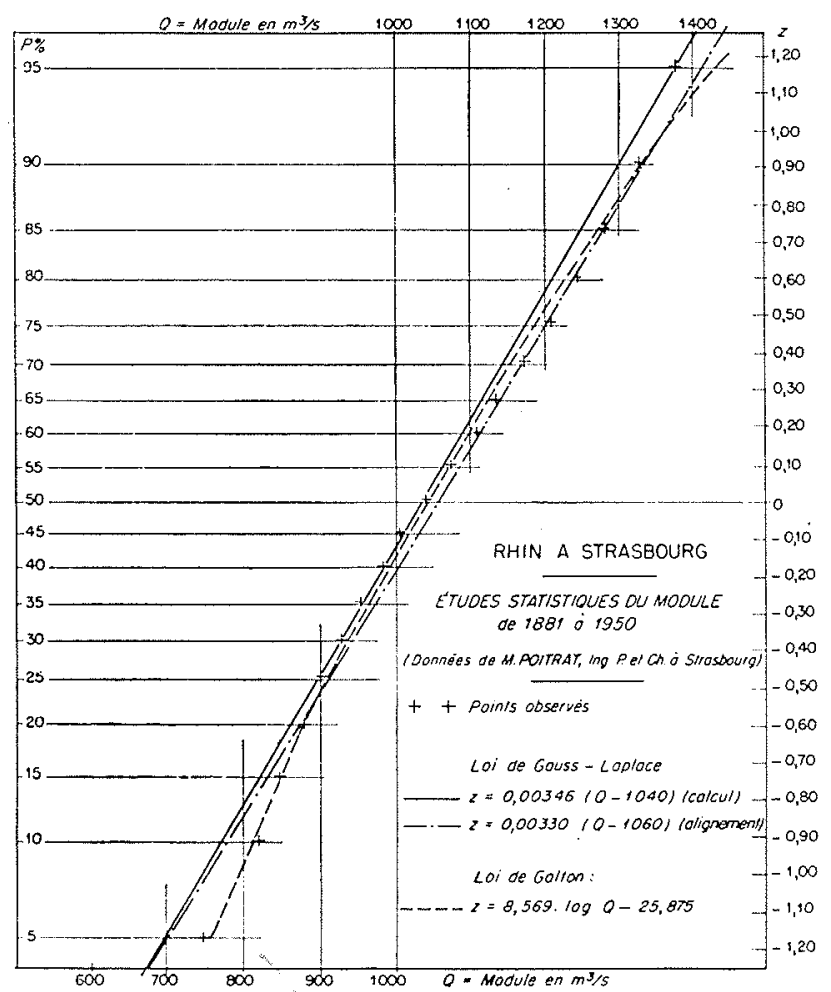

Frg. 3.

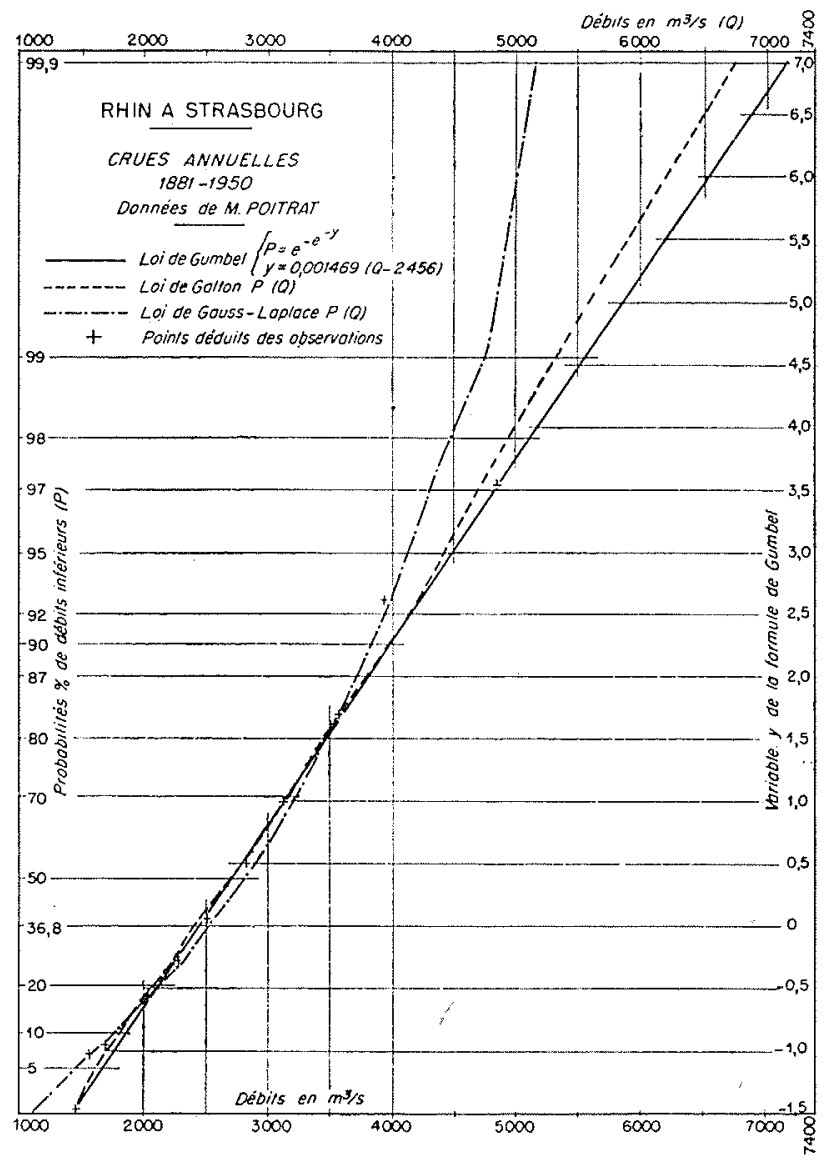

Fici. 4. 


\begin{tabular}{|c|c|c|c|}
\hline & $\begin{array}{c}\text { RHIN a STRASBOURG } \\
1881-1950\end{array}$ & $\begin{array}{c}\text { RHIN A BALE } \\
1808-19+7\end{array}$ & $\begin{array}{c}\text { LOME A MONTJEAN } \\
1880-1949\end{array}$ \\
\hline & \multicolumn{2}{|c|}{ DEBIT MOYEN ANNUEL } & \\
\hline Module & $1.0+0 \mathrm{~m} 3 / \mathrm{s}$ & $1.025 \mathrm{~m} 3 / \mathrm{s}$ & $773 \mathrm{~m} 3 / \mathrm{s}$ \\
\hline Débit médian $\ldots \ldots \ldots \ldots \ldots \ldots$ & $1.040 \mathrm{~m}^{3} / \mathrm{s}$ & $1.005 \mathrm{~m}^{3} / \mathrm{s}$ & $773 \mathrm{~m}^{3} / \mathrm{s}$ \\
\hline Débit dominant $\ldots \ldots \ldots \ldots \ldots \ldots$ & & & \\
\hline $\begin{array}{l}\text { Variabilité saisonnière (écart } \\
\text { moyens des débits mensuels) .. }\end{array}$ & $900<Q<\underset{0,231}{<1.000 \mathrm{~m}^{3} / \mathrm{s}}$ & 0,27 & 0,51 \\
\hline Rapport des extrèmes ......... & 2,74 & 2,36 & 2,20 \\
\hline $\begin{array}{l}\text { Coefficient de variation (rapport } \\
\text { ecart type/module) } \ldots . . \ldots \ldots \ldots\end{array}$ & 0,192 & 0,16 & 0,36 \\
\hline 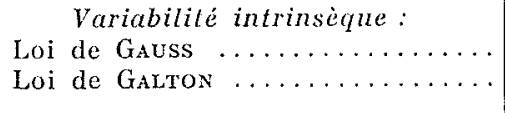 & $\begin{array}{c}Q=1.040+289 z \\
z=8,57 \log (0 / 1.046)\end{array}$ & $\begin{array}{c}Q=1.025+230 z \\
z=10.7 \log (Q / 1.014)\end{array}$ & $\begin{array}{c}Q=843+446 z \\
z=4,30 \log (Q / 777)\end{array}$ \\
\hline & DËBIT MOYEI & N MENSUEL & \\
\hline Débit médian observé ...... & $980 \mathrm{~m}^{3} / \mathrm{s}$ & & \\
\hline $\begin{array}{l}\text { Variabilité intrinsèque } \\
\text { Loi de Gauss } \ldots \ldots \ldots \ldots \ldots \ldots \ldots \ldots \ldots \ldots \\
\text { Loi de Galtos } \ldots \ldots \ldots \ldots \ldots \ldots\end{array}$ & $\log Q=3+0,287 z$ & $\begin{array}{c}Q=1.015,6+600 z \\
\log Q=2,97+0,266 z\end{array}$ & $\log Q=2.757+0,571 z$ \\
\hline & DEBIT DI & E CRUE & \\
\hline Débit moyen $\ldots \ldots \ldots \ldots \ldots \ldots$ & $2.829 \mathrm{~m}^{3} / \mathrm{s}$ & $2.765 \mathrm{~m} 3 / \mathrm{s}$ & $3.564,2 \mathrm{~m}^{3} / \mathrm{s}$ \\
\hline Débit médian $\ldots \ldots \ldots \ldots \ldots \ldots$ & $2.697,5 \mathrm{~m}^{3} / \mathrm{s}$ & $2.692 \mathrm{~m}^{3} / \mathrm{s}$ & $3.627,5 \mathrm{~m}^{3} / \mathrm{s}$ \\
\hline Débit dominant $\ldots \ldots \ldots \ldots \ldots$ & $2.456,2 \mathrm{~m}^{3} / \mathrm{s}$ & $2.438 \mathrm{~m}^{3} / \mathrm{s}$ & $2.977 \mathrm{~m} 3 / \mathrm{s}$ \\
\hline 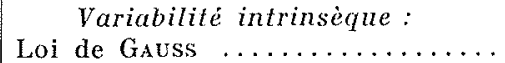 & $Q=2.829(1+=/ 2,45)$ & & $Q=3.557(1+z / 1,93)$ \\
\hline 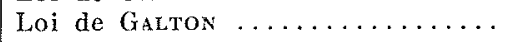 & $\log Q=3,44+0,182 z$ & $\log Q=3,42+0.141 z$ & $\log Q=3,52+0,309 z$ \\
\hline Loi de Gumber $\ldots \ldots \ldots \ldots \ldots \ldots$ & $Q=2.456,2+682 y$ & $Q=2.438+492 y$ & $Q=2.977+1.017 y$ \\
\hline Loi de Fulder $\ldots \ldots \ldots \ldots \ldots \ldots$ & $Q=2.456(1+0,638 \log \mathrm{T})$ & $Q=2.438(1+0,524 \log T)$ & $Q=2.977(1+0,79 \log \mathrm{r})$ \\
\hline Loi de M. Coutagne & $Q=1.201(1+2,31 \sqrt{\log \mathrm{T}})$ & & \\
\hline
\end{tabular}

les plus sèches: $1893,1894,1895,1909,1911,1921,1924$, $1943,1947,1949$, on obtient l'équation:

$$
\frac{\mathrm{Q}}{\mathrm{Q}_{0}}-1=1,31\left(\frac{\mathrm{H}}{\mathrm{H}_{0}}-1\right)+\frac{z}{8,35}
$$

dont le coefficient de corrélation est : $\mathrm{R}=0,79$.

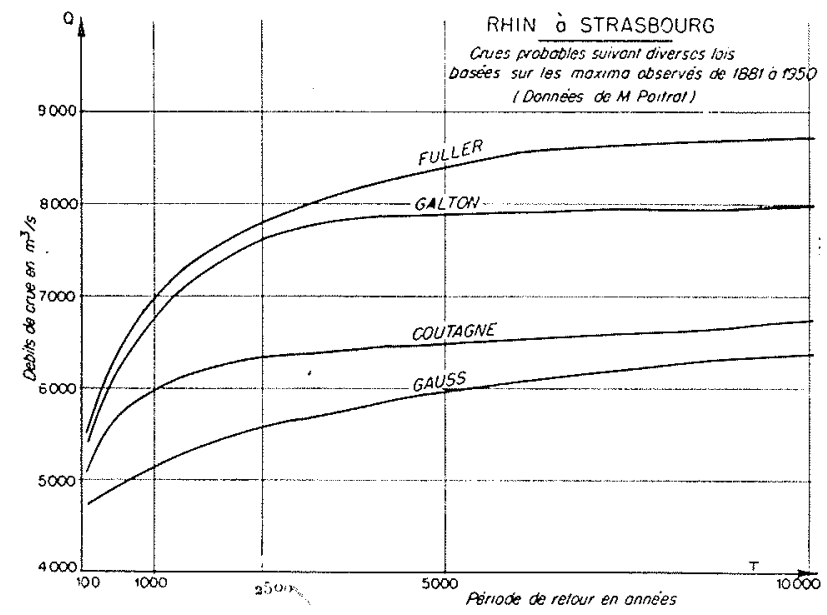

FIG. 5 .
Si, d'autre part, l'on etudie les maxima des mois des huit crues les plus fortes : septembre 1881, septembre 1897, juin 1910, décembre 1918, décembre 1919, mai 1930 ,

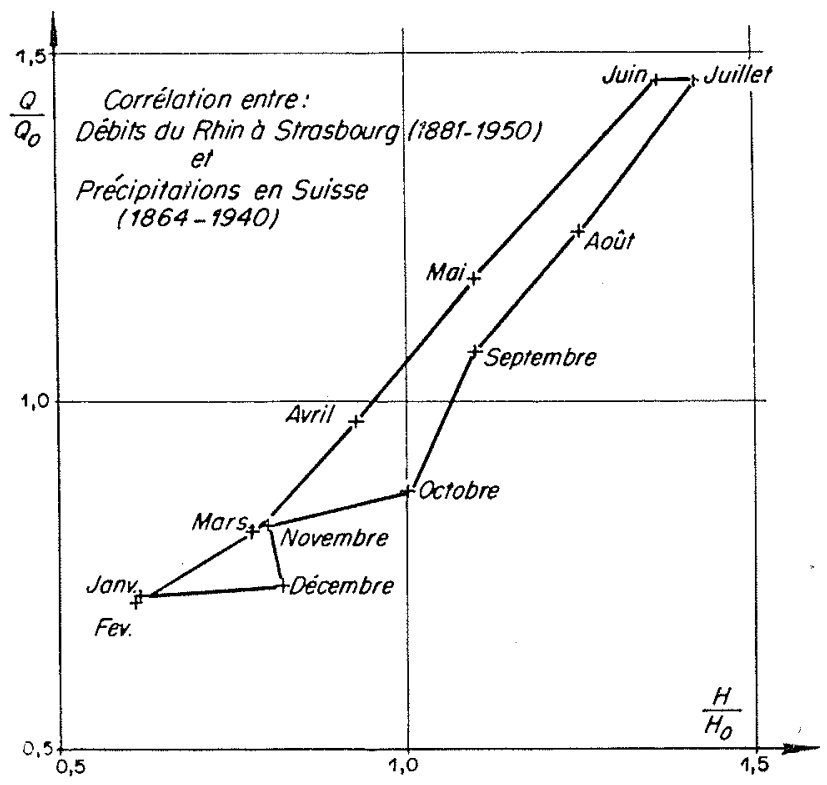

Fig. 6. 
novembre 1944, juillet 1948, la corrélation est exprimée par l'équation :

$$
\frac{\mathrm{Q}}{\mathrm{Q}_{0}}-1=1,1\left(\frac{\mathrm{H}}{\mathrm{H}_{0}}-1\right)+\frac{z}{7,46}
$$

avec $R=0,77$.

Ces corrélations ne sont pas très bonnes mais pourraient sans doute être améliorées par une étude plus approfondie faisant intervenir notamment : une moyenne pondérée des précipitations aux dix stations suisses considérées suivant leur altitude, l'année hydrologique au lieu de l'année civile, la température, la fusion nivale, etc.

\section{Commentaire de $M$. Le Président}

M. le Président remercie $M$. Boyen.

Cette communication a un double intérêt : d'une part, en ce qui concerne le problème du Rhin, et d'autre pari elle rejoint les préoccupations d'ordre général qui ont été analysées dans les communications précédentes et notamment dans celle de M. Moriat.

Vu l'heure tardive, M. Ie Président demande aux membres qui auraient des observations à plésenter de les adresser par note à $\mathrm{M}$. Boyer.

M. le Président remercie les personnes qui ont assisté aux diverses séances et déclare close la session.

La séance est levée à 12 h 30 .

Anticipant sur cette invitation, M. Covtage nous al adressé les graphiques ci-après relatifs aux cycles thermo-pluvial, thermo-fluvial ot pluvio-fluvial (fig. 7) du Rhin à Bâle (année moyenne 1826-1925).

Les précipitations prises en compte sont les mêmes que celles de l'étude de M. Borer. Les écarts thermiques sont déduits des normes de la température a Strasbours de 1891 à 1934. tielle

M. Coutagne signale qu'une interprétation exponen-

$$
\frac{\mathrm{Q}}{\mathrm{Q}_{0}}=e^{\alpha\left(\mathrm{T}-\mathrm{T}_{0}\right)} \quad \text { et }-\mathrm{H}_{\mathrm{H}}=e^{\alpha \prime\left(\mathrm{T}-\mathrm{T}_{0}\right)}
$$

serait plus satisfaisant que la covariation linéaire mentionnée 4 la figure $7(a)$ et donnerait des écarts moindres
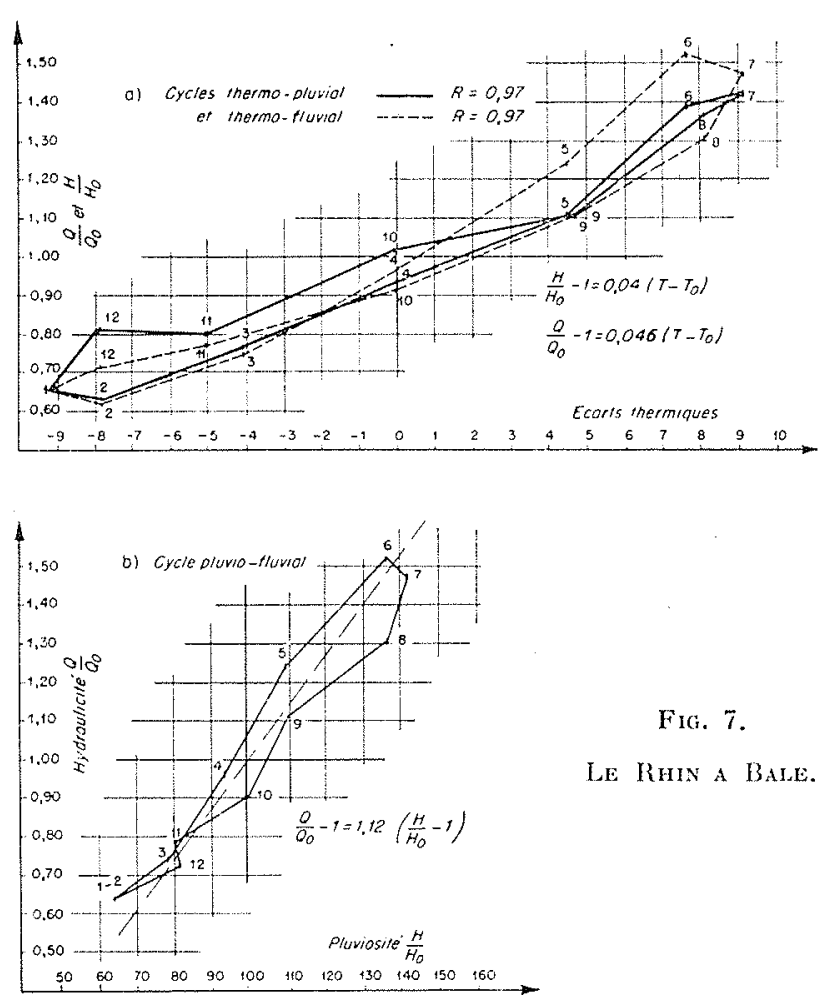

Fig. 7.

LE RHIN A BALE.

Covariations et corrélations de l'année moyenne.

(se reporter à son « Etude générale des covariations de l'année moyenne $\gg$ ).

La comparaison des résultats communiqués par M. Couthane avec ceux de l'étude ci-dessus confirment l'influence de la température sur la corrélation pluviofluviale et l'existence d'une covariation entre les stations de Bâle et de Strasbourg. 


\section{Session des 20, 21 et 22 novembre 1951}

Ont participe aux travaux de cette session:

\section{a) Conseil d'administration :}

MM. Devun, Mary, Miche, Nizery, de Rouville.

MM. Carlier, Legros, Melot représentant respectivement :

MM. Blanc, Coyne, Hupner.

\section{b) Adhérents :}

Electricité de France, représentée par MM. Alacary, Auroy, Birard, Blanchet, Bonnin, Bovvard, Chabert, Combe, Contez, Duranton, Germain, Juprllat, Leblond, Leterrier, Lugiez, Marguenet, Michon, Molbert, Morlat, Monlet, Pannier, Prigent, Ract-Madoux, Ravier, Sudgury, Simon, Trividic, Vantroys.

Electricité et Gaz d'Algérie, représenté par M. Durano. Office National de la Navigation, représenté par M. François BEAU.

Etablissements Neyrpic, représentés par MM. ALExandne et SuQuet.

Compagnie Industrielle de Travaux, représentée par MM. Gosselin et Le Chatelier.

Société des Grands Travaux de Marseille, représentée par MM. Biard, Disereus, Droz, Gondorn, Noullet.

Société Ossude, représentée par M. Bazir.

Société Dauphinoise d'Etudes et de Montages, représentée par M. RABAUd.

Société Hydraulique et Urbanisme, représentée par M. PONSAR.

\section{c) Comité technique :}

M. le Président Barritron.

M. Darrieus, Président de la Commission pour l'étude des $\mathrm{PD}^{2}$.

M. Radiguen, Président des Commissions de Révision des Cahiers des Charges des Conduite Forcées en Métal et en Béton.
M. Bergeron, Vice-Président de la Section «Machines 》. M. Réménínas, Secrétaire général.

MM. Aragou, Banal, Benorst, Biesel, Chamayou, Danel, Dhaille, Dubin, Duffaut, Ferry, Grbert, Gougenhem, Grider, Labaye, Lacombe, Lafteche, Latiras, larrieu, Lefoulon, Lescall, Lomband, de Maublanc, Sanson, Sauvage de Saint-Marc, Serra, Thimel, Toumasse, VaLEMBoIs, VenNin, Wafil.

M. Boyer, Secrétaire.

MM. Gerlier, Lieber, Madin, Turc, représentant respectivement :

MM. Launent, Gaden, de Saint-Vaulry, Hénin.

\section{d) Invités :}

Institut de Mécaniques des Fluides de Lille, représenté par M. Martinot-Lagande.

La Météorologie Nationale, représentée par M. KrveroviTck.

L'Administration des Eaux et Folèts, représentéc par MM. Bouverot, Chmits, Garavel, Labovdigue, de Larre de la Dorie, Reneuve, Widmann.

Le Matériel Electrique S.W., représenté par MM. Gassiot et Manon.

L'Energie des Mers, représentée par M. Christian Beau.

La Compagnie Sulzer, représentée par M. Chareyron.

Mle Garenc, attachée au C.N.R.S.

M. Avrru, Ingénieur des Ponts et Chaussées.

M. LecoQ, du Syndicat Prodisège.

$$
\text { S'étaient excusés : }
$$

M. le Président Gariel.

M. Hurner, Président de la Section «Génie Civil et Conduites » et de la Commission pour l'Etude des Débits de Crue.

MM. Bouvoud, Charron, Coutagne, Crescent, Ehrmann, Escande, Esclangon, Gaden, Kampe de Féniet, Legendre, Mérier, Montagne, Parde, Schlag, Sentenac, Silber, Tricart.

\section{SÉANCE DU MARDI MATIN 20 NOVEMBRE 1951}

La session est ouverte à $9 \mathrm{~h}, 30$, sous la présidence de M. Barillon, Président du Comité Technique.

M. le Président communique les noms des Membres ou personnalités qui se sont excusés de ne pouvoir assister à la session.

\section{Distinction honorifique}

M. Ie Président est heureux de signaler au Comité Technique la promotion au grade de Commandeur de la Légion d'honneur de l'un de ses Membres, M. ParnesTrer, Vice-Président du Conseil général des Ponts et Chaussées. Le Comité Technique s'associe aux félicitations que son Président présentera à M. PArmentrier.

\section{Admissions au Comité Technique}

Le Comité approuve l'admission des personnalités ciaprès, présentées par M. Barillon :

M. Panodr, Membre de l'Institut.

M. Bravdeau, Ingénieur au Service des Etudes et Recherches Hydrauliques d'E.D.F.

M. Durand Robert, Ingénieur au Laboratoire Dauphinois d'Hydraulique.

N. Garier, Paul, Ingénieur aux Etablissements Neyrpic.

M. Halmbons, Ingénieur des Ponts et Chaussées, en service détaché au Laboratoire Dauphinois d'Hydraulique.

M. Juplltar, Ingénieur au Laboratoire National d'Hydraulique. 
M. Micron, Ingénieur au Laboratoire National d'Hydraulique.

M. Monlat, Ingénicur au Service des Etudes et Recherches Hydrauliques d'E.D.F.

\section{Documents reçus depuis la dernière session}

M. le Président signale la réception des documents ciaprès qui viennent cnrichir la bibliothèque de la S.H.F.

L. Bamrabe. - Les Caractères hydrogéologiques de la Guadeloupe.

D. Gaden et L. Borex. - A propos de chambre d'équilibre, infuence de la loi de bariation de la puissance sur la condition de stabilité de Thoma.

H. Lacombe. - Les Marées de la Manche.

A. D. Benhan. - The estimation of extreme flood discharges by statistical methods (L'évaluation des débits maxima de crues par les méthodes statistiques).

A. P. Grant. - Soll conservation in Neab-Zealand (Conservation des sols en Nouvelie-Zélande).

J. C. Schonfend, - Propagation of tides and similar waves (Propagation des marées et des ondes similaires).

Dégäts causés par les inondations et travalur de défense . projetés ou exécutés en Asie el Extrême-Orient (n ${ }^{\circ} 1$, Bangkok, janvier 1951) (2 ex.) (Nations Unies).

Memorandum über die Notlage der gemeindlichen gewerblichen und industriellen Wasserwirtschat im Gebiet der Deutschen Bundesrepublik (Sur la situation alarmante de l'énergie hydraulique pour les besoins généraux, industriels et professionnels dans le bassin de la République fédérale allemande).

Schnee und Lawinen in den Wintern 1936-1937 bis 1945-1946 and 1949-1950 (Neige et avalanches pendant les hivers 1936-1937 a $1945-1946$ et $1949-1950$ (2 volumes). Institut de Weissfluhjoch, Suisse).

River gaugings (Jaugeages de rivières). State of Victoria, Australia). Vol. 1939 : relevés de 1924 à 1936 inclus; vol. 1945 : relevés de 1937 à 1942 inclus.

Les Forces hydrauliques disponibles de la Suisse:

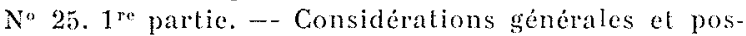
sibilités d'accumulation dans le bassin de l'Aar.

N" 26. - 2" partie. - Bassin du Reuss et du Léman.

$\mathrm{N}^{\circ}$ 27. - 3" partie. - Bassin du Rhin jusqu'au lac de Constance.

$\mathrm{N}^{\circ}$ 28. - $4^{\mathrm{e}}$ partie. - Bassin du Thur, de l'Inn, de l'Adda et du Rom.

No 29. - $5^{\circ}$ partie. - Bassin du Tessin.

Du Bundesanstalt für Gewässerkunde (Service Hydrographique Fédéral de Biclefeld (Allemagne):

Trois annuaires hydrologiques: Bassin superieur du Rhin au-dessus du Main, sans le Neckar (années 19411945). Bassin cotier de la mer du Nord et de la Baltique (année 1947) et Bassin de la Weser (année 1947).

Aménagements hydrauliques entre la mer da Nord el la Baltique, édité par le Service Hydraulique du Ministère du Ravitaillement, de l'Agriculture et des Forêts du Schleswig-Holstein.

Les Voies navigables à l'exposition de construction «Constructa», à Hanovre.

Règles pour le tarage, l'exploitation et l'entretien des limnimètres, limnigraphes et marégraphes, avec 9 annexes (Pegelvorschrift).

Variations des niveaux des eaux souterraines dans le bassin fédéral allemand de février 1950 au printemps 1951 (Grammann Rudolf).

La Pollution des eanx dans les conts d'eau canalisés (Eckold Martin).

Sur la Fidélité des mesures au moulinet (NaTERManN Ernst).
Nouvelles Recherches pour la pêche au jeune saumon pendant l'été (Gensenich Johannes).

Annuatire Hydrologique de la Suisse, 1950.

Bolletino Mensile (Ufficio Idrografico del Magistrato alle Acque. Venise), janvier, février, mars, avril, mai 1950.

Annali Idrologici (Ufficio Idrografico del Magistrato alle Acque. Venise). Année 1947 et année 1948.

J. Sanson. - La Sécheresse remarquable de la période 1941-1949 en France.

- Notes et correspondance. Bilan pluviométrique des 5 annces agricoles 1945 à 1949-1950.

- La Sécheresse et la chalear du printemps et de l'été 1947 et leurs conséquences agricoles.

-- Les Anomalies du climat parisien du XllI au XVIII ${ }^{\circ}$ siécle, d'après les sorties de la chásse de sainte Geneviève.

- L'Eté 1948.

J. LanRas. - Recherches expérimentales sur le défertement des lames.

- La Prébision par le calcul de l'amplitude de la houle en chaque point d̀ l'intérieur ou d̀ l'avant d'un port.

Laboratoire Central d'Hydraulique. - Etude expérimentale de la réflexion de la houle sur des obstacles accores.

S. FenRy et F. Lugrez. - - Prévisions d'upport pendant la période de fusion des réserves nivales.

Ch. Púvy. - Les Premiers Enseignements morphologiques des expéditions polaires francaises, d'après M. Boye, Professeur à la Faculté de Bordeaux

De Quervan. - Die Metamorphose des Schneekristalls (Métamorphose du cristal de neige).

Compte rendu du Comité National Francais de Giodésie et Géphysique (année 1948 et 1949) (par M. Tard).

Imousn. - Prises d'ean frontales el asine-ponts. "Bulletin de l'Union des Ingénieurs Dauphiné-Savoie ». $1^{\text {er }}$ trimestre 1951. (Ce mémoire a été soumis à l'examen de la Section « Génie Civil et Conduites».)

A. Coutagne. - Introduction d̀ l'étude rationnelle de l'hydrologie fluviale (1929).

- Etude analytique des débits de crue (1930).

M. le Président remercic les auteurs de ces ouvages des dons faits à la S.H.F.

\section{Activité de la Société}

M. le Président porte à la connaissance du Comité Technique les travaux des différentes branches de la Société :

La Commission de Répision du Code d'Essais des Installations Hydrauliques, présidée par M. Escande, a mis sous presse les deux premiers fascicules sur la mesure des débits :

"Méthode Chimique et Méthode Gibson.»

«Méthode de jeaugeage par exploration du champ des vitesses. $\gg$

Le tarage des moulinets, qui a été aussi étudié par cette Commission a donné lieu à la rédaction d'un fascicule, qui a été adopté par l'I.S.O. au cours d'une réunion internationale tenue à Paris en septembre 1951, sous les auspices de l'AFNOR, et qui va ctre mis sous presse.

La Commission S.H.F. poursuit ses travaux par lexamen de la methode des deversoirs pour la mesure des débits.

La Commission ponr l'étude de la détermination des débits maxima des crues a prépoir pour un barrage a continué ses travaux sous la présidence de M. Hupner : elle a examiné la méthode analytique les 22 juin et 
5 juillet et le « problème du déluge $»$, avec un exposé de $M$. Halphen le 12 octobre (1).

La «Tournée Glaciologique 1951 \& a eu licu les 24, 25 et 26 juillet 1951 dans les massits d'Oisans et du Pelvoux, sous la présidence de M. Messines bu SounBrEr, Président de la Sous-Section de «Glaciologie * et avec la participation effective de M. le Président Barrillon, M. Jean Bovchayer, Nembre du Conseil d'Administration de la S.H.F. et d'une quarantaine de Membres. La caravane a visité le glacier de la Pilatte en Oisans, le glacier Noir et le glacier Blane dans le Pelvoux, en passant par le col de Temple-Ecrin. Des exposés sur le terrain ont été faits par:

M. Hurn, sur les variations du glacier de la Pilatte.

M. Widmans, sur les variations du glacier Noir et du slacier Blanc.

M. VeYrer, sur la géographie et la géologie du Massil du Pelvoux.

Les participants ont éte reçus au refuge du glacier Blanc par M. Geonoss, Vice-Président du Club Alpin Français.

$$
\text { « }
$$

Dans une prochaine session du Comité Technique, une séance sera consacrée à l'étude du « déficit d'écoulement $\gg:$ des communications seront présentées par Mu. Coutagne, Parde, HÉnin, 'Truc, etc. M. le Président prie les Membres présents intéressés à cette questions de s'inscrire eux-mêmes ou de signaler au Secrétariat d'antres personnalités susceptibles de s'y intéresser.

\section{$2^{\text {es }}$ Journées de l'Hydraulique Grenoble - 25-27 juin 1952}

M. le Président fait part au Comité Technique de l'organisation des $2^{\text {es }}$ «Journées de l'Hydraulique » qui auront lieu à Grenoble les $25-27$ juin 1952 suivant programme inséré d'autre part (2) et précise que le sujet principal d'étude (transport hydraulique et décantation des matériaux solides) a été choisi pour faire connaitre que les réalisations dans cette forme d'application mettent actuellement Ia technique et l'industrie françaises dans une très bonne situation.

\section{Palmarès de la S.H.F.}

M. le Président donne lecture du palmarès de la S.H.F. pour l'année 1951 et fait part des projets de la Société en matière de prix pour l'année 1952 (2).

\section{Publications de la Société}

M. le président rappelle les ouvrages publiés par la Société depuis la dernière session : Mémoires et Travaux (n $\left.{ }^{\circ} 1-1951\right)$, Stations Hydrométriques Francaises $\left(2^{\circ}\right.$ partie, région du Massif Central), Annuaire Hydrologique de la France d'Outre-Mer, publié par l'office de la Recherche Scientifique Outre-Mer en collaboration avec Electricité de France et la S.H.F., grâce à la diligence de M. Nizery.

Les deux fascicules du Code d'Essais des Installations hydrauliques mentionnés plus haut, et l'Annuaire Hydrologique de la France 1950 , sont actuellement sous presse, et, la troisième partie des « Stations Hydrométriques Francaises 》(région des Pyrénées) est en préparation.

(1) Cette Commission, réunie le 23 novembre sous la présidence de $M$. Barillox, remplacant $M$. Hurver, soufrant, adopte des conclusions provisoires qui seront sotmises au Conité Technique à la prochaine session.

(2) Voir page 113 du présent numéro.
COMMUNICATION DE M. DURAND

chef de service

des lrabaux d'aménagements hydro-électriques

à Electricité et Gaz d'Algérie

\section{RÉGLAGE DES DÉBITS DE L'USINE HYDRO-ÉLECTRIQUE DE FOUM-EL-GHERZA}

\section{Résumé}

L'usine hydro-electrique de Foum-el-Gherza, actuelleen construction, est établie an pied d'un barrage-réservoir situé à proximite de Biskra, et destiné à assurer l'irrigation des onsis, situés à l'aval. L'énergic électrique a donc le caractère d'un sous-produit, et le fonctionnement de l'usine doit être asservi à restituer, à chaque instant, le débit demandé par les irrigations.

Cet asservissement doit tenir compte des conditions particulières de l'aménagement: absence de bassin de compensation, grandes variations et creux très faibles do la charge du secteur local isolé, sur lequel débite cette usine, fonctionnement automatique. On devait, en outre, permettre au barragiste, agent des irrigations, d'obtenir le débit demandé sans intervention dans l'usine.

Le dispositif adopté consiste en une vanne levante, fonctionnant à orifice noyé, installée à la sortie de la galerie qui sert de canal de fuite, et en un asservissement classique au niveau de restitution des machines hydrauliques qui équipent l'usine. L'agent des irrigations peut ainsi obtenir le débit demandé simplement en manœuvrant la vanne levante.

Le mémoire analyse le fonctionnement du système. L'étude de la courbe de remou produite dans la galerie de restitution par la vanne levante permet de déterminer la relation qui existe entre la cote de réglage de l'asservissement des machines, la hauteur de levé de la vanne, et le débit admis dans la séghia d'irrigation. La stabilité des asservissements est vérifiée en établissant la condition théorique à satisfaire et en l'appliquant au cas de Foum-cl-Gherza, pour fixer la valeur du décrément nécessaire à une bonne stabilité industrielle.

Il est fait remarquer que les erreurs possibles susceptibies de se produire sur le débit délivré aux irrigations sont inférieures à celle qui découlerait d'une variation aceidentelle de la cote de restitution égale au décrément. Cette remarque permet de prévoir que le débit de l'ensemble sera contrôlé à moins de $5,5 \%$ près, précision très acceptable pour l'alimentation d'un réseau d'irrigation.

Les ordres de grandeur qui découlent des calculs permettent, au moment de la mise en route, d'obtenir un premier réglage approché des organes de commande et de contrôle. Il sera, en outre, effectué des mesures et des essais systématiques, qui permettront de parfaire ce réglage, et d'apprécier la valeur des hypothèses et des approximations du calcul.

COMMUNICATION DE M. LIEBER

Ingénieur en chef

du Burean d'Etudes Alsthom-Charmilles

\section{UNE CENTRALE HYDRO-ÉLECTRIQUE DE GRANDE PUISSANCE ET DE BASSE CHUTE : OTTMARSHEIM, DISPOSITION ORIGINALE ADOPTÉE POUR LES PERTUIS DU BARRAGE ET LEUR COMMANDE 'AUTOMATIQUE}

Le mémoire de M. Lieber et la discussion qui a suivi sa présentation en séance sont publiés pages 151 et suivantes du présent numéro. 


\section{SÉANCE DU MARDI APRĖS-MIDI 20 NOVEMBRE 1951}

La séance est ouverte à 14 h. 15 , sous la présidence de M. Barrillon.

\section{Adoption du procès-verbal de la session de juin 1951}

Le procès-verbal déposé sur le bureau depuis le matin, n’ayant donné licu à aucune observation, est adopté à l'unanimité.

COMMUNICATION PRESENTEE PAR M. DANEL

en l'absence de M. Carapus

\section{ENTRETIEN DES OSCILLATIONS DES EAUX PORTUAIRES SOUS L'ACTION DE LA HAUTE- MER : EXTRAITS DE LA THẼSE SOUTENUE DEVANT' L'UNIVERSITÉ DE GRENOBLE PAR M. MCNOWN, PROFESSEUR A L'UNIVERSITÉ D'IOWA (U.S.A.).}

\section{Résumé}

\section{SEICHES DANS LES PORTS}

Il est intéressant d'étudier théoriquement sur des cas schématiques simples, l'agitation produite dans les ports sous l'action de la houle du large; en particulier il est possible de mettre ainsi en évidence certains aspects essentiels du problème qui échapperaient à l'observation.

Le professeur Mc Nown a étudie l'agitation produite dans des ports à parois entièrement réfléchissantes; les recherches théoriques ont été complétées par des études expérimentales au Laboratoire Dauphinois d'Hydraulique Neyrpic.

Ces études ont donné lieu à une thèse de doctorat ès sciences mathématiques qui a été soutenue devant la Faculté des sciences de Grenoble, en août 1951.

En ce qui concerne les solutions étudiées théoriquement et satisfaisant aux conditions du probleme, l'auteur distingue les « mouvements résonnants» et les «mouvements non résonnants $»$; les premiers sont caractérisés par une valeur de la période de la houle incidente égale à une des périodes propres du port supposé fermé; pour les secondes la période de la houle n'a pas de rapport avec les périodes propres.

Au moyen d'hypothèses vérifiées par l'expérience, l'auteur a étudié les ports circulaires et les ports carrés; dans le premier cas, on se ramène pour les mouvements résonnants, à des problèmes étudiés par divers auteurs en particulier Rayleigh; le profil de la surface est donné par des fonctions de Bessel. Dans certains cas, l'amplitude de l'agitation à l'intérieur du port est beaucoup plus grande qu'à l'entrée, où elle est déjà le double de l'amplitude existant au large. Dans le cas de mouvements non résonnants, l'amplitude se présente sous la forme d'une somme de termes : l'analyse est plus compliquée.

Pour un port carré ou rectangulaire, l'amplitude est donnée par des fonctions sinusoïdales. Elle peut également être bien supérieure à l'amplitude à l'entrée.

Pour vérifier ces théories on a effectué des expériences dans un bassin spécial : un film a été pris qui montre de façon frappante les mouvements à l'intérieur du port. D'une façon générale les mesures, faites très soigneusement et en de nombreux points du port montrent que la théoric est vérifiée avec une approximation excellente.

\section{Discussion}

M. le Président remercie M. Danel de sa très intéressante communication et se demande, d'une part, si les oscillations représentées n'auraient pas pu être obtenues sans générateur de houle, simplement en secouant la plate-forme d'essai et, d'autre-part, ce qui aurait résulté de lobturation progressive de la brèche par un écran élastique.

M. Danel répond que le générateur de houle a permis de voir l'influence de l'ouverture de la passe dans certains cas d'incidence de la houle ne donnant pas lieu a résonance, cas traités dans la thèse de M. Mc Nown mais non représentés dans le film. M. DANEL ajoute que ces essais confirment le calcul dans les cas de résonance et y suppléent dans les cas sans résonance.

M. BIEsel précise la différence existant entre le clapotis d'intensité finie qui se forme en résonance dans le port expérimental par suite de l'excitation due au batteur, et le mouvement d'amplitude théoriquement infini qui se produirait si lon excitait la masse liquide du même port par un mouvement perpendiculaire d'une paroi, par exemple élastique.

M. DE Rouville demande si on a observé des phénomènes analogues dans des ports naturels sensiblement. circulaires.

M. DANEL répond qu'un film tourné dans un petit port du lac Michigan au cours d'une tempête sur ce lac, montre, en effet, dans le port, une agitation double de l'agitation extérieure.

M. DE Rovville remarque qu'en dehors de l'effet de résonance, la pénétration de l'agitation dans un port large par une entrée relativement étroite devrait produire une atténuation de l'agitation extérieure.

M. DANer répond qu'il en est ainsi dans la mesure où l'énergie se dissipe à l'intérieur du port : il faut que toute l'energie qui entre soit dissipée ou ressorte.

M. Valrmbors précise que l'atténuation par obstacles ou épis construits à l'intérieur du port est assez difficile à réaliser efficacement et sûrement car l'effet de ces obstacles varie dans de très larges limites avec la période de l'agitation.

M. Davel convient qu'il s'agit, en effet, plutôt d'un procédé expérimental que d'un remède pratique à l'agitation.

Sur la demande de M. Ie Président, M. Daner promet de communiquer la thèse de $M$. Mc Nows. (Cette thèse a été déposée au Secrétariat de la S.H.F.).

COMMUNICATION DE M. LACOMBE

DIFFRACTION DE LA HOULE PAR UNE BRĖCHE : EXPOSÉ DE LA SOLUTION EXACTE DE M. J. CARR ET DE M. E. STELZRIEDE ET COMPARAISON AVEC LA SOLUTION APPROCHËE DE M. LACOMBE

\section{Résumé}

M. Lacombe expose la solution exacte du problème de la diffraction de la houle par une brèche (1) ${ }^{\star}$, présentée a Washington en juin 1951 par l'un de ses auteurs (J.-H. Carr).

Par l'emploi des coordonnées elliptiques de Mathieu (2,

(*) Les numéros entre parentheses renvoient a la bibliographie en fin d'article. 
3) l'équation de propagation de la houle peut se décomposer en deux équations de Mathreu et les conditions aux limites se présentant dans le problème posé s'expriment alors très simplement. Adaptant à la houle les travaux de Morse et Rubenstein (4), les auteurs américains ont donné la solution exacte du problème ici évoqué. Mais l'expression de cette solution, sous forme de séries de produits de fonctions de Matheu de divers ordres et de diverses espèces, est tellement compliquée qu'une approximation est nécessaire : remplacer certaines fonctions de Matrieu, par le premier terme de leur développement ce qui n'est pas valable à moins de 2 à 3 longueurs d'onde de la brèche; même dans ces conditions, on a da établir des tables détaillées de fonctions de Mathiev et effectuer des calculs à la machine.

De plus la convergence de la série obtenue, très satisfaisante pour les brèches de faible largeur, devient ensuite trop lente à tel point qu'on doit alors recourir. selon les auteurs américains, a la méthode approchée préconisée par Penney et Price (5).

Pour convrir les besoins pratiques les auteurs américains ont présenté pour 4 largeurs de brèches $(1 / 2,1,2$ et 3 longueurs d'onde) et 7 incidences, de 15 en $15^{2}$ de l'incidence normale à l'incidence rasante, les graphiques d'un facteur d'intensité $I$ égal au produit du carré de l'amplitude relative par rapport a l'amplitude de la houle au large par la distance, en longueurs d'onde, du centre de la brèche au point où l'on cherche l'agitation. Ces graphiques illustrent la forte « directivité » de l'agitation dans les cas de brèches larges et sa diminution pour les brèches étroites. Des maxima secondaires d'agitation existent.

Il était évidemment intéressant de comparer ces yaleurs de I à celles que l'on déduit de la formule que nous avons antérieurement présentée ici (6).

La superposition des graphiques en I, faite dans (7), décèle une concordance remarquable pour les grandes largeurs de brèche ( 2 et 3 longueurs d'onde) et les incidences pas trop éloignées de la normale (angle entre les crêtes initiales et la jetée inférieur à $60^{\circ}$ ). Les écarts augmentent pour les brèches moins larges et surtout pour les incidences éloignées de la normale.

M. Lacombe présente des graphiques montrant dans les divers cas l'ampleur des zones où sa formule conduit à une erreur sur l'agitation atteignant $5 \%$ de l'amplitude de la houle au large. Ces zones sont surtout voisines de la jetée « aval ». A noter d'ailleurs que la comparaison faite par les auteurs américains de la solution exacte et des résultats d'essais sur modèle donncs des écarts également très appréciables. Considérant que les circonstances pratiques sont très éloignées des hypothèses thériques faites dans les deux solutions, que les incidences rasantes ne peuvent être examinées par la theorie en raison de l'effet de masque que joue alors, dans la nature, un bras de la jetée, non infiniment mince par rapport à l'autre et que les largeurs de brèche inférieures à une longueur d'onde sont rarement rencontrées, il semble que la formule de M. Lacombe puisse dans la pratique être de quelque utilité.

\section{Bibliographie}

(1) J. H. CanR et M. E. Stelzhiede. - Diffraction of water waves by break-waters. California Institute of Technology -1951 .

(2) E. Matheu - Mémoire sur le mouvement vibratoire d'une membrane elliptique. Jour. des Math. Pures et Appli., 13, 137, 1868 et.Cours de Physique Mathématique, Paris, 1873.

(3) H. W. Me Lachlan. - Theory and applications of Mathieu functions, Oxford, Clarendon Press, $2^{2}$ éd., 1951.
(4) P. M. Monse et P. J. Rubesstein. - The diffraction of waves by ribbons and slits. Phys. Rev., vol. 54, $1^{\text {er }}$ déc. 1938.

(5) W. G. Penne et A. J. Price - Diffraction of sea waves. Directorate of Miscellaneous Weapons, $\mathrm{n}^{\circ}$ 26, Artificial Harbours 3d, 1944.

(6) H. Lacombe. - La diffraction de la houle en incidence oblique. Mémoires et Travaux de la S.H.F. vol. I-1951 et Erratum, vol. II-1951.

(7) H. LACOMBE. - Diffraction de la houle par une brèche. Bulletin d'information du C.O.E.C., IV annéc, février 1952

\section{Discussion}

M. le Président remercie M. Laconbe de son exposé excessivement clair.

M. Larras souligne que le phénomène ne peut être étudié que comme le cas limite d'un ouvrage d'épaisseur finie, les lignes d'épaisseur nulle considérées étant les limites des paraboles ou des hyperboles représentant les jetées de longueur limitée ou semi-indéfinie. Or ces coniques gardent, à la limite, près des extrémités des courbures assez fortes et qui donnent au musoir des formes bien déterminées : si le musoir est circulaire ou parabolique ou elliptique, on a des effets de Mach qui sont assez differents, spécialement pour les grandes obliquités.

M. DaNer confirme que pour des incidences très obliques, la forme du musoir aurait de l'importance mais que cela n'a pas été étudié; de plus la vague qui court le long de la digue deferle au musoir et il y a une dissipation d'énergie, dont il est difficile de tenir compte dans le calcul. Un film montrant ce qui se passe au musoir sera projeté à une prochaine séance.

M. LaRras rappelle que le calcul montre que la vitesse est infinie au musoir, ce qui, physiquement, est impossible; donc il y a un déferlement.

M. Larras émet le vou d'un développement de la théorie orienté vers les cas de largeurs de brèche inférieurs a trois longueurs d'onde, cas qui paraissent les plus courant dans la pratique et qui ne sont pas garantis par lo calcul : les essais poursuivis par M. LARRAS avec le Laboratoire Dauphinois d'Hydraulique tendent à s'assurer de la valeur des résultats théoriques dans cette zone.

M. Mrche rappelle que les essais faits par les Américains sur modèles réduits n’ont guère confirmé les résultats théoriques de la solution « exacte»: le phénomène dépend, en effet, de la forme du musoir, comme l'a indiqué M. Larras et aussi de la pente des talus. Dans ces conditions, une formule simple, comme celle de M. LACombe qui, nonobstant sa simplicité, donne des différences apparemment moindres que celles relevées en Amérique, et qui devrait encore être contrôlée dans les différents cas pratiques, serait très intéressante.

M. DE Rouvirre se demande s'il n'y a pas, dans le cas du musoir très oblique, un effet de réflexion, au moins partielle, qui interfère avec la diffraction principale, et complique ainsi le phénomène.

M. Miche et M. Larras sont de cet avis.

M. LAcombe considère que l'objection très sérieuse, présentée par M. Larras, sur l'inapplicabilité de sa méthode pour les faibles largeurs de brèches et les grandes incidences, ne doit intervenir pour certains ports seulement, que dans les cas rares die tempêtes levant des houles de très grandes longueurs d'onde et, en outre, de grande obliquité; et dans les autres cas, c'est-à-dire, la très grande majorité des cas pratiques, la méthode doit donner une approximation suffisante. 
HOULE LIHTE ET CLAPOTIS LIMITE

$2 a=$ Amplitude de la houle incidente à la profondeur $h$.

$\mathrm{L}=$ Longueur d'onde.

$=-$

$h=$ Profondeur.

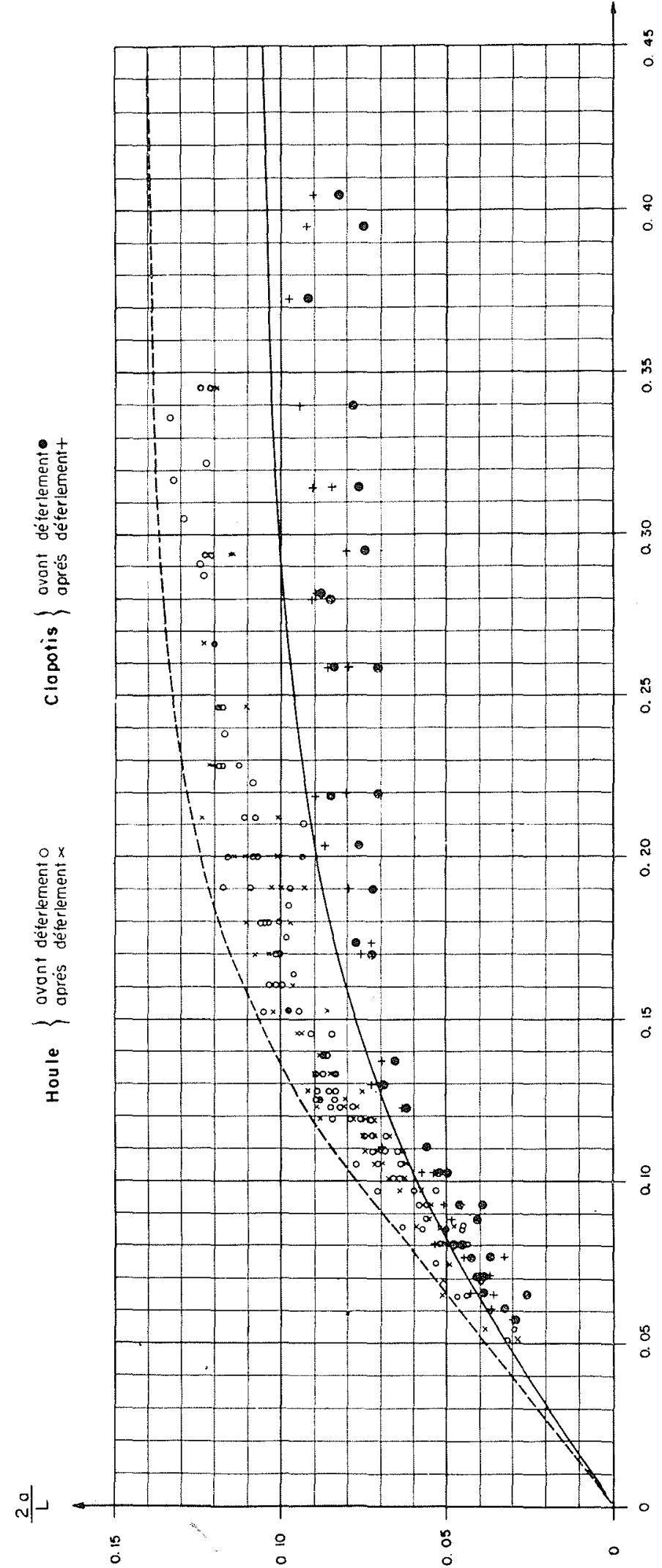

COMMUNICATION DE M. DANEL

\section{Résumé}

\section{HOULE LIMITE ET CLAPOTIS LIMITE}

La houle la plus dangereuse pour un ouvrage ne coïncide pas toujours avec celle dont les dimensions sont les plus importantes car cette dernière ou le clapotis, résultant de la superposition de la houle incidente et de la houle réfléchie, peut déferler en avant de l'ouvrage. Il est donc intéressant d'étudier la houle limite et le clapotis limite.

Cette étude a été faite expérimentalement au Laboratoire Dauphinois d'Hydraulique Neyrpic.

Elle a fait l'objet d'une communication au « Symposium on Gravity Waves » organisé par le National Bureau of Standard à Washington, en 1951, qui a été publiée.

La figure ci-jointe en résume les résultats; sur cette figure on a porté la cambrure de la houle incidente en fonction du rapport profondeur sur longueur d'onde.

M. DANEL commente cette courbe : comme il était en fait impossible de saisir l'instant précis du déferlement, on a serré le phénomène le mieux possible et noté les caracféristiques de la houle immédiatement avant le déferlement et immédiatement après.

En ce qui concerne le clapotis, il projette différentes photographies qui semblent montrer que la surface libre du clapotis présente un point de rebroussement lorsqu'on atteint la forme limite comme le veut une théorie par ailleurs très insuffisante.

Pour la houle, la courbe expérimentale reproduite sur le graphique est assez voisine d'une courbe theorique de M. Miche; au voisinage de l'origine la courbe expérimentale est pratiquement confondue avec la droite theorique que l'on trouve lorsqu'on assimile la houle à une succession d'ondes solitaires. M. DANEr montre plusieurs photographies de houle limite; la crête anguleuse est nette et l'angle des deux versants très voisin de $120^{\circ}$.

\section{Discussion}

M. le Président remercie M. Danel et ouvre la discussion.

M. De Rouvhr.e signale qu'il serait très intéressant d'avoir sur des ouvrages des observations directes de ces phénomènes, et que l'obscrvation très intéressante de M. Danel est que la houle la plus dangereuse est la plus longue de celles qui atteignent directement l'ouvrage. D'autre part, on sait déjà depuis longtemps que la longueur de la houle est un élément très important de la stabilité des talus, mais que l'obliquité de l'ouvrage l'est plus encore. Enfin, il y a peut-être plus de différence, d'une manière absolue, dans les longueurs que dans les hauteurs, entre la houle et le clapotis.

M. Mrchr dégage de cette étude photographique deux résultats intéressants: une vérification nouvelle et précise du fait theorique connu depuis longtemps, savoir que la houle a un angle de $120^{\circ}$ et la preuve expérimentale de l'existence d'un point de rebroussement dans le clapotis limite; ce deuxieme point par contre n'a "pu ètre démontré rigoureusement. parce que le clapotis n'est pas un mouvement pouvant être rendu permanent par un déplacement uniforme des axés, ce qui a permis à Stokes de déterminer l'état limite de la houle.

M. le Président rappelle que la théorie du, clapotis est basée sur la considération de mouvements infiniment petits et de pentes faibles de la surface libre, et cette 
dernière condition n'est pas réalisée au point de rebroussement.

M. Mrche ajoute que l'étude thérique du clapotis limite, nécessiterait l'introduction de la variable temps, ce qu'on peut éviter pour la houle limite.

Repondant à une question de M. Dannevs, M. Mrche indique qu'il existe une présomption théorique en faveur de l'existence du point de rebroussement, dans le clapotis limite, mais que sa valeur est très contestable du rait qu'elle est basce sur une extrapolation de petits mouvements finis : la theorie trochoïdale courante donne cn effet, comme cas limite, une cycloïde elliptique à rebroussement.

Répondant à une question de M. Lannas, M. DaNer précise que la cambrure limite observe dans ces essais est roisine de $0,13-0,14,0,14$ correspondant it la courbe théopique et 0,13 a certains points d'essais en grande profondeur. Malgré les soins apportés la houle expérimentalo n'etait pas completement dépourvue d'harmoniques, aussi a-ton consideré la coube thérique pour les grandes profondeurs et l'onde solitaire pour les très petites profondeurs, et, dans ce dernier cas lacoord était très bon.

En ce qui concerne le déferlement du clapotis d'amplitude finie M. Lannas rappelle que, dans son mémoire publié en 1936 ou 1937 dans les Annales des Ponts ef Chaussées, il a montré la possibilité de déterminer sa forme limite par le calcul en extrapolant la théorie de LEvi-Civita et de STrum; eefle forme serait beancoup plus pointue que celle de la houle, si toutefois elle n'est pas exactement un point de rebroussement.

M. Daner remarque que la houle qui provoque le clapotis est elle-mème assez cambrée.

COMMUNICATION DE M. JACOUES GERMAIN

Ingénieur alu Laboratoire National d'Hydraulique

\section{ÉTUDE DE LA HOULE SUR LES MODÈLES RÉDUITS DE PORTS PAR LA MÉTHODE DU CIEL ÉTOILÉ : APPLICATION AU PORT DE PORT-MARIA DE QUIBERON}

\section{Résumé}

L'étude de l'agitation dans les modèles réduits de ports pose, en général, deux problèmes distinets:

-... Le relevé d'ensemble de ecte agitation dans tout lo modèle,

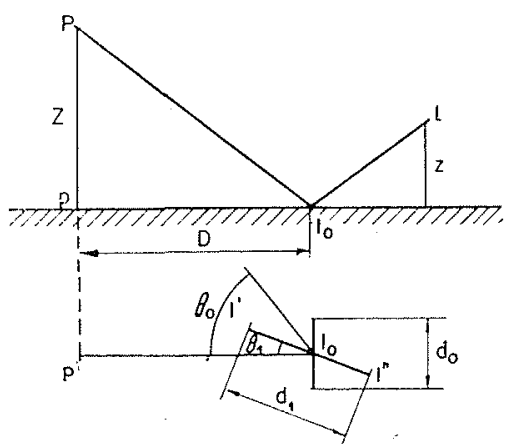

- la détermination, en chaque point, de la nature dn mouvement et de la forme des ondes qui le composent.

La méthode du ciel étoilé permet de résoudre ces deux problèmes avec une excellente précision.

\section{I. - Principe de la méthode}

Le principe de la méthode est connu : il consiste it disposer au-dessus du plan d'eau à chudier un réseau de points lumincux dont on photographic limage dans ce plan d'eau.

A partir de la trajectoire d'une image, on peut déterminer, à chaque instant, la pente du plan tangent à la surface libre au point considéré. Ensuite, a partir de la pente du plan langent, on pourra, moyemant certaines hypothèses sur lat nature de lagitation, déterminer les amplitudes des monvements hopizonlaux et verticaux de l'eau.

L'etude therigue a pour but de lier les parametres qui définissent l'installation $(Z, \approx$, D) a ceux qui définissent la trajectore $\left(d, b_{0}\right.$, a par une relation :

$$
f(z, z, D, d, 0, a)=0
$$

La relation $f$ bant atablie theoriguement dans les differents cas de figure, l'application de la méthode comporte deux opérations :

a) A partir des raleurs maxima de $d$, lues sur la photographie, deduire les valeurs maxima de a (pente da plan tangent a la surface libe);

b) Déduire de ces raleurs maxima de o. l'amplitude verticale, el at besoin horizonlale, du mouvement de l'eau autour de $I_{0}$.

La première operation suppose la comnaissance de lit fonction f ei-dessus, la deuxiene celle de la nature de l'agitation.

\section{II. - Détermination de l'angle " maximum}

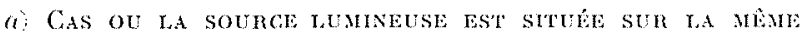

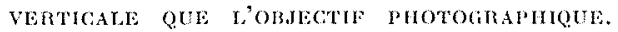

Un raisonnement geometrique simple conduat à la relation :

$$
a=\frac{4 Z z}{Z+z} a
$$

Dans ce cas, une onde de gravité quelconque donne, sur la photographic, un segment de droite.

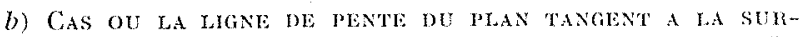

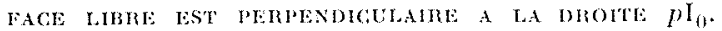

On a, ici encore, la relation :

$$
d=\frac{4 z z}{Z+z} \alpha
$$

c) CAS OU LA LIGNE DE MENTE DU PIAA TANGIST A TA SUR FACE LIBRE WST CONFONDUE RN PROJECTION HORTZONTALE AVEC $p \mathrm{I}_{0}$.

La relation est, dans ce cas:

$$
d=\frac{+Z z}{Z+z}\left(1+\frac{D^{2}}{Z \pm 2}\right) a
$$

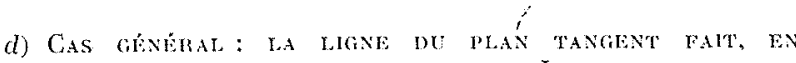
PROJECTION HORIZONTALE, AVEG $p \mathrm{I}_{(B}$, UN ANGLE QULLCONOUE 0 .

Dans ee cas, il y a une distorsion due a l'angle de 
visée: la correction à faire s'exprime par les deux relations :

$$
\begin{aligned}
\operatorname{tg} \theta_{0} & =\left(1+\frac{D^{2}}{Z^{2}}\right) \operatorname{tg} \theta_{1} \\
a_{0} & =a_{1} \frac{\sin \theta_{1}}{\sin \theta_{0}}
\end{aligned}
$$

(indice 0): valeurs exactes; indice 1 : valeurs lues sur la photographie.)

Des abaques tracées une fois pour toules permettent de faire ees corrections instantanément.

\section{III. - Calcul de l'agitation}

L'etude de ce problème releve des théries générales sur chacun des phénomènes étudiés :

Dans le cas d'une houle simple que l'on suppose sinusoïdale, on a, he étant le creux et $A$ la longueur d'onde:

$$
h=\alpha \frac{1}{\pi}
$$

Dans le cas d'une onde solitaire, la formule de Lam donne :

$$
a=\frac{2}{3 \frac{\mathrm{H}}{h} \sqrt{1+\frac{\mathrm{H}}{h}}}
$$

(h: hateur de l'onde; H : tirant d'eau.)
Dans le cas d'une seiche ou d'un clapotis, il y a lieu de passer en revue les diftérents cas qui peuvent so presenter suivant la position de $I_{0}$ pax rapport aux lignes nodales du mouvement.

Dans le cas général, la détermination théorique des relations $h(a)$ ei-dessus est difficile; elle dépend de la forme de profil que lon sera conduit al adopter dans chaque eas.

\section{IV. - Application au port de Port-Maria de Quiberon}

Au cours des études sur le modèle réduit du port de Port-Maria de Quiberon les deux possibilités de la méthode ont èté utilisées.

Divers projets d'aménagement du port avaient été proposés.

Une première séric comprenant 4 dispositions dont chacune devait etre etudié avec 2 niveaux (pleine-mer et hasse-mer) et 8 périodes, ce qui faisait en tout 64 cas différents.

L'emploi systématique de la méthode du ciel étoilé a permis d'explorer rapidement toutes ces dispositions et d'en ehoisir deux entre lesquelles le choix a dû être fait par: une étude quantitative précise des trajectoires.

Des vérifications faites en comparant ces résultats avec des mesures opérées par limnigraphes enregistreurs nous ont montré qu'une précision de l'ordre de $5 \%$ était atteinte dans chaque cas.

La séance est levée à 18 heures.

\section{SÉANCE DU MERCREDI MATIN 21 NOVEMBRE 1951}

La séance est ouverte à 9) h. 15 sous la présidence de M. Miche

\section{COMMUNICATION IIE M. SERRA}

\section{ÉCHO DU CONGRÈS DE L'ASSOCIATION INTERNATIONALE D'HYDROLOGIE SCIENTIFIQUE BRUXELLES, AOUT 1951}

\section{Résumé}

L'Union Géolésique at Géophysique Intermationale a tenu sa IX" Assemblée générale à Bruxelles, du 20 au 30 noût 1951 .

36 nations ayaient envoyé des délégués répartis entro les sept « Associations Internationales » composant T'Union.

Pour la seule Association Internationale d'Hydrologic Seientifique, dont les activités se rapprochent le plus de celles de la Société Hydrotechnicque de France, plus de 150 communications ou rapports, dont 15 français ont clé présentés.

If ne saurait itre question, dans un compie rendu aussi bref, de donner même la nomenclature de ces rapports. Seuls seront signalés ceux qui ont paru prèsenter le plus d'intérêt.

\section{a) Commission des Eaux de Surface.}

M. Svymer (U.S.A.) s'attachant au probleme des crues provenant it la fois des précipitations et de la fonte des neiges, cherche à determiner la part revenant a chacun de ces facteurs.

M. Richaros (Grande-Bretagne) apporte quelques eléments nouvenux sur les procédés d'évaluation des crues.

En ce qui concerne les corrélations pluies-débits et les problèmes relatifs aux prévisions d'apports dans les cours d'eau, il convient de noter les rapports de MM. Covtacine (France), Gorb (Grande-Bretagne), Finny et Lugrez (France), Hoeck (Suisse).

M. SMIETANA (Tchécoslovaquie) adresse des propositions pour la standardisation des earactéristiques hydrologiques a faire figurer dans les monographies des cours d'eau (1).

Le rapport de M. PÉcuy (France) sur la morphométrie n'a pu ètre que résumé à Bruxelles. Quant à celui de M. Serat (France) sur l'interpretation des mesures pluviométriques, il a donné lieu à une discussion animéc dont le résultat a été un projet de création d'un Comité chargé de concilier les divers points de vue en présence.

Au chapitre « Evaporation 》, notons la contribution apportée par M. LANGeren (U.S.A.) sur l'évaporation des surfaces d'eau libres et les résultats intéressants obtenus par M. Wouken (Pays-Bas).

M. DE Quervaln (Suisse) a apporté quelques éléments sur l'evaporation de la converture neigeuse.

Sur l'infiltration, a signaler surtout les rapports de

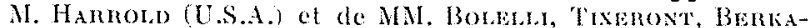
LOFF (France).

(1) L'envoi des propositions de M. Smeraxa avec quelques commentaires sera fait a toutes tes personmes intéressees qui nous en feront la demande. Aples réception des diverses remarcales qui seront fates, l'ensemble pour'a constituer un stjet de communication. 
Erosion des sols et transport des materiaux solides: communications intéressantes de M. BeExch (Canada), Hénen (France) et líaboe (Torvège), ainsi que de M. Braddeau (France) sur les procedés de mesure du debit solide en suspension.

b) Commission des Eacx somtenmatis.

M. Schostrin (France) dresse un état des études regionales des eaux soutertaines en France.

A signaler aussi les communications de Mu. 'Tison (Belgique), Dorre (France), Castaxy (France) qui signale l'établissement de cartes isopiézométriques en funisie.

(c) Commission des Nerges ha Gracks.

M. De Quenval (Suisse) a prescule les propositions d'une Commission crée a Oslo en 1948 pour une classification intemationale de la neige. Le projet a, par la suite, été remis a tous les Membres de la Sous-Section de ciaciologie de la S.H.F. en rue d'etre cramind et discuté.

Notons, au surplus, les intéressantes études de M. Rl-

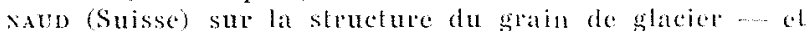

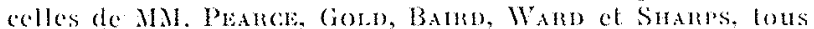

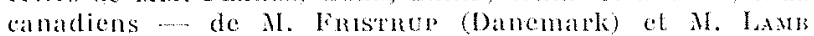
(Giande-Bretasne) sur les observations effectuces sur divers glaciers roisins du Groenland ou du póle Sud.

Grace a l'aetivite de Secretariat général de l'Union Géodésique et Géphysique Internationale, tous les rapports présentes at Bruxelles sont actuellement à l'impression. Nous espérons recevoir dans quelques mois l'ensemble complet de ces études qui constitucnt une contribution très importante aux divers problemes que lon rencontre en hydrologie.

\section{Discussion}

M. Ie Président remereic M. Stema et propose, d'aceord avec M. Bambution, que certaines communications au Congres de bruxelles mentiomées par M. Smra, soient présentees avec phus de détails dans de prochanes reunions du comité Technique, notamment:

1" La communication de M. Srrka lui-meme, sur lá pluviometrie.

-Colle de M. Sxrom, sur les erues provenant a la fois de la fonle des neiges el des precipitations.

-... Celle de M. Smerasa sur la standardisation des caracteres hydrologiques des cours d'ean.

Ces communications seraient présentées par M. Senna: la première en mars 1952, la deuxieme a la Commission du Jébit de elue, la troisième au cours d'une séance ulm lericure.

$2^{\circ}$ Ia communication de M. Protre sur la morphométrie de l'écoulement, qu'il lui sera demandé de présenter luimeme à la session ordinate de mars 1952 .

3" Certaines communications sur les transports de malériaux solides ..... Buevar (Comada), Trson (Bolgique), Krabor (Norvège) - pourraient etre demandes aux ateurs pour les $2^{\text {mi }}$ Journees do l'Hydraulique (juin 1952).

COMMINICATION DE MM. DUFFAUT ET AVRIT

\section{CRUE EXCEPTIONNELLE SUR LA SEUGE (AFFLUENT DE L'ALLIER) LE 16 JUIN 1951}

Ce mémoire et la discussion qui a suivi sa presentation en séance par M. DuFart, sonl publiés pitges 161 et suivantes du présent numéro.
COMMUNICATION DE M. PARDE

\section{PHÉNOMĖNES D'HYDRAULIQUE FLUVIALE OBSERVÉS SUR LE FLEUVE JAUNE \\ PAR L'INGÉNIEUR NORVÉGIEN M. Sig. ELIASSEN, D'APRÉS DES CITATIONS EMPRUNTÉES AUX LETTRES DE CET AUTEUR}

\author{
présentée par M. Borm
}

Résumé

M. Parde, qui a déjà étudié lui-mème le fleuve faune (1) et les transports solides en general (2) transmet of interprete quelques remarques of chiffres intéressants sur la turbidite du fleure Jaune, extrats de sa correspondance avec M. Sig. Erassis, qui a étudie sur place avant la guerre le fleuve Jaune ef le Yang-Tselíang. Il compare les caractères ci-dessus du fleuve Jaune ave ceux de son voisin le Yang-Tsé-Kiang et de quelques autres cours d'eau des diverses parties du monde.

\section{1" Turbidites maxima :}

Fleure Jaune (sables fins de 0,01 ì $1 \mathrm{~mm}$ de diamètre $\ldots \ldots \ldots \ldots \ldots$ ir en poids Colorado ............. _... p 40 San Juan (aflucent du Colorado) - 400

Ririères des Andes seches descendant vers le

Chaco . ............... en poids 60 à 100

Isere moyenne et Durance ..... kg par m: 10

Affluents de l'Isère et de la Du-

rance............. - > > $>100$

Pô, Rhône, Garonne, Rhin (en aval du lac de

Constance) sables grossiers $>1 \mathrm{~mm}$ : quelques kg par m: Yang-Tsé-Jiant . . . . . . . . ' r en poids 5,6 Fleuve Rouge (Indochine) .... … Si-Kiang (Chine meridionale) . _ _ $\quad 0,38$ Nekong . . . . . . . . . 0,5

$2^{\circ}$ Répartilion des turbidités selon les profondeurs.

La valeur relative des turbidites du fleuve Jaune varie de 1 is la surlace it 1,2 on 1,3 it mi-profondeur et 1,4 is 1,6 près du fond; pour le Wei-Ho qui charrie des boues extrêmement fines, la turbidité est la mème sur toute la profondeur, comme dans le sens transversal (ef. Labaye, Note sur le débil solide, Mémoires el tranarax de la S.H.F., n" I-1949, p. (i1).

\section{3" In/luences de la turbidilé:}

a) A partir de $20 \%$ elle accélere la ritesse de rotation des moulinets, par suite du glissement plus important en cau claire des couches liquides sur les ailettes; (mais la turbulenec, qui est généralement plus forte dans les caux claires agit en sens inverse en acélérant de 1 is $2 \%$ les moulinets a axe horizontal dans ces eaux.)

b) Le lapport $\mathrm{Vm} / \mathrm{Vo}$ de la ritesse moyenne a la vilesse superficielle mesuréc au moyen de fotteurs de surface peut decroilte en cau boueuse.

c) Le coefficient de STrickifr $k$ augmente lorsque la turbidité en élements très fins, augmente : il est de l'ordre de 100 pour le Fleuve Janne, de to pour le YangTsé-Kiang et le Mississipi, et de 15 pour les rivières à forte pente et fort charriage de cailloux et de bloes (Pyrénées orientales et Calabre). Cé point parait otre le plus remarquable de l'étude de M. Pande : il rejoint. les

(*) Les chimies entre parentheses renvoient à la bibliographie at la fin de larticle. 
résultats trouvés par Sir Cl. INGLis dans ses recherches sur les canaux des Indes (3).

d) Cet effet accélérateur qui semble dî au nivellement du lit par les dépôts de sable très fin coüncicie, dans les fleaves de plaine à forte turbidité, tels que le fleuve Jaune, avee un affouillement du lit pendant les crues et un remblaiement pendant les décrues (il $y$ a en crue insuiffisance de charge par rapport à la charge limite, donc puissance nette d'érosion; lors des décroissances, le contraire se produit); pour les rivières de montagne très chargée en gros matériaux, l'évolution du lit en crue et décrue est inverse de la précédente, car la charge limite peut y être dépassée de beaucoup par suite de sa valeur relativement réduite à cause de la grosseur des éléments. L'état intermédiaire serait représenté par des cours d'eau apparentés aux deux types extrèmes ci-dessus, tels que le Pô, le Rhone, la Garonne, et le Yang-Tsé-Kiang : sur les seuils en lignes droites, remblaiement en crue, creusements en décrue, et phénomène inverse dans les mouilles (courbes) (4).

\section{Bibliographie}

(1) Parde. - Nouveautés sur le Hoang-Ho (Revue de Géographie de Lyon, vol. XXVI, 1951, n* 1, p. 77, a 97 ).

(2) PARDE. - Sur le Mécanisme des transports solides effectués par les rivières (Revue de Gégraphie Alpine, t. XXXIX, pages 5-40, 289-315 et 758-768).

(3) $\mathrm{Sir}$ Cl. INGLis. - The Effect of variations of charge and grade on slopes and shapes of Channels (A.I.R.H., Grenoble 5, 6, 7 septembre 1949, voir résumé de M. Nizery dans Mémoires et Trabaux de la S.H.F., $\mathrm{n}^{\circ} \mathrm{I}-1950$, page 13$)$.
(4) Parde. - Cours polycopié de dynamique lluviale (Institut Polytechnique, Grenoble, 1942. Epuisé.)

Aunerr. - Cours de l'École Nationale des Ponts et Chaussées.

\section{Discussion}

M. le Président remercie M. Parde et M. Boyen.

V. Banullon s'associe à ces remerciements.

M. le Président croit que le phénomène un peu imprévu de la diminution de rugosité en cas de très forte turbidite pouratit consister non seulement en un nivellement des aspérités du lit, nais surtout en une diminution de la turbulence interne qui améliore l'écoulement lorsque celui-ci est chargé de matières solides fines en suspension. Il y a au contraire, pour le Rhin, dont les graviers sont de l'ordre du centimètre, un accroissement du coefficient de rugosité lorsque le fleuve en crue se met à modifier son fond par charriage. M. Elassex, approuvé de tous points par M. PARDr, croit aussi que la réduction de la turbulence par le transport en masse d'éléments très fin est le principal facteur de diminution pour les coefficients de rugosité. Mais l'effet contraire ne paraît guère douteux et peut-ètre très considérable lorsque lo charriage s'applique à une quantité suffisante de matériaux assez grossiers.

M. Barrillon signale la nécessité de normaliser les expressions telles que turbidité, granulométrie, etc. Les conclusions seraient ainsi beaucoup plus sûres. D'autre part, les nombreux blanes du tableau distribué en séance semblent prourer qu'il y a encore beaucoup à chercher sur la question.

\section{SÉANCE DU MERCREDI APRÈS-MIDI 21 NOVEMBRE 1951}

La sćance est ouverte a 14 h. 30 sous la présidence de iI. Paul Berceron.

COMMUNICATION DE M. BOUVARD

Ingénieur à la R.E.H. Alpes II d'E.D.F.

\section{A PROPOS DE LA CONDITION DE THOMA DANS LES CHEMINÉES A ÉTRANGLEMENT OPTIMUM}

Le mémoire de M. Bouvard est publié pages 168 et suivantes du présent numéro.

COMMENICATION IS M. REMENIERAS

\section{DISPOSITIF SIMPLE POUR RÉDUIRE LA CÉLÉRITÉ DES ONDES ÉLASTIQUES DANS LES CONDUITES EN CHARGE; APPLICATION A LA PROTECTION CONTRE DES COUPS DE BÉLIER}

Le mémoire de M. Remínenas et Ia discussion qui a suivi son exposé en séance sont publiés pages 172 et suivantes du présent numéro.
COMMUNICATION DE M. RAMBAUD

Ingénieur des Recherches et Essais

à la Société Datuphinoise d'Etudes et de Montages

\section{LES POSSIBILITÉS DES TUYAUX AUTO-FRETTÉS SURPRESSÉS ET LEUR UTILISATION A LA CONSTRUCTION DE LA: CONDUITE DE CESTRĖDE}

\section{Résumé}

Lorsqu'on soumet un barreau d'acier à un effort croissant jusqu'au-delat de la valeur correspondant à la limite clastique du metal considéré, la déformation donnée au barreau ne s'annule pas avec l'effort. La conséquence pratique de eette déformation permanente est un relèvement de la limite élastique du métal.

Prenons l'exemple d'un acier au chrome-cuivre ayant subi un allongement de $5 \%$; la limite élastique est augmentée de $45 \%$, la charge de rupture de $20 \%$, l'allongement de rupture diminué de $5 \%$ et la résilience de $15 \%$. Lacier ainsi obtenu possède des caractéristiques mécaniques comprises entre celles des aciers mi-dur et dur et reste parfaitement soudable.

Le relevement de la limite élastique d'un tuyau s'obtient par expansion à froid, au moyen d'une pression intérieure; le tuyau est dit «surpressé ».

La première conduite surpressée a été construite at Santa Luzia (Portugal) en 1939 (hauteur de chute 327 mètres, diamètre $1650 / 1450)$. 
Les avantages des tuyaux surpressés sur les tuyaux soudés sont la réduction des poids et prix et la vérification au cours de la construction du coefficient de sécrrité.

Nous avons construit en 1950 un tuyau expérimental $(\varnothing 1415, e=13$, longueur 6.000$)$ soudé surpressé a $4 \%$ pouvant supporter une pression supérieure de $40 \%$ à celle du même tuyau non surpressé et sur lequel nous avons, en particulier, vérifié la stabilité des caractéristiques mécaniques.

L'intérêt évident de la technique du surpressage nous a amené à l'étendre aux frettes des tuyaux auto-frettés dont la limite élastique peut ètre relevée de $15 \%$ par un allongement de $2 \%$.

Le tuyau expérimental auto-fretlé surpressé que nous avons construit est constitué d'une paroi $(\varnothing 1.454$, $e=13$, longueur 6.000 ) allongé de $4 \%$ et de frettes (section $80 \times 35$ ) allongées de $2 \%$; il peut supporter une pression supérieure de $35 \%$ à celle du tuyau non surpressé de mêmes dimensions.

Une application intéressante des tuyaux auto-frettés surpressés s'est présentéc avec la conduite de Cestrède.
Concue pour fonctionner sous la pression de mapche de $1.061,1$ mètres on a pu lui faire supporter une pression de 1.167,7 mètres en conservant les mèmes matèriaux poids et coefficient de sécurité. Cette augmentation de charge de $10 \%$ au collecteur à $19,2 \%$ en certains points de la conduite a été absorbéc en faisant varier le degré de surpressage jusqu'à $2 \%$ pour les tóles ef $1 \%$ pour Jes frettes.

L'extension aux tuyaux auto-fretlés de la technicue du surpressage marque une nouvelle etape dans la construction des conduites forcées.

\section{承}

M. le President remercic M. Ramberd de sa communication et des résultats qu'il vient de présenter au Comité Technique en faveur des derniers perfectionnements de la construction des conduites.

La communication de M. Rambaub est suivie de quelques projections de cliches montrant les conduites importantes installées en France, pendant les dernières années.

La séance est levée à 17 heures.

\section{SÉANCE DU JEUDI MATIN 22 NOVEMBRE 1951}

Lat séance est ouverte à $9 \mathrm{~h}, 15$ sous la présidence de il. BARRILLON.

COMMUNICATION DE M. CHABERT

Ingénicur au Laboratoire National d'Hydraulique

\section{DE L'UTILISATION DU PHÉNOMÈNE D'INJECTION DANS UN ÉVACUATEUR DE CRUE. APPLICATION AU BARRAGE DE L'IRIL-EMDA.}

Le mémoire de M. Chanen't et la discussion qui a suivi son exposé en séance sont publiés pages 197 et suivantes du présent numéro.

COMMUNICATION DE M. COUTAGNE

\section{DE LA NEIGE QUI TOMBE A L'EAU QUI COULE : ESSAI D'AJUSTEMENT MATHÉMATIQUE D'OBSERVATIONS NIVOMÉTRIQUES COMMUNIQUÉES PAR M. CHURCH, PROFESSEUR A L'UNIVERSITÉ DE NEVADA} (U.S.A.)

présentée par M. Boyer

\section{Résumé}

La présente étude a pour but la prévision des débits nivaux basée sur des corrélations complexes rationnellement déduites de statistiques, extraites pour la plupart (1) des « Transactions American Geophysical Union (1939 a 1944) et mettant en jen non seulement le facteur nival, plus ou moins prépondérant d'ailleurs,

(1) En plus des bassins americains, il est fait mention de deux autres bassins, celui du Drac en France, et celui du lac Malmagen en Suede. Ponr ce dernier, M. Coutacie a fait tat d'un mémoire très détaille du répute hydrologue suedois Ragnar-Melin. mais d'autres factears secondaires perturbant la corrélation simple nivo-pluviale $Q=f(\mathrm{~N})$.

Parmi ces facteurs secondaires, M. Covtagne en distingue 5 et indique les facteurs « témoins » qui permettent d'estimer comparativement les facteurs qui ne peuvent etre chifrés directement par un phénomène communément mesurable, de telle sorte que toute corrélation complexe repose sur tont ou partie des éléments ci-après.

\section{Facteun pringral : neige}

\section{T'énoins :}

Enneigement mesuré au début de lat fusion.... N

Précipitations hivernales ........... H) $\mathrm{N}=$ Débit hivernal du colles d'eall $\ldots \ldots \ldots \ldots \ldots Q_{h}$. $\mathrm{H}-\mathrm{O}_{h}$

\section{Factevis seconiatres}

Pluie de printemps et d'été ............. $h_{\epsilon}$ Rétention liquide. Témoins:

Pluie de l'automne précédent ........... $h_{a}$

Débit pré-hivernal du cours d'eau ........ $q_{a}$ Débit automnal sonrces ............... $q_{s}$ Nivean nappes sonterraines $\ldots \ldots \ldots \ldots \ldots \ldots \varphi \varphi$

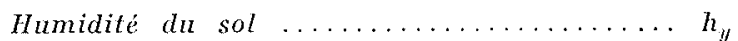
Rétention glaciaire .............. g

Température :

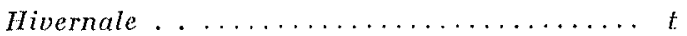

Estivale ...................... $\mathrm{T}$

Débit hivernal $Q_{k}$ tel que $\mathrm{N}=\mathrm{H}-\mathrm{Q}_{k}$

(Pour mémoire, voir: facteurs «témoins» du facteur principal.)

On notera que, parmi les facteur's, les uns interviennent dans la prévision - ceux qui sont connus préalablement à la fusion, et qui sont en italiques dans le tableau ci-dessus - tandis que les autres ne permettent que de la réviser au long de la période de fusion.

Les deux tableaux ci-après (pp. 146-147, 148-149) ré- 
TABLEAU I. CORRELATIONS NIVO-FLUVIALES ET NIVO-PLUVIO-FLUVIALES, par A. Coutagne

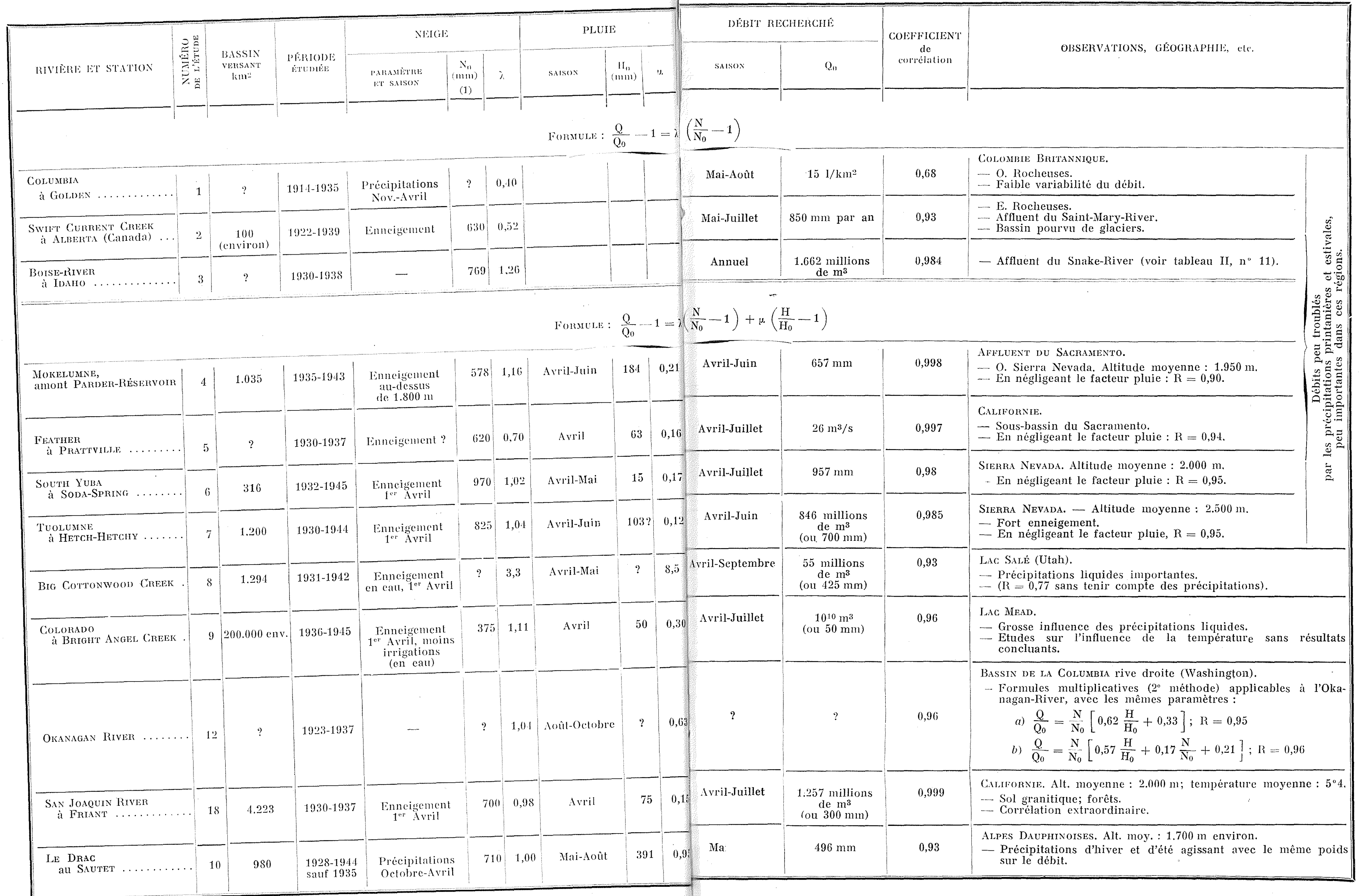

(1) $N_{0}$ chiffre l'équivalent en cau de la couche de neige inestréc directement. 
ThBLEAU 11. - CORRELATIONS Nivoluviales COMPLEXES, par A. Coutagne

ForMULE : $\frac{\mathrm{Q}}{\mathrm{Q}_{0}}-1=\lambda\left(\frac{\mathrm{N}}{\mathrm{N}_{0}}-1+\mu\left(\frac{\mathrm{H}}{\mathrm{H}_{0}}-1\right)+\nu\left(\frac{\mathrm{X}}{\mathrm{X}_{0}}-1\right)\right.$

\begin{tabular}{|c|c|c|c|c|c|c|c|c|c|c|c|c|c|c|c|c|}
\hline \multirow[b]{2}{*}{ RIVIËRE ET STATTON } & \multirow{2}{*}{ 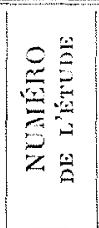 } & \multirow{2}{*}{ 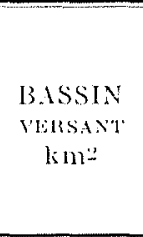 } & \multirow{2}{*}{$\begin{array}{l}\text { PERTIODE } \\
\text { YTLDEEE }\end{array}$} & \multicolumn{3}{|c|}{ NEIGE } & \multicolumn{3}{|l|}{ PLute } & \multicolumn{3}{|c|}{ AUTRES FACTEURS } & \multicolumn{2}{|c|}{ DÉBTT RECHERCHE } & \multirow{2}{*}{ 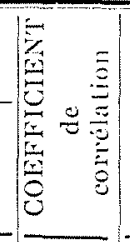 } & \multirow[b]{2}{*}{ OBSLERVATIONS, GEOGRAPHE, etc. } \\
\hline & & & & $\begin{array}{l}\text { ParaMÈthe } \\
\text { ET SAISOS }\end{array}$ & $\left|\begin{array}{c}N_{u} \\
(m m m) \\
(1)\end{array}\right|$ & $\lambda$ & Salson & $\underset{(m m)}{\mathrm{H}_{0}}$ & . & $\begin{array}{l}\text { PARAMÈTRE } \\
\text { ET SAISON }\end{array}$ & $\mathrm{x}_{n}$ & y & SAISON & $Q_{0}$ & & \\
\hline K.IWEAII & 13 & $\begin{array}{c}1.346 \\
-\end{array}$ & $\begin{array}{c}1930-1944 \\
-\end{array}$ & $\begin{array}{c}\text { Enneigement } \\
1^{\text {er Avril }} \\
-\end{array}$ & $\begin{array}{l}817 \\
- \\
\end{array}$ & $\begin{array}{r}1,18 \\
- \\
\end{array}$ & $\begin{array}{r}\text { Avril-Mai } \\
\text { Exclue }\end{array}$ & 120 & 0,1 & $\begin{array}{c}\text { Précipitations } \\
\text { automnales } \\
-\end{array}$ & $120^{\mathrm{mm}}$ & $\begin{array}{c}0,11 \\
0,13 \\
\end{array}$ & Avril-Juillet & $\begin{array}{c}370 \text { millions } \\
\text { de } \mathrm{mm}^{3} \\
\text { oul } 275 \mathrm{~mm}\end{array} \mid$ & $\begin{array}{l}0,995 \\
0,985\end{array}$ & $\begin{array}{l}\text { Sierra Nevada. Lat. } 36^{\circ} \mathrm{N} . \\
\text { - Altitude moyenne }: 1.750 \mathrm{~m} \text {. } \\
\text { - Les pluies deAvril-Mai ne peuvent intervenir dans } \\
\text { les prévisions. }\end{array}$ \\
\hline $\begin{array}{c}\text { Sounw Crmeri } \\
-\end{array}$ & 14 & $\begin{array}{l}123 \\
-\end{array}$ & $\begin{array}{c}1928-1942 \\
-\end{array}$ & - & 448 & 0,40 & & & & $\mathrm{~N}+\mathrm{K}\left(\mathrm{T}-\mathrm{T}_{0}\right)$ & $477^{\mathrm{mm}}$ & 0,45 & $\begin{array}{c}\text { Avril-Sept. } \\
-\end{array}$ & $\begin{array}{c}460 \mathrm{~mm} \\
-\end{array}$ & $\begin{array}{l}0,88 \\
0,95\end{array}$ & 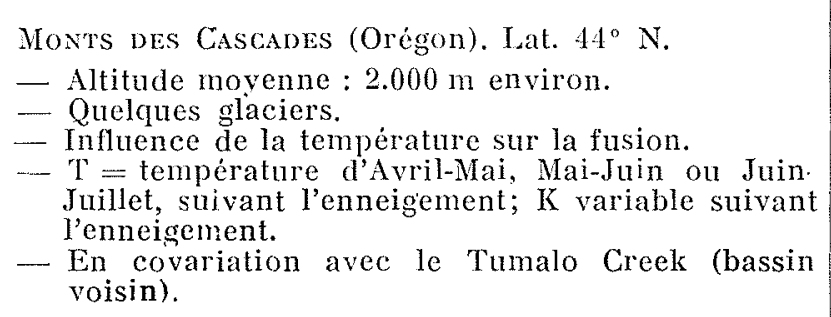 \\
\hline 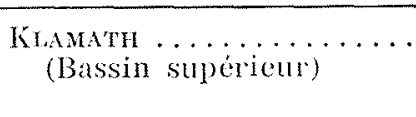 & 15 & 9.000 & $\overline{1925-1942}$ & - & 90 & $\overline{0,40}$ & & & & $\begin{array}{l}\text { Débit des } \\
6 \text { mois } \\
\text { antérieurs }\end{array}$ & $72^{\mathrm{mm}}$ & 1,6 & - & $48 \mathrm{~mm}$ & 0,96 & $\begin{array}{l}\text { OrÉGON, forte capacité de rétention liquide. } \\
\text { - Altitude }: 1.200 \text { a } 1.300 \mathrm{~m} \text {. }\end{array}$ \\
\hline умкана & 16 & $?$ & 20 annèes? & & & & Décembre-Juin & 1.150 & 0,7 & & & & - & $\begin{array}{c}1.500 \text { millions } \\
\mathrm{de} \mathrm{m}^{3}\end{array}$ & $\left|\begin{array}{c}0,94 \\
\text { pour } \\
15 \\
\text { an- } \\
\text { nées } \\
\text { sur } 20\end{array}\right|$ & 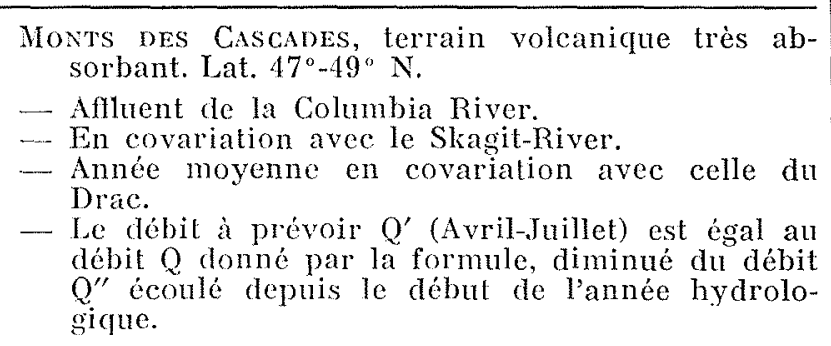 \\
\hline 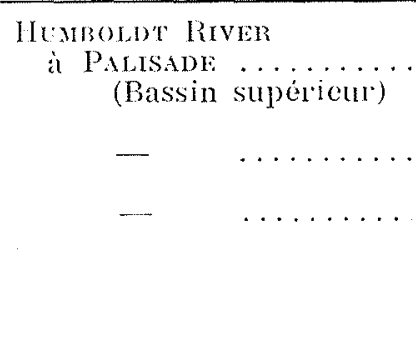 & 19 & $\begin{array}{r}13.000 \\
- \\
-\end{array}$ & $\begin{array}{l}1910-1934 \\
1934-1941 \\
1934-1946\end{array}$ & $\begin{array}{l}\text { Enmeigement } \\
1^{\text {cr }} \text { Mars } \\
- \\
-\end{array}$ & $?$ & $\begin{array}{l}1,60 \\
0,58 \\
0,40\end{array}$ & $\begin{array}{c}- \\
\text { Mars-Juillet } \\
-\end{array}$ & & 0.4 & $\begin{array}{l}\text { Rétention } \\
\text { licuide } \\
\text { (débit } \\
\text { Nov.-Déc. } \\
\text { précédent) }\end{array}$ & & 0,6 & $\begin{array}{l}\text { Mars-Juillet } \\
- \\
-\end{array}$ & $?$ & $\begin{array}{l}0,60 \\
0,96 \\
0,965\end{array}$ & $\begin{array}{l}\text { Nevvad. } \\
\text { - Corrélation très faible (non linéaire). } \\
\text { - Influence de la rétention liquide, facteur essen- } \\
\text { tiel, étant donné la faiblesse des précipitations } \\
\text { annuelles (250 ì } 300 \text { mm). }\end{array}$ \\
\hline 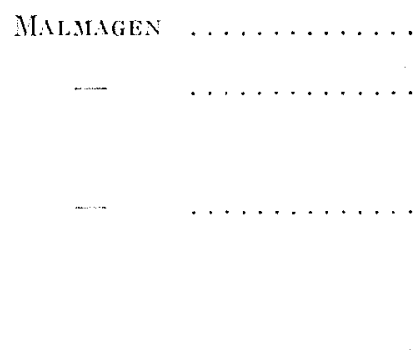 & 17 & $\begin{array}{l}109 \\
-\end{array}$ & $\begin{array}{l}1927-1940 \\
\overline{(1935} \\
\text { excepté) } \\
-\end{array}$ & $\begin{array}{l}\text { Précipitations } \\
\text { moins débits } \\
\text { Octobre-Avril }\end{array}$ & 310 & 0,72 & $\begin{array}{c}\text { Année } \\
\text { Mai-Juin } \\
-\end{array}$ & $\begin{array}{l}900 \\
127\end{array}$ & 1. & $\begin{array}{c}\mathrm{X}=\mathrm{N}+\mathrm{H} \\
\mathrm{N} \text { et } \mathrm{H} \\
\text { ctant définis } \\
\text { comme } \\
\text { ci-dessus }\end{array}$ & $137^{m+1}$ & 1 & $\begin{array}{c}\text { Amnée } \\
\text { Mai-Juin } \\
-\end{array}$ & $\begin{array}{r}753 \mathrm{~mm} \\
437 \mathrm{~mm} \\
437 \mathrm{~mm}\end{array}$ & $\begin{array}{l}0,87 \\
0,95 \\
0,95\end{array}$ & 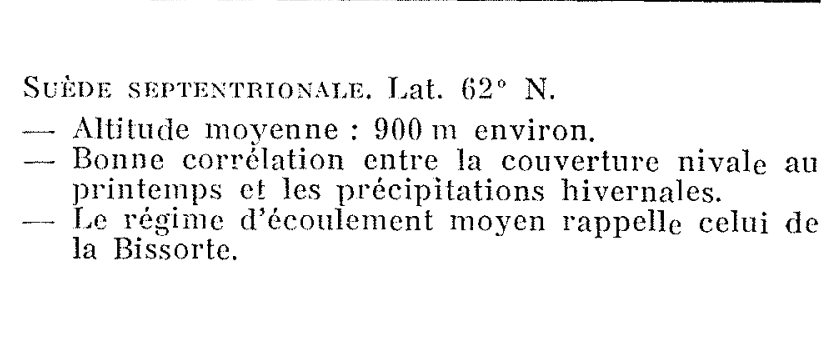 \\
\hline 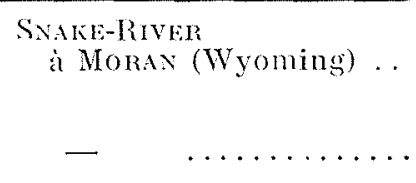 & 11 & $?$ & $\mid \begin{array}{c}1919 \text { à } 1938 \\
\text { - }\end{array}$ & $\begin{array}{l}\text { Enneigement } \\
13 \text { Avril } \\
-\end{array}$ & 580 & $\begin{array}{l}0,96 \\
0,85\end{array}$ & Octobre & 42 & 0,1 & Débit octobre & $16 \mathrm{mun}$ & 0,14 & $\begin{array}{c}18 \text { Mars } \\
30 \text { Septembre } \\
-\end{array}$ & $\begin{array}{c}950 \text { millions } \\
\mathrm{de} \mathrm{m}^{3} \\
--\end{array}$ & $\begin{array}{c}0,92 \\
\leqslant 0,92\end{array}$ & $\begin{array}{l}\text { Rocheruses (Parc National de, Yellowstone). } \\
\text { - Affluent de la Columbia, } \\
\text { - Influence de l'humiditè du sol avant enneige- } \\
\text { ment. }\end{array}$ \\
\hline
\end{tabular}


sument les fésultats obtenus par M. Cotratine pour les dix-neuf rivières étudiées; ils domnent pour chacune d'elles, les caractéristiques principales, les normales des débits et des facteurs qui les conditionnent, el, pour ces derniers, les coeffeients $\lambda$, $u$, vermetlant le reconstituer les formules de corrélations. Enfin, la deruière colonne a droite donne les coefficients de comedation de ces formules.

Le tableau I comporte trois corrélations nivo-lluviales simples et un certain nombre de corrélations nivo-pluviolluviales, à deux variables, le facteur secondaire étant constitue par les précipitations.

Le tableau If concerne des corrélations plus ou moins complexes du débit avec un, deux ou trois factents : neige, précipitations liquides, températures, débit hivernal, ces facteurs pouvant ètre considérés isolément ou combinés.

\section{Discussion}

M. le Président remercie M. Boyen de son exposé al M. Covtagne de la peine qu'il s'est donnée à analyser les travaux américains et à les présenter sous une forme homogène. M. le Président souligne l'intérèt des deux tableaux dressés par $M$. Boyer et qui permettent de voir d'un seul coup d'œil l'ensemble des dix-neuf cas de corrélations.

M. Luciez précise que la colonne \& Rapport de corrélation » devrait, selon la terminologie moderne, s'intituler « coefficient de corrélation 》 et demande lat vérification et l'homogénéisation des valeurs de $\mathrm{N}$ doni certaines sont exprimées en «valeur en eau et d'autres en hauteur de couche de neige.

M. Legrez attire également lattention sul le falt que les valeurs des " coefficients de corrélation » donnes dans les tableaux ne doment une idée exacte de la próvision que pour autant que les correlations mettent en jeu des facteurs connus au moment de lá prévision.

M. Boyer est bien de cet avis of précise que toul ce qui est qualifié d'estival dans le synopsis des facteurs ci-dessus ne peut être utilisé que pour vérifier les prévisions, et non pour les établir.

Enfin. M. Luanz signale que plusieurs de ces coeficients ne peuvent être acceptés qu'avec réserre, parce qu'ils sont calculés sur des périodes extrêmement courtes et peuvent comporter d'importantes erreurs d'echantillonnage.

M. Le Président remercie M. Learez de ses remarques intéressantes dont il sera tenu le plus grand compte pour l'édition des tableaux définitifs.

\section{PRÉSENTATION DU FILM "SNOW-HARVEST" DU U. S. SOIL CONSERVATION SERVICE,}

\author{
par M. Jucilet \\ Ingénicar au. Service de la Production Hydranlique \\ d'Eleclicilé de France
}

Le film fail suite à une demande adressée par la Sous-Section de Glaciologie au doctenl Chunch, de l'Unirersité de Nevada.

Certaines vaes montrent le matériel et les methodes employés aux Etats-Unis pour les mesures d'enneigement. Quelques chiffres concernant le snow-surveying permettront de faire une comparaison avec ce qui se fait en France.

Aux Etats-Unis des mesures analogues a celles qui font l'objet de ce film sont exécutées depuis 1910. Actuellement, dans les principaux bassins de montagne, environ 800 courses sont faites chaque années et 15.000 emplacements de sondage visité. Le personnel mis en couve a la date choisie pour ees mesures, e'est-in-dire aux environs du $1^{\text {or }}$ avril, est d'environ 2.000 personnes comprenant les opéraleurs et les personnes chargées du dépouillement et de la centralisation des mesures.

En France, les premières mesures de ce genre n'ont commencé qu'en 1941 et c'est l'ex-Société des Forces Motrices Bonne et Drac qui en a pris l'initiative. L'expérience s'est étendu peu à peu, mais c'est surtout depuis la nationalisation de l'electricité que les mesures sont faites systématiquement dans les Alpes et les Pyrénées ainsi qu'en quelques endroits du Massif central. Chaque annee, 110 conrses de sondages sont ainsi effectues et 500 emplacements visités. Le nombre d'opérateurs est d'environ 150 (agents F.D.F. suides, ete) et pour le deponillement et la centralisation des mesures, les services d'E.D.F. emploient une dizaine de personnes.

Si l'on tient comple de la surface des zones montagneuses intéressées par les mesures (environ 10 à 12 fois plus importante aux Etats-Unis qu'en France) on constate que l'effectif employe aux mesures est sensiblement le mème aux Etats-Unis qu'en France. Les moyens employés pour la transmission et Ia diffusion des renseignements sont toutefois beaucoup plus modestes chez nous.

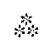

Lat séance est levée à 12 heures après la projection du filn Snow-Harvest.

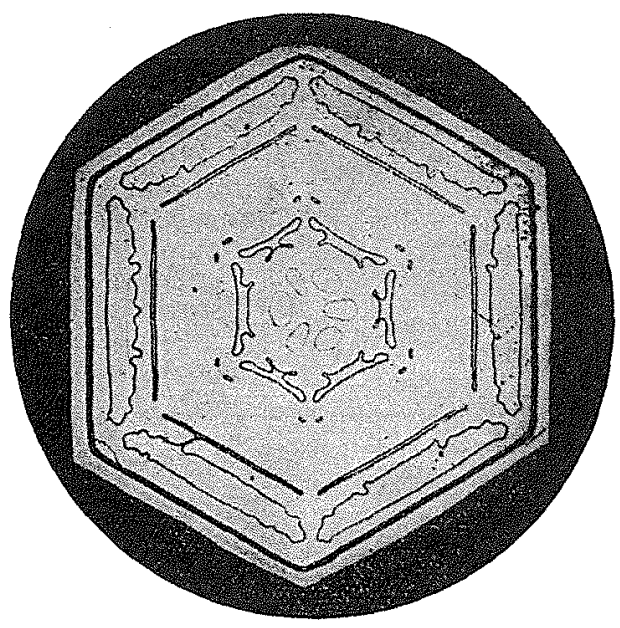

